\title{
Stereochemical control in the reduction of 2-Chromanols
}

Kelin Li and Jon A. Tunge

Supporting information

Table of Contents

General experimental

S-1

Spectral characterization of $\mathbf{2 a} / \mathbf{3 a}$

S-2

Spectral characterization of $\mathbf{2 b / 3 \mathbf { b }}$

S-4

Spectral characterization of $\mathbf{2 c / 3} \mathbf{c}$

S-6

Spectral characterization of $\mathbf{2 d} / \mathbf{3 d}$

S-8

Spectral characterization of $\mathbf{2 e / 3 e}$

S-10

Spectral characterization of $\mathbf{2 f} / \mathbf{3 f}$

$\mathrm{S}-12$

Spectral characterization of $\mathbf{2 g} / \mathbf{3 g}$

S-14

Spectral characterization of $\mathbf{2 h} / \mathbf{3 h}$

S-16

Spectral characterization of $\mathbf{2} \mathbf{i} / \mathbf{3} \mathbf{i}$

S-18

Spectral characterization of $\mathbf{2} \mathbf{j} / \mathbf{3} \mathbf{j}$

$\mathrm{S}-20$

Spectral characterization of $\mathbf{2 k} / \mathbf{3} \mathbf{k}$

$\mathrm{S}-22$

Spectral characterization of $\mathbf{2 l} / \mathbf{3 l}$

$\mathrm{S}-24$

Spectral characterization of $\mathbf{4 a}$

S-26

Spectral characterization of $\mathbf{4 b}$

S-30

Spectral characterization of $\mathbf{4 c}$

S-33

Spectral characterization of $\mathbf{4 d}$

S-36

Spectral characterization of $\mathbf{4 e}$

S-39

Spectral characterization of $\mathbf{4 f}$

S-43

Spectral characterization of $\mathbf{4 g}$

S-46

Spectral characterization of $\mathbf{4 h}$

S-49

Spectral characterization of $\mathbf{4 i}$

S-53

Spectral characterization of $\mathbf{4} \mathbf{j}$

S-56

Spectral characterization of $\mathbf{4 k}$

S-59

Spectral characterization of $\mathbf{4 l}$

S-62

Structure Calculations

S-64

\section{Experimental.}

Materials. Solvents were purged with $\mathrm{N}_{2}$ and passed through two columns of neutral alumina prior to use. Unless otherwise stated, all reagents were used as received. Phenyl lithium was purchased from Acros Organics as a $2 \mathrm{M}$ solution in 70\% cyclohexane-30\% ether. Dihydrocoumarins were readily prepared in good to excellent yield via TFA catalyzed hydroarylation of cinnamic acids according to our literature method. ${ }^{1}$ NMR spectra were referenced to residual proton solvent signals. Structural assignments are based on ${ }^{1} \mathrm{H},{ }^{13} \mathrm{C}$, DEPT-135, and HMQC spectroscopies. Calculations were done using the Gaussian program. ${ }^{2}$

General procedure for the addition of phenyl lithium to dihydrocoumarins.

The dihydrocoumarin $(0.64 \mathrm{mmol})$ was suspended in toluene $(15 \mathrm{~mL})$ and cooled to -78

${ }^{\circ} \mathrm{C}$. Next a solution of $\mathrm{PhLi}$ in butyl ether $(2 \mathrm{M}, 0.58 \mathrm{mmol}, 0.29 \mathrm{~mL}, 0.9$ equiv.) was 
added dropwise via syringe pump over the period of $30 \mathrm{~min}$. The reaction was quenched after $2 \mathrm{~h}$ by adding saturated $\mathrm{NaHCO}_{3}$ at $-78{ }^{\circ} \mathrm{C}$. After extraction of the product with EtOAc, the solvent was evaporated, and the residue purified by column chromatography with EtOAc/hexane.

\section{General procedure for the distereoselective reduction of silanes.}

The ketone/lactol mixture $(0.22 \mathrm{mmol})$ was dissolved in $\mathrm{CH}_{2} \mathrm{Cl}_{2}(2 \mathrm{~mL})$ and cooled to $78{ }^{\circ} \mathrm{C}$. Next, silane $(3.3 \mathrm{mmol})$ was added followed by the addition of $\mathrm{BF}_{3} \cdot \mathrm{Et}_{2} \mathrm{O}(0.88$ $\mathrm{mmol}$ ). After $1 \mathrm{~h}$, the solvent was removed and the residue was purified by column chromatography with $\mathrm{CH}_{2} \mathrm{Cl}_{2} /$ hexane mixtures.

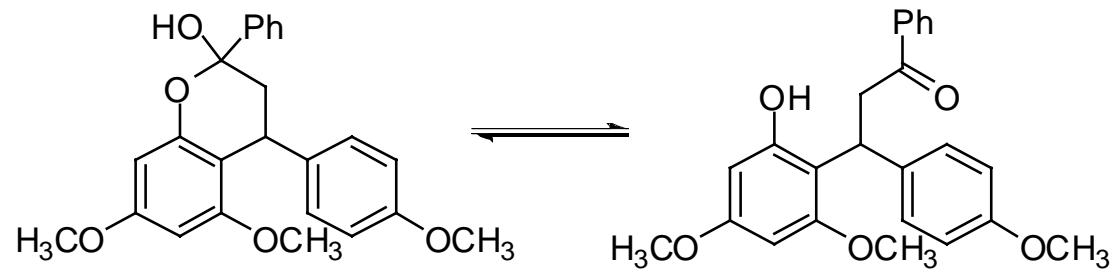

Column chromatography on silica gel $\left(\right.$ EtOAc/hexane $\left.=1 / 4, R_{\mathrm{f}}=0.2\right)$ to afford $\mathbf{2 a} / \mathbf{3 a}$.

$\mathbf{2 a} / \mathbf{3 a}=0.54: 1, \mathbf{2} \mathbf{a} \mathrm{dr}=1: 1$

2a ${ }^{1} \mathrm{H}$ NMR $\left(\mathrm{CDCl}_{3}\right) \delta 7.66(\mathrm{~d}, 2 \mathrm{H}, P h \mathrm{CO}, J=7.3 \mathrm{~Hz}) ; 7.58(\mathrm{~d}, 2 \mathrm{H}, P h \mathrm{CO}, J=7.3 \mathrm{~Hz})$; 7.54 (t, 2H, PhCO, J = 7.3 Hz); 7.33-7.37 (m, 1H, PhCO); 7.30-7.33 (m, 1H, PhCO); $7.12\left(\mathrm{~d}, 2 \mathrm{H}, P h \mathrm{OCH}_{3}, J=8.8 \mathrm{~Hz}\right) ; 7.03\left(\mathrm{~d}, 2 \mathrm{H}, P h \mathrm{OCH}_{3}, J=8.8 \mathrm{~Hz}\right) ; 6.84(\mathrm{~d}, 2 \mathrm{H}$, $\left.P h \mathrm{OCH}_{3}, J=8.8 \mathrm{~Hz}\right) ; 6.74\left(\mathrm{~d}, 2 \mathrm{H}, P h \mathrm{OCH}_{3}, J=8.8 \mathrm{~Hz}\right) ; 6.31\left(\mathrm{~d}, 1 \mathrm{H}, P h \mathrm{OCH}_{3}, J=2.0\right.$ $\mathrm{Hz}) ; 6.24\left(\mathrm{~d}, 1 \mathrm{H}, P h \mathrm{OCH}_{3}, J=2.0 \mathrm{~Hz}\right) ; 6.18\left(\mathrm{~d}, 1 \mathrm{H}, P h \mathrm{OCH}_{3}, J=2.0 \mathrm{~Hz}\right) ; 6.09(\mathrm{~d}, 1 \mathrm{H}$, $\left.P_{h \mathrm{OCH}_{3}}, J=2.0 \mathrm{~Hz}\right) ; 4.30-4.33\left(\mathrm{~m}, 1 \mathrm{H}, \mathrm{CHCH}_{2}\right) ; 4.27-4.30\left(\mathrm{~m}, 1 \mathrm{H}, \mathrm{CHCH}_{2}\right) ; 3.82(\mathrm{~s}$, $\left.3 \mathrm{H}, \mathrm{OCH}_{3}\right) ; 3.77\left(\mathrm{~s}, 3 \mathrm{H}, \mathrm{OCH}_{3}\right) ; 3.60\left(\mathrm{~s}, 3 \mathrm{H}, \mathrm{OCH}_{3}\right) ; 3.45(\mathrm{~s}, \mathrm{br}, 1 \mathrm{H}, \mathrm{OH}) ; 3.41$ (s, br, $1 \mathrm{H}, \mathrm{OH}) ; 3.37\left(\mathrm{~s}, 3 \mathrm{H}, \mathrm{OCH}_{3}\right) ; 2.56-2.60\left(\mathrm{~m}, 1 \mathrm{H}, \mathrm{CHCH}_{2}\right) ; 2.43-2.54\left(\mathrm{~m}, 1 \mathrm{H}, \mathrm{CHCH}_{2}\right)$; 1.88-2.05 (m, $\left.1 \mathrm{H}, \mathrm{CHCH}_{2}\right)$.

3a ${ }^{1} \mathrm{H}$ NMR $\left(\mathrm{CDCl}_{3}\right) \delta 8.01(\mathrm{~d}, 2 \mathrm{H}, P h \mathrm{CO}, J=7.3 \mathrm{~Hz}) ; 7.43(\mathrm{t}, 2 \mathrm{H}, P h \mathrm{CO}, J=7.3 \mathrm{~Hz})$; $7.25\left(\mathrm{~d}, 2 \mathrm{H}, P h \mathrm{OCH}_{3}, J=8.8 \mathrm{~Hz}\right) ; 6.80\left(\mathrm{~d}, 2 \mathrm{H}, P h \mathrm{OCH}_{3}, J=8.8 \mathrm{~Hz}\right) ; 6.12(\mathrm{~d}, 1 \mathrm{H}$, $\left.P_{h O C H}, J=2.0 \mathrm{~Hz}\right) ; 5.99\left(\mathrm{~d}, 1 \mathrm{H}, \mathrm{PhOCH}_{3}, J=2.0 \mathrm{~Hz}\right) ; 5.10\left(\mathrm{dd}, 1 \mathrm{H}, \mathrm{CHCH}_{2}, J=9.4\right.$ and $4.4 \mathrm{~Hz}) ; 4.12\left(\mathrm{dd}, 1 \mathrm{H}, \mathrm{CHCH}_{2}, J=17.8\right.$ and $\left.9.4 \mathrm{~Hz}\right) ; 3.73-3.79\left(\mathrm{~m}, 1 \mathrm{H}, \mathrm{CHCH}_{2}\right)$; $3.75\left(\mathrm{~s}, 3 \mathrm{H}, \mathrm{OCH}_{3}\right) ; 3.67\left(\mathrm{~s}, 3 \mathrm{H}, \mathrm{OCH}_{3}\right) ; 3.53\left(\mathrm{~s}, 3 \mathrm{H}, \mathrm{OCH}_{3}\right)$.

Combined ${ }^{13} \mathrm{C}\left\{{ }^{1} \mathrm{H}\right\}$ NMR $\delta 201.2(\mathrm{C}=\mathrm{O}), 159.6$ (Quat.), 159.1 (Quat.), 159.0 (Quat.), 158.7 (Quat.), 157.9 (Quat.), 157.4 (Quat.), 157.2 (Quat.), 155.5 (Quat.), 154.5 (Quat.), 154.3 (Quat.), 143.7 (Quat.), 143.6 (Quat.), 138.7 (Quat.), 136.6 (Quat.), 136.0 (Quat.), 135.5 (Quat.), 133.3 (Arom. CH), 128.5 (Arom. CH), 128.4 (Arom. CH), 128.21 (Arom. $\mathrm{CH}$ ), 128.17 (Arom. CH), 128.14 (Arom. CH), 128.07 (Arom. $\mathrm{CH}$ ), 127.6 (Arom. $\mathrm{CH}$ ), 125.4 (Arom. $\mathrm{CH}$ ), 125.1 (Arom. $\mathrm{CH}$ ), 113.9 (Arom. $\mathrm{CH}$ ), 113.2 (Arom. $\mathrm{CH}$ ), 113.1 (Arom. CH), 112.2 (Arom. CH), 106.9 (Arom. CH), 103.5 (Arom. CH), 98.4 (COH), 97.5 $(\mathrm{COH}), 97.1$ (Arom. $\mathrm{CH}$ ) 95.0 (Arom. $\mathrm{CH}$ ), 94.4 (Arom. $\mathrm{CH}$ ), 93.9 (Arom. $\mathrm{CH}$ ), 93.2 (Arom. CH), 92.5 (Arom. $\mathrm{CH}$ ), 92.2 (Arom. $\mathrm{CH}), 55.4\left(\mathrm{OCH}_{3}\right), 55.3\left(\mathrm{OCH}_{3}\right), 55.24$ $\left(\mathrm{OCH}_{3}\right), 55.20\left(\mathrm{OCH}_{3}\right), 55.15\left(\mathrm{OCH}_{3}\right), 55.06\left(\mathrm{OCH}_{3}\right), 55.03\left(\mathrm{OCH}_{3}\right), 55.0\left(\mathrm{OCH}_{3}\right) 45.3$ $\left(\mathrm{CHCH}_{2}\right), 41.9\left(\mathrm{CHCH}_{2}\right), 41.5\left(\mathrm{CHCH}_{2}\right), 35.2\left(\mathrm{CHCH}_{2}\right), 33.8\left(\mathrm{CHCH}_{2}\right), 32.6\left(\mathrm{CHCH}_{2}\right)$. $V_{\max }(\mathrm{NaCl}) / \mathrm{cm}^{-1}: 3572,3348,3032,3005,1672,1614,1589,1510,1485,1245,1209$, 1201, 1149, 1095, 1035. HR-MS: $\mathrm{C}_{24} \mathrm{H}_{25} \mathrm{O}_{5}$ Calcd. $393.1702(\mathrm{M}+\mathrm{H})$; found: 393.1695. 


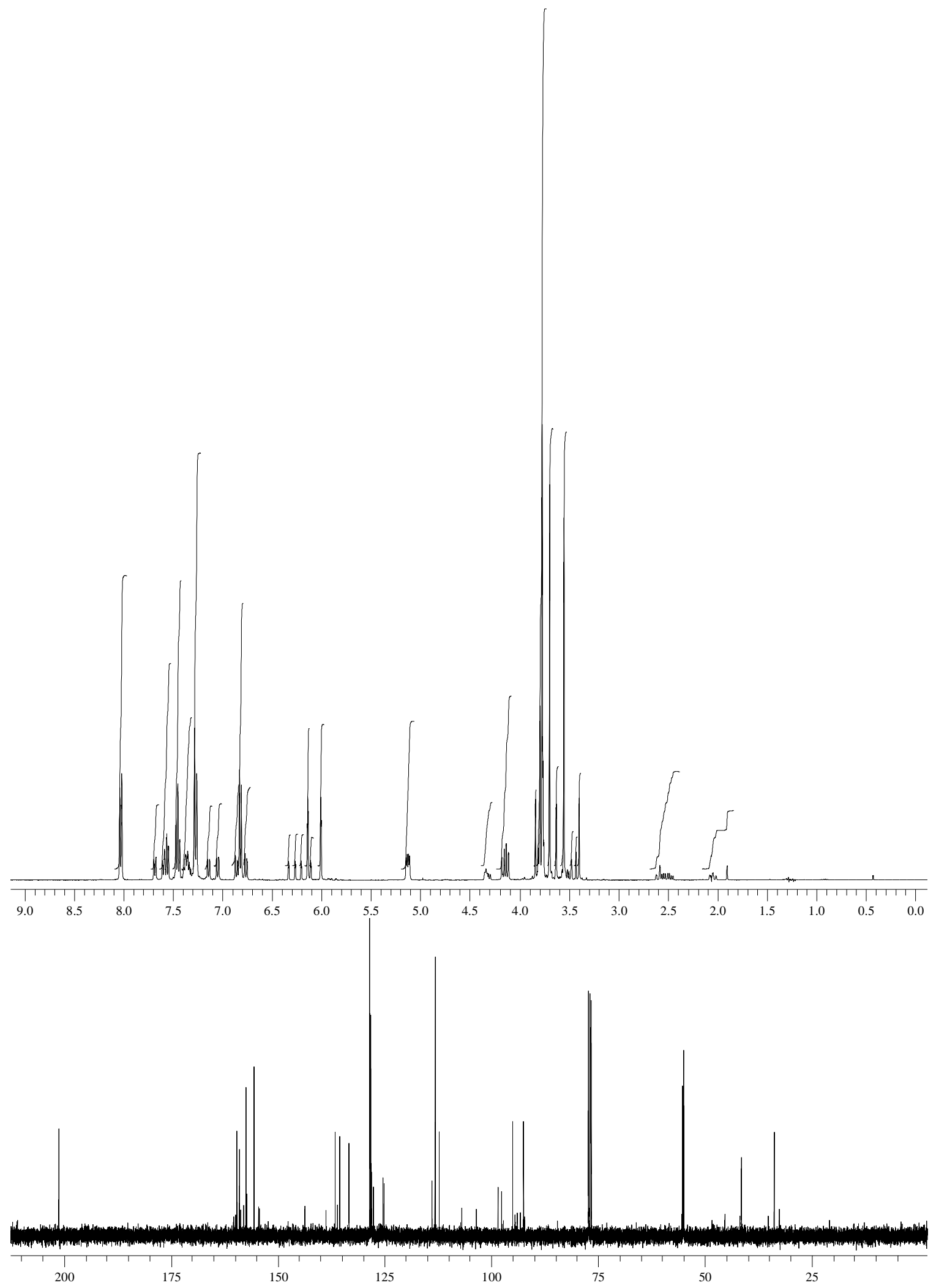

S-3 


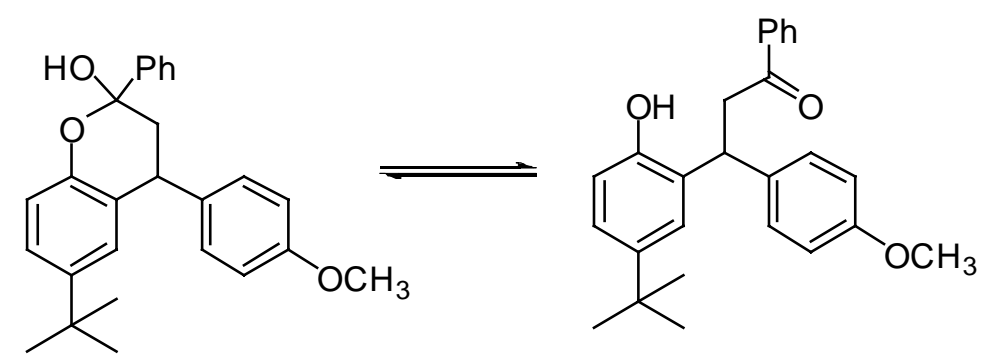

Column chromatography on silica gel (DCM, $\left.\mathrm{R}_{\mathrm{f}}=0.4\right)$ to afford $\mathbf{2 b} / \mathbf{3} \mathbf{b}$.

$\mathbf{2 b} / \mathbf{3} \mathbf{b}=0.69: 1, \mathbf{2} \mathbf{b} \mathrm{dr}=9.6: 1$

2b (major) ${ }^{1} \mathrm{H} \mathrm{NMR}\left(\mathrm{CDCl}_{3}\right) \delta 7.73(\mathrm{~d}, 2 \mathrm{H}, P h \mathrm{CO}, J=7.0 \mathrm{~Hz}) ; 7.58(\mathrm{t}, 2 \mathrm{H}, P h \mathrm{CO}, J=$ $7.0 \mathrm{~Hz}) ; 7.37(\mathrm{t}, 1 \mathrm{H}, P h \mathrm{CO}, J=7.0 \mathrm{~Hz}) ; 7.21\left(\mathrm{~d}, 2 \mathrm{H}, P h \mathrm{OCH}_{3}, J=8.6 \mathrm{~Hz}\right) ; 7.10(\mathrm{~d}, 1 \mathrm{H}$, $\left.P h^{\mathrm{t}} \mathrm{Bu}, J=9.0 \mathrm{~Hz}\right) ; 6.97\left(\mathrm{~d}, 1 \mathrm{H}, P h^{\mathrm{t}} \mathrm{Bu}, J=9.0 \mathrm{~Hz}\right) ; 6.90\left(\mathrm{~s}, 1 \mathrm{H}, P h^{\mathrm{t}} \mathrm{Bu}\right) ; 6.89(\mathrm{~d}, 2 \mathrm{H}$, $\left.P h \mathrm{OCH}_{3}, J=8.6 \mathrm{~Hz}\right) ; 4.50\left(\mathrm{dd}, 1 \mathrm{H}, \mathrm{CHCH}_{2}, J=13.1\right.$ and $\left.5.6 \mathrm{~Hz}\right) ; 3.31(\mathrm{~d}, 1 \mathrm{H}, \mathrm{OH}, J=$ $2.3 \mathrm{~Hz}) ; 2.46\left(\mathrm{dd}, 1 \mathrm{H}, \mathrm{CHCH}_{2}, J=13.6\right.$ and $\left.5.6 \mathrm{~Hz}\right) ; 2.10\left(\mathrm{dd}, 1 \mathrm{H}, \mathrm{CHCH}_{2}, J=13.4\right.$ and $2.3 \mathrm{~Hz}) ; 1.22\left(\mathrm{~s}, 9 \mathrm{H}, \mathrm{Ph}^{\mathrm{t}} \mathrm{Bu}\right)$.

2b (minor) ${ }^{1} \mathrm{H}$ NMR $\left(\mathrm{CDCl}_{3}\right) \delta 3.45(\mathrm{~s}, 1 \mathrm{H}, \mathrm{OH}) ; 2.66\left(\mathrm{dd}, 1 \mathrm{H}, \mathrm{CHCH}_{2}, J=13.4\right.$ and 5.8 $\mathrm{Hz}) ; 2.54$ (dd, $1 \mathrm{H}, \mathrm{CHCH}_{2}, J=13.6$ and $\left.8.8 \mathrm{~Hz}\right) ; 1.23\left(\mathrm{~s}, 9 \mathrm{H}, \mathrm{Ph}^{\mathrm{t}} B u\right)$.

3b ${ }^{1} \mathrm{H}$ NMR $\left(\mathrm{CDCl}_{3}\right) \delta 8.02(\mathrm{~d}, 2 \mathrm{H}, P h \mathrm{CO}, J=7.0 \mathrm{~Hz}) ; 7.46(\mathrm{t}, 2 \mathrm{H}, P h \mathrm{CO}, J=7.0 \mathrm{~Hz})$; $7.42(\mathrm{t}, 1 \mathrm{H}, P h \mathrm{CO}, J=7.0 \mathrm{~Hz}) ; 7.28\left(\mathrm{~d}, 2 \mathrm{H}, P h \mathrm{OCH}_{3}, J=8.6 \mathrm{~Hz}\right) ; 7.09\left(\mathrm{~d}, 1 \mathrm{H}, P h^{t} \mathrm{Bu}, J\right.$ $=9.0 \mathrm{~Hz}) ; 6.87\left(\mathrm{~d}, 2 \mathrm{H}, P h \mathrm{OCH}_{3}, J=8.6 \mathrm{~Hz}\right) ; 6.80\left(\mathrm{~d}, 1 \mathrm{H}, \mathrm{Ph}^{\mathrm{t}} B u, J=9.0 \mathrm{~Hz}\right) ; 6.70(\mathrm{~s}$, $\left.1 \mathrm{H}, P h^{\mathrm{t}} \mathrm{Bu}\right) ; 5.04\left(\mathrm{dd}, 1 \mathrm{H}, \mathrm{CHCH}_{2}, J=8.3\right.$ and $\left.5.3 \mathrm{~Hz}\right) ; 3.90\left(\mathrm{dd}, 1 \mathrm{H}, \mathrm{CHCH}_{2}, J=17.4\right.$ and $8.6 \mathrm{~Hz}) ; 3.83\left(\mathrm{~s}, 3 \mathrm{H}, \mathrm{OCH}_{3}\right) ; 3.79\left(\mathrm{~s}, 3 \mathrm{H}, \mathrm{OCH}_{3}\right) ; 3.78\left(\mathrm{dd}, 1 \mathrm{H}, \mathrm{CHCH}_{2}, J=17.4\right.$ and $5.6 \mathrm{~Hz}) ; 1.21\left(\mathrm{~s}, 9 \mathrm{H}, \mathrm{Ph}^{\mathrm{t}} \mathrm{Bu}\right.$ ).

Combined ${ }^{13} \mathrm{C}\left\{{ }^{1} \mathrm{H}\right\}$ NMR $\delta 200.0(\mathrm{C}=\mathrm{O}), 158.2$ (Quat.), 157.9 (Quat.), 151.1 (Quat.), 150.2 (Quat.), 144.2 (Quat.), 143.6 (Quat.), 143.4 (Quat.), 136.6 (Quat.), 135.7 (Quat.), 135.6 (Quat.), 133.4 (Quat.), 130.4 (Quat.), 129.8 (Arom. CH), 128.9 (Arom. CH), 128.6 (Arom. CH), 128.4 (Arom. $\mathrm{CH}$ ), 128.3 (Arom. $\mathrm{CH}$ ), 128.2 (Arom. $\mathrm{CH}$ ), 126.2 (Arom. $\mathrm{CH}$ ), 125.4 (Arom. CH), 125.2 (Arom. CH), 125.0 (Arom. CH), 124.5 (Arom. $\mathrm{CH}$ ), 124.3 (Arom. CH), 116.6 (Arom. CH), 116.4 (Arom. CH), 113.9 (Arom. CH), 113.8 (Arom. $\mathrm{CH}), 97.5(\mathrm{COH}), 55.2\left(\mathrm{OCH}_{3}\right), 55.1\left(\mathrm{OCH}_{3}\right), 44.7\left(\mathrm{CHCH}_{2}\right), 43.6\left(\mathrm{CHCH}_{2}\right), 38.4$

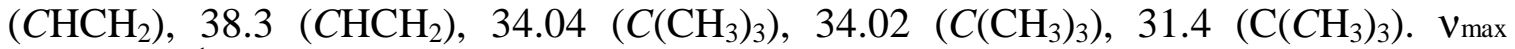
$(\mathrm{NaCl}) / \mathrm{cm}^{-1}: 3569,3034,3005,1676,1610,1581,1512,1496,1363,1247,1180,1034$. HR-MS: $\mathrm{C}_{26} \mathrm{H}_{32} \mathrm{O}_{3} \mathrm{~N}$ Calcd. $406.2382\left(\mathrm{M}+\mathrm{NH}_{4}\right)$; found: 406.2364 . 


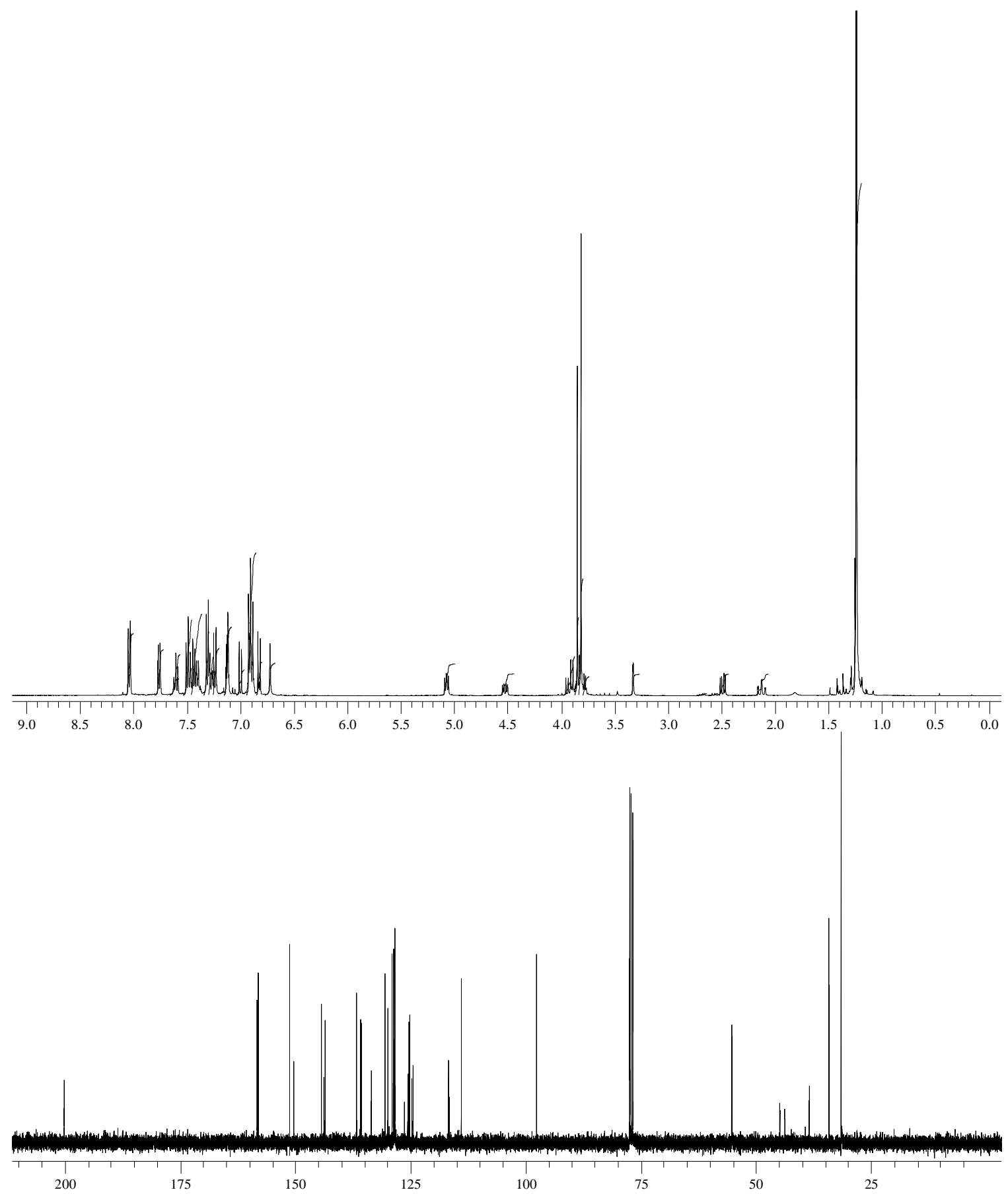

S-5 


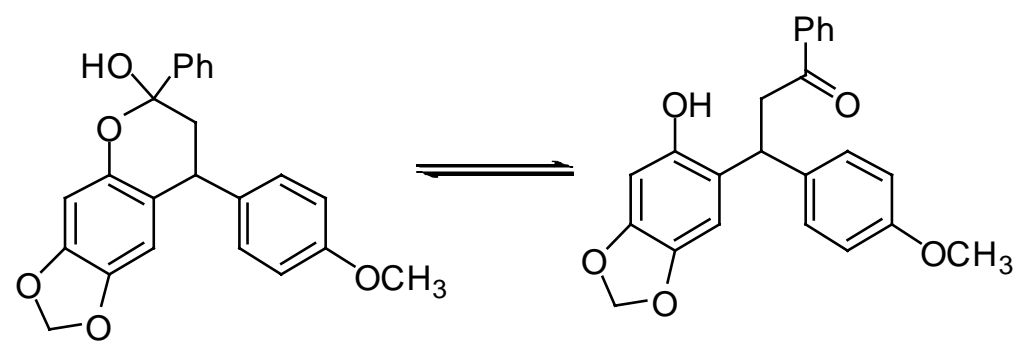

Column chromatography on silica gel $\left(\mathrm{DCM}, \mathrm{R}_{\mathrm{f}}=0.2\right)$ to afford $\mathbf{2 c} / \mathbf{3 c}$.

$\mathbf{2} \mathbf{c} / \mathbf{3} \mathbf{c}=0.64: 1, \mathbf{2} \mathbf{c} \mathrm{dr}=7.8: 1$

2c (major) ${ }^{1} \mathrm{H}$ NMR $\left(\mathrm{CDCl}_{3}\right) \delta 7.69$ (d, 2H, $P h \mathrm{CO}, J=7.3 \mathrm{~Hz}$ ); 7.59 (t, $2 \mathrm{H}, P h \mathrm{CO}, J=$ $7.0 \mathrm{~Hz}) ; 7.37(\mathrm{t}, 1 \mathrm{H}, P h \mathrm{CO}, J=7.3 \mathrm{~Hz}) ; 7.16\left(\mathrm{~d}, 2 \mathrm{H}, P h \mathrm{OCH}_{3}, J=8.6 \mathrm{~Hz}\right) ; 6.86(\mathrm{~d}, 2 \mathrm{H}$, $\left.P h \mathrm{OCH}_{3}, J=8.6 \mathrm{~Hz}\right) ; 6.53\left(\mathrm{~s}, 1 \mathrm{H}, P h \mathrm{OCH}_{2} \mathrm{O}\right) ; 6.27\left(\mathrm{~s}, 1 \mathrm{H}, P h \mathrm{OCH}_{2} \mathrm{O}\right) ; 5.86(\mathrm{~s}, 1 \mathrm{H}$, $\left.\mathrm{OCH}_{2} \mathrm{O}\right) ; 5.82\left(\mathrm{~s}, 1 \mathrm{H}, \mathrm{OCH}_{2} \mathrm{O}\right) ; 4.34\left(\mathrm{dd}, 1 \mathrm{H}, \mathrm{CHCH}_{2}, J=12.9\right.$ and $\left.5.6 \mathrm{~Hz}\right) ; 3.80(\mathrm{~s}, 3 \mathrm{H}$, $\left.\mathrm{OCH}_{3}\right) ; 3.34(\mathrm{~d}, 1 \mathrm{H}, \mathrm{OH}, J=2.0 \mathrm{~Hz}) ; 2.42\left(\mathrm{dd}, 1 \mathrm{H}, \mathrm{CHCH}_{2}, J=13.6\right.$ and $\left.5.8 \mathrm{~Hz}\right) ; 2.03$ (dd, $1 \mathrm{H}, \mathrm{CHCH}_{2}, J=13.4$ and $2.3 \mathrm{~Hz}$ ).

2c (minor) ${ }^{1} \mathrm{H}$ NMR $\left(\mathrm{CDCl}_{3}\right) \delta 7.08\left(\mathrm{~d}, 2 \mathrm{H}, P h \mathrm{OCH}_{3}, J=8.6 \mathrm{~Hz}\right) ; 6.60(\mathrm{~s}, 1 \mathrm{H}$, $\left.P h \mathrm{OCH}_{2} \mathrm{O}\right) ; 6.18\left(\mathrm{~s}, 1 \mathrm{H}, P \mathrm{OCH}_{2} \mathrm{O}\right) ; 3.53(\mathrm{~s}, 1 \mathrm{H}, \mathrm{OH}) ; 2.63\left(\mathrm{dd}, 1 \mathrm{H}, \mathrm{CHCH}_{2}, J=13.6\right.$ and $5.8 \mathrm{~Hz})$.

$3 c^{1} \mathrm{H}$ NMR $\left(\mathrm{CDCl}_{3}\right) \delta 8.01(\mathrm{~d}, 2 \mathrm{H}, P h \mathrm{CO}, J=7.3 \mathrm{~Hz}) ; 7.46(\mathrm{t}, 2 \mathrm{H}, P h \mathrm{CO}, J=7.3 \mathrm{~Hz})$; 7.41 (t, $1 \mathrm{H}, P h \mathrm{CO}, J=7.3 \mathrm{~Hz}) ; 7.25\left(\mathrm{~d}, 2 \mathrm{H}, P h \mathrm{OCH}_{3}, J=8.6 \mathrm{~Hz}\right) ; 6.87\left(\mathrm{~d}, 2 \mathrm{H}, P h \mathrm{OCH}_{3}\right.$, $J=8.6 \mathrm{~Hz}) ; 6.49\left(\mathrm{~s}, 1 \mathrm{H}, P h \mathrm{OCH}_{2} \mathrm{O}\right) ; 6.43\left(\mathrm{~s}, 1 \mathrm{H}, P h \mathrm{OCH}_{2} \mathrm{O}\right) ; 5.78\left(\mathrm{~s}, 2 \mathrm{H}, \mathrm{OCH}_{2} \mathrm{O}\right) ; 4.96$ (dd, $1 \mathrm{H}, \mathrm{CHCH}_{2}, J=10.1$ and $\left.3.5 \mathrm{~Hz}\right) ; 3.86\left(\mathrm{dd}, 1 \mathrm{H}, \mathrm{CHCH}_{2}, J=17.9\right.$ and $\left.10.1 \mathrm{~Hz}\right)$; $3.79\left(\mathrm{~s}, 3 \mathrm{H}, \mathrm{OCH}_{3}\right) ; 3.71\left(\mathrm{dd}, 1 \mathrm{H}, \mathrm{CHCH}_{2}, J=18.2\right.$ and $\left.3.8 \mathrm{~Hz}\right)$.

Combined ${ }^{13} \mathrm{C}\left\{{ }^{1} \mathrm{H}\right\}$ NMR $\delta 200.5(\mathrm{C}=\mathrm{O}), 158.3$ (Quat.), 158.0 (Quat.), 148.0 (Quat.), 146.9 (Quat.), 146.7 (Quat.), 146.2 (Quat.), 143.9 (Quat.), 141.8 (Quat.), 136.2 (Quat.), 135.8 (Quat.), 133.7 (Arom. CH), 129.6 (Arom. CH), 128.7 (Arom. CH), 128.6 (Arom. $\mathrm{CH}$ ), 128.4 (Arom. $\mathrm{CH}$ ), 128.31 (Arom. $\mathrm{CH}$ ), 128.28 (Arom. $\mathrm{CH}$ ), 125.2 (Arom. $\mathrm{CH}$ ), 124.0 (Quat.), 117.8 (Quat.), 113.99 (Arom. CH), 113.96 (Arom. CH), 108.1 (Arom. $\mathrm{CH}), 107.3$ (Arom. $\mathrm{CH}), 100.82\left(\mathrm{OCH}_{2} \mathrm{O}\right), 100.81\left(\mathrm{OCH}_{2} \mathrm{O}\right), 100.0$ (Arom. $\left.\mathrm{CH}\right), 98.8$ (Arom. $\mathrm{CH}), 97.4(\mathrm{COH}), 55.19\left(\mathrm{CH}_{3}\right), 55.18\left(\mathrm{CH}_{3}\right), 44.9\left(\mathrm{CHCH}_{2}\right), 43.3\left(\mathrm{CHCH}_{2}\right), 38.3$ $\left(\mathrm{CHCH}_{2}\right), 36.6\left(\mathrm{CHCH}_{2}\right) . v_{\max }(\mathrm{NaCl}) / \mathrm{cm}^{-1}: 3568,3325,3032,3007,1672,1629,1610$, 1597, 1581, 1512, 1502, 1479, 1248, 1203, 1178, 1149, 1039. 


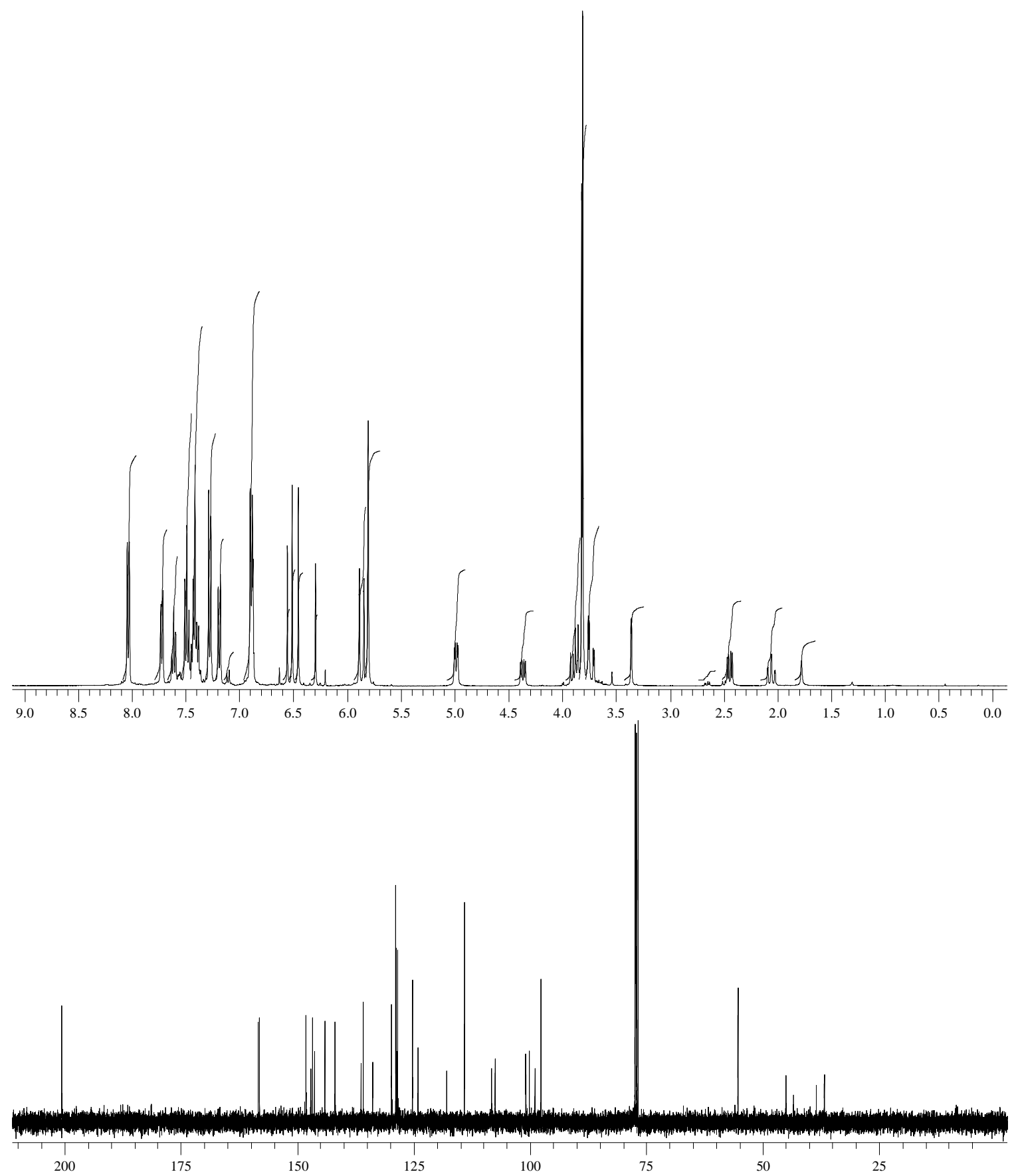




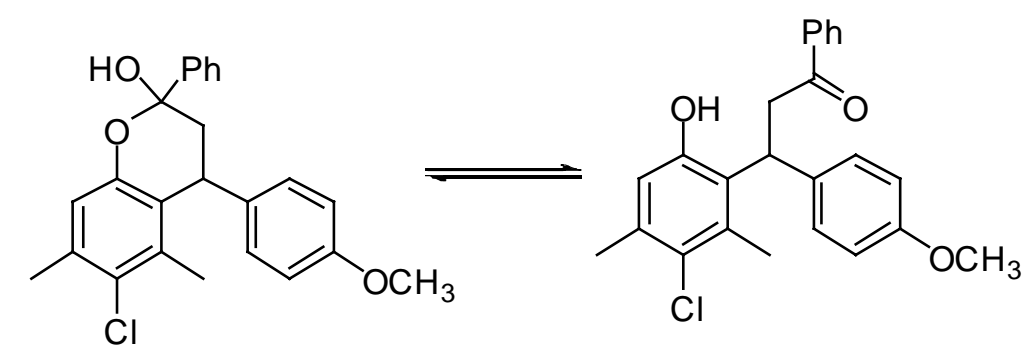

Column chromatography on silica gel $\left(\right.$ EtOAc/hexane $\left.=1 / 4, R_{f}=0.3\right)$ to afford $\mathbf{2 d} / \mathbf{3 d}$.

$\mathbf{2 d} / \mathbf{3 d}=6.4: 1, \mathbf{2 d} \mathrm{dr}=1.7: 1$

2d (major) ${ }^{1} \mathrm{H}$ NMR $\left(\mathrm{CDCl}_{3}\right) \delta 7.65$ (d, 2H, PhCO, $\left.J=7.3 \mathrm{~Hz}\right) ; 7.35$ (t, $2 \mathrm{H}, P h \mathrm{CO}, J=$ $7.3 \mathrm{~Hz}) ; 7.32-7.39(\mathrm{~m}, 1 \mathrm{H}, P h \mathrm{CO}) ; 6.97\left(\mathrm{~d}, 2 \mathrm{H}, P h \mathrm{OCH}_{3}, J=8.7 \mathrm{~Hz}\right) ; 6.83(\mathrm{~s}, 1 \mathrm{H}$, $\left.P h \mathrm{CH}_{3}\right) ; 6.77\left(\mathrm{~d}, 2 \mathrm{H}, P h \mathrm{OCH}_{3}, J=8.7 \mathrm{~Hz}\right) ; 4.42\left(\mathrm{dd}, 1 \mathrm{H}, \mathrm{CHCH}_{2}, J=10.9\right.$ and $\left.7.3 \mathrm{~Hz}\right)$; $3.76\left(\mathrm{~s}, 3 \mathrm{H}, \mathrm{OCH}_{3}\right) ; 3.14(\mathrm{~s}, 1 \mathrm{H}, \mathrm{OH}, J=2.0 \mathrm{~Hz}) ; 2.62\left(\mathrm{dd}, 1 \mathrm{H}, \mathrm{CHCH}_{2}, J=14.1\right.$ and 7.6 $\mathrm{Hz}) ; 2.38$ (s, $\left.3 \mathrm{H}, \mathrm{PhCH}_{3}\right) ; 2.02-2.09$ (m, $\left.1 \mathrm{H}, \mathrm{CHCH}_{2}\right) ; 1.92$ (s, 3H, $\left.\mathrm{PhCH}_{3}\right)$.

2d (minor) ${ }^{1} \mathrm{H}$ NMR $\left(\mathrm{CDCl}_{3}\right) \delta 7.58(\mathrm{~d}, 2 \mathrm{H}, P h \mathrm{CO}, J=7.3 \mathrm{~Hz}) ; 7.32-7.39(\mathrm{~m}, 2 \mathrm{H}$, $P h \mathrm{CO}) ; 7.32-7.39$ (m, $1 \mathrm{H}, P h \mathrm{CO}) ; 7.10\left(\mathrm{~d}, 2 \mathrm{H}, P h \mathrm{OCH}_{3}, J=8.7 \mathrm{~Hz}\right) ; 6.92(\mathrm{~s}, 1 \mathrm{H}$, $\left.P h \mathrm{CH}_{3}\right) ; 6.86\left(\mathrm{~d}, 2 \mathrm{H}, P h \mathrm{OCH}_{3}, J=8.7 \mathrm{~Hz}\right) ; 4.30\left(\mathrm{~d}, \mathrm{br}, 1 \mathrm{H}, \mathrm{CHCH}_{2}, J=5.3 \mathrm{~Hz}\right) ; 3.80(\mathrm{~s}$, $\left.3 \mathrm{H}, \mathrm{OCH}_{3}\right) ; 3.18(\mathrm{~s}, 1 \mathrm{H}, \mathrm{OH}) ; 2.62-2.66\left(\mathrm{~m}, 1 \mathrm{H}, \mathrm{CHCH}_{2}\right) ; 2.47\left(\mathrm{dd}, 1 \mathrm{H}, \mathrm{CHCH}_{2}, J=\right.$ 14.4 and $6.8 \mathrm{~Hz}) ; 2.43$ (s, $\left.3 \mathrm{H}, \mathrm{PhCH}_{3}\right) ; 2.07$ (s, $\left.3 \mathrm{H}, \mathrm{PhCH}_{3}\right)$.

3d ${ }^{1} \mathrm{H}$ NMR $\left(\mathrm{CDCl}_{3}\right) \delta 8.00(\mathrm{~d}, 2 \mathrm{H}, P h \mathrm{CO}, J=7.3 \mathrm{~Hz}) ; 7.46(\mathrm{t}, 2 \mathrm{H}, P h \mathrm{CO}, J=7.3 \mathrm{~Hz})$; $7.15\left(\mathrm{~d}, 2 \mathrm{H}, P_{h \mathrm{OCH}_{3}}, J=8.7 \mathrm{~Hz}\right) ; 6.59\left(\mathrm{~s}, 1 \mathrm{H}, P h \mathrm{CH}_{3}\right) ; 5.25\left(\mathrm{t}, \mathrm{br}, 1 \mathrm{H}, \mathrm{CHCH}_{2}, J=6.8\right.$ $\mathrm{Hz})$; 3.92-3.95 (m, $\left.1 \mathrm{H}, \mathrm{CHCH}_{2}\right) ; 3.77$ (s, $\left.3 \mathrm{H}, \mathrm{OCH}_{3}\right) ; 2.28$ (s, 3H, $\left.\mathrm{PhCH}_{3}\right)$.

Combined ${ }^{13} \mathrm{C}\left\{{ }^{1} \mathrm{H}\right\}$ NMR: 158.2 (Quat.), 157.7 (Quat.), 152.4 (Quat.), 151.4 (Quat.), 151.2 (Quat.), 143.9 (Quat.), 143.3 (Quat.), 137.7 (Quat.), 136.2 (Quat.), 136.0 (Quat.), 135.7 (Quat.), 135.6 (Quat.), 135.3 (Quat.), 128.7 (Arom. CH), 128.6 (Arom. CH), 128.5 (Arom. CH), 128.32 (Arom. CH), 128.28 (Arom. CH), 128.2 (Arom. CH), 128.14 (Arom. $\mathrm{CH}$ ), 128.06 (Arom. $\mathrm{CH}$ ), 127.5 (Quat.), 125.3 (Arom. CH), 125.1 (Arom. $\mathrm{CH}$ ), 123.0 (Quat.), 119.7 (Quat.), 117.2 (Arom. CH), 117.62 (Arom. CH), 117.55 (Arom. CH), 114.2 (Arom. CH), 114.1 (Arom. CH), 113.9 (Arom. CH), $97.8(\mathrm{COH}), 97.0(\mathrm{COH}), 55.2$ $\left(\mathrm{OCH}_{3}\right), 55.1\left(\mathrm{OCH}_{3}\right), 46.3\left(\mathrm{CHCH}_{2}\right), 42.3\left(\mathrm{CHCH}_{2}\right), 41.6\left(\mathrm{CHCH}_{2}\right), 38.5\left(\mathrm{CHCH}_{2}\right)$, $36.9\left(\mathrm{CHCH}_{2}\right), 20.9(\mathrm{ArCH} 3), 20.8(\mathrm{ArCH} 3), 18.9(\mathrm{ArCH} 3), 16.8(\mathrm{ArCH} 3) . v_{\max }$ $(\mathrm{NaCl}) / \mathrm{cm}^{-1}: 3560,3032,3005,1674,1608,1583,1569,1510,1463,1448,1394,1379$, 1326, 1302, 1246, 1176, 1165, 1083, 1066, 1034. HR-MS: $\mathrm{C}_{24} \mathrm{H}_{23} \mathrm{ClO}_{3}$ Calcd. 394.1336 (M+); found: 394.1340 . 


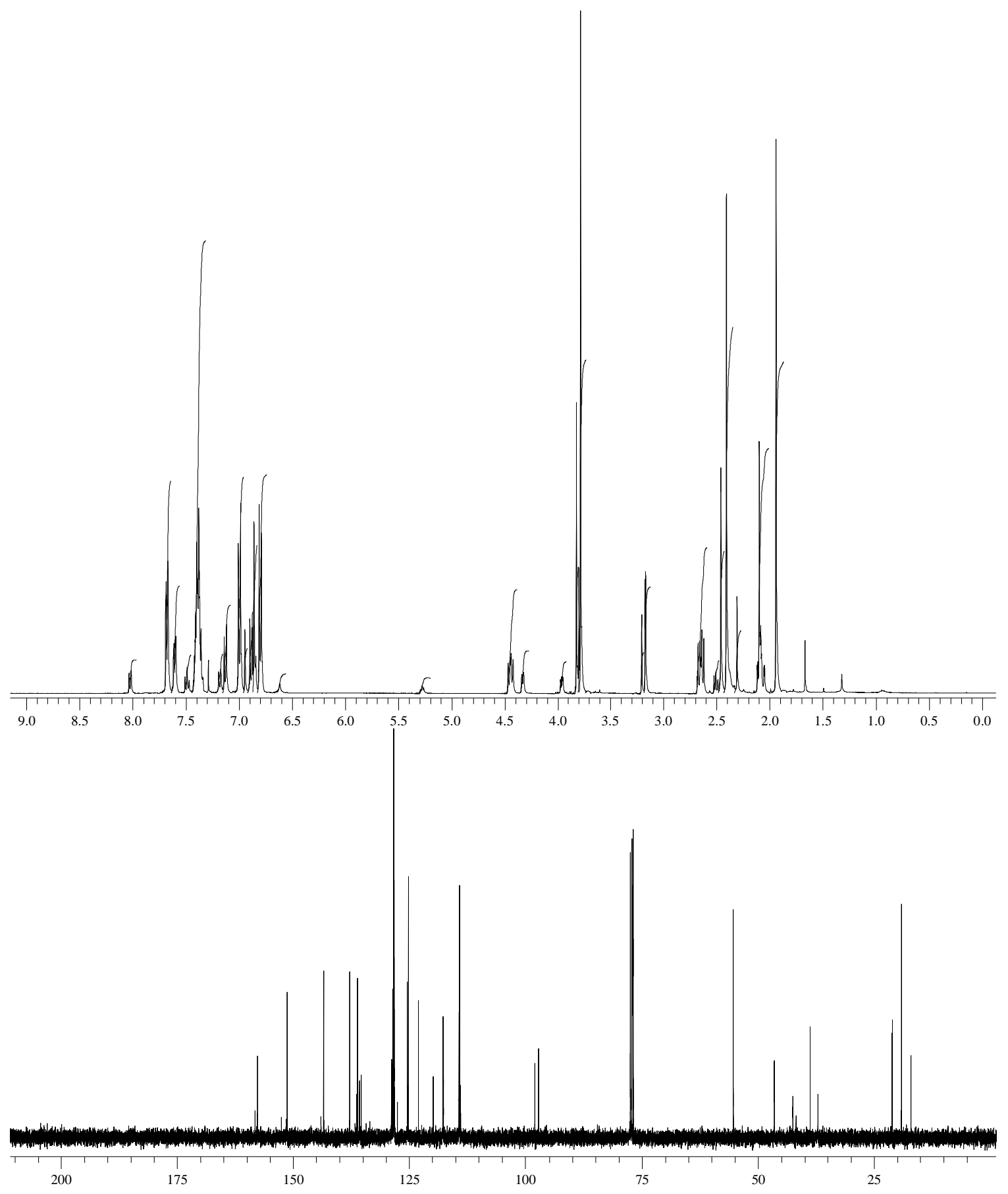




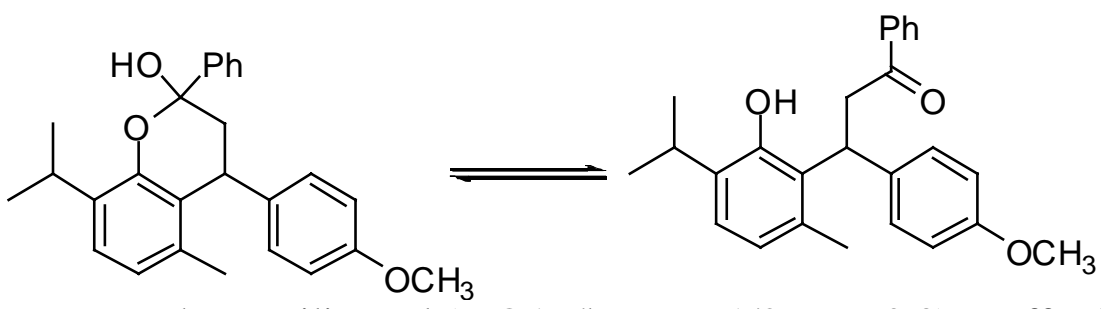

Column chromatography on silica gel (EtOAc/hexane $\left.=1 / 8, R_{\mathrm{f}}=0.3\right)$ to afford $\mathbf{2 e / 3 e}$.

$\mathbf{2 e} / \mathbf{3 e}=18.5: 1, \mathbf{2 e} \mathrm{dr}=1.7: 1$

2e (major) ${ }^{1} \mathrm{H}$ NMR $\left(\mathrm{CDCl}_{3}\right) \delta 7.82(\mathrm{~d}, 2 \mathrm{H}, P h \mathrm{CO}, J=7.3 \mathrm{~Hz}) ; 7.48(\mathrm{t}, 2 \mathrm{H}, P h \mathrm{CO}, J=$ $7.3 \mathrm{~Hz}) ; 7.43-7.46(\mathrm{~m}, 1 \mathrm{H}, P h \mathrm{CO}) ; 7.21\left(\mathrm{~d}, 1 \mathrm{H}, P h \mathrm{CH}_{3}, J=7.8 \mathrm{~Hz}\right) ; 7.11(\mathrm{~d}, 2 \mathrm{H}$, $\left.P h \mathrm{OCH}_{3}, J=8.6 \mathrm{~Hz}\right) ; 6.86\left(\mathrm{~d}, 1 \mathrm{H}, P h \mathrm{CH}_{3}, J=7.8 \mathrm{~Hz}\right) ; 6.85\left(\mathrm{~d}, 2 \mathrm{H}, P h \mathrm{OCH}_{3}, J=8.6\right.$ $\mathrm{Hz}) ; 4.54\left(\mathrm{dd}, 1 \mathrm{H}, \mathrm{CHCH}_{2}, J=11.1\right.$ and $\left.5.3 \mathrm{~Hz}\right) ; 3.81\left(\mathrm{~s}, 3 \mathrm{H}, \mathrm{OCH}_{3}\right) ; 3.54-3.63(\mathrm{~m}, 1 \mathrm{H}$, $\left.\mathrm{CH}\left(\mathrm{CH}_{3}\right)_{2}\right) ; 3.24(\mathrm{~s}, 1 \mathrm{H}, \mathrm{OH}) ; 2.73-2.79\left(\mathrm{~m}, 1 \mathrm{H}, \mathrm{CHCH}_{2}\right) ; 2.20\left(\mathrm{t}, \mathrm{br}, 1 \mathrm{H}, \mathrm{CHCH}_{2}, J=\right.$ $12.6 \mathrm{~Hz}) ; 1.90\left(\mathrm{~s}, 3 \mathrm{H}, \mathrm{PhCH}_{3}\right) ; 1.47\left(\mathrm{~d}, 1 \mathrm{H}, \mathrm{CH}\left(\mathrm{CH}_{3}\right)_{2}, J=6.8 \mathrm{~Hz}\right) ; 1.35(\mathrm{~d}, 1 \mathrm{H}$, $\left.\mathrm{CH}\left(\mathrm{CH}_{3}\right)_{2}, J=6.8 \mathrm{~Hz}\right)$.

2e (minor) ${ }^{1} \mathrm{H}$ NMR $\left(\mathrm{CDCl}_{3}\right) \delta 7.72(\mathrm{~d}, 2 \mathrm{H}, P h \mathrm{CO}, J=7.3 \mathrm{~Hz}) ; 7.46(\mathrm{t}, 2 \mathrm{H}, P h \mathrm{CO}, J=$ $7.3 \mathrm{~Hz}) ; 7.40-7.43(\mathrm{~m}, 1 \mathrm{H}, P h \mathrm{CO}) ; 7.31\left(\mathrm{~d}, 1 \mathrm{H}, P h \mathrm{CH}_{3}, J=7.8 \mathrm{~Hz}\right) ; 7.21(\mathrm{~d}, 2 \mathrm{H}$, $\left.P h \mathrm{OCH}_{3}, J=8.6 \mathrm{~Hz}\right) ; 6.97\left(\mathrm{~d}, 1 \mathrm{H}, P h \mathrm{CH}_{3}, J=7.8 \mathrm{~Hz}\right) ; 6.93\left(\mathrm{~d}, 2 \mathrm{H}, P h \mathrm{OCH}_{3}, J=8.6\right.$ $\mathrm{Hz}) ; 4.39\left(\mathrm{~d}, \mathrm{br}, 1 \mathrm{H}, \mathrm{CHCH}_{2}, J=6.3 \mathrm{~Hz}\right) ; 3.84\left(\mathrm{~s}, 3 \mathrm{H}, \mathrm{OCH}_{3}\right) ; 3.54-3.63(\mathrm{~m}, 1 \mathrm{H}$, $\left.\mathrm{CH}\left(\mathrm{CH}_{3}\right)_{2}\right) ; 3.34(\mathrm{~s}, 1 \mathrm{H}, \mathrm{OH}) ; 2.72-2.78\left(\mathrm{~m}, 1 \mathrm{H}, \mathrm{CHCH}_{2}\right) ; 2.58\left(\mathrm{dd}, 1 \mathrm{H}, \mathrm{CHCH}_{2}, J=\right.$ 14.1 and $7.1 \mathrm{~Hz}) ; 2.08\left(\mathrm{~s}, 3 \mathrm{H}, \mathrm{PhCH}_{3}\right) ; 1.43\left(\mathrm{~d}, 1 \mathrm{H}, \mathrm{CH}\left(\mathrm{CH}_{3}\right)_{2}, J=6.8 \mathrm{~Hz}\right) ; 1.40(\mathrm{~d}, 1 \mathrm{H}$, $\left.\mathrm{CH}\left(\mathrm{CH}_{3}\right)_{2}, J=6.8 \mathrm{~Hz}\right)$.

3e ${ }^{1} \mathrm{H}$ NMR $\left(\mathrm{CDCl}_{3}\right) \delta 8.07(\mathrm{~d}, 2 \mathrm{H}, P h \mathrm{CO}, J=7.3 \mathrm{~Hz}) ; 5.36\left(\mathrm{t}, 1 \mathrm{H}, \mathrm{CHCH}_{2}, J=6.8 \mathrm{~Hz}\right)$; $3.83\left(\mathrm{~s}, 3 \mathrm{H}, \mathrm{OCH}_{3}\right)$.

Combined ${ }^{13} \mathrm{C}\left\{{ }^{1} \mathrm{H}\right\}$ NMR $\delta 157.9$ (Quat.), 157.5 (Quat.), 150.1 (Quat.), 149.9 (Quat.), 144.6 (Quat.), 144.1 (Quat.), 138.2 (Quat.), 135.9 (Quat.), 135.5 (Quat.), 135.2 (Quat.), 134.7 (Quat.), 134.6 (Quat.), 128.8 (Arom. CH), 128.4 (Arom. CH), 128.3 (Arom. CH), 128.2 (Arom. $\mathrm{CH}$ ), 128.1 (Arom. $\mathrm{CH}$ ), 128.0 (Arom. $\mathrm{CH}$ ), 125.3 (Arom. $\mathrm{CH}$ ), 125.1 (Arom. $\mathrm{CH}$ ), 124.4 (Arom. $\mathrm{CH}$ ), 124.0 (Arom. $\mathrm{CH}$ ), 123.6 (Arom. $\mathrm{CH}$ ), 123.5 (Arom. $\mathrm{CH}$ ), 122.5 (Arom. $\mathrm{CH}$ ), 120.2 (Quat.), 114.1 (Arom. $\mathrm{CH}$ ), 113.9 (Arom. $\mathrm{CH}$ ), 97.5 $(\mathrm{COH}), 96.7(\mathrm{COH}), 55.02\left(\mathrm{OCH}_{3}\right), 54.98\left(\mathrm{OCH}_{3}\right), 46.3\left(\mathrm{CHCH}_{2}\right), 42.1\left(\mathrm{CHCH}_{2}\right), 38.3$ $\left(\mathrm{CHCH}_{2}\right), 36.3\left(\mathrm{CHCH}_{2}\right), 26.8\left(\mathrm{CH}\left(\mathrm{CH}_{3}\right)_{2}\right), 26.7\left(\mathrm{CH}\left(\mathrm{CH}_{3}\right)_{2}\right), 26.4\left(\mathrm{CH}\left(\mathrm{CH}_{3}\right)_{2}\right), 23.2$ $\left(\mathrm{CH}\left(\mathrm{CH}_{3}\right)_{2}\right), 22.9\left(\mathrm{CH}\left(\mathrm{CH}_{3}\right)_{2}\right), 22.7\left(\mathrm{CH}\left(\mathrm{CH}_{3}\right)_{2}\right), 22.6\left(\mathrm{CH}\left(\mathrm{CH}_{3}\right)_{2}\right), 21.1\left(\mathrm{ArCH}_{3}\right), 20.5$ $\left(\mathrm{ArCH}_{3}\right), 19.0\left(\mathrm{ArCH}_{3}\right) . v_{\max }(\mathrm{NaCl}) / \mathrm{cm}^{-1}: 3562,3030,3005,1672,1606,1583,1508$, 1463, 1448, 1249, 1176, 1076, 1057, 1034. HR-MS: $\mathrm{C}_{26} \mathrm{H}_{28} \mathrm{O}_{3}$ Calcd. 388.2038 (M+); found: 388.2035 . 

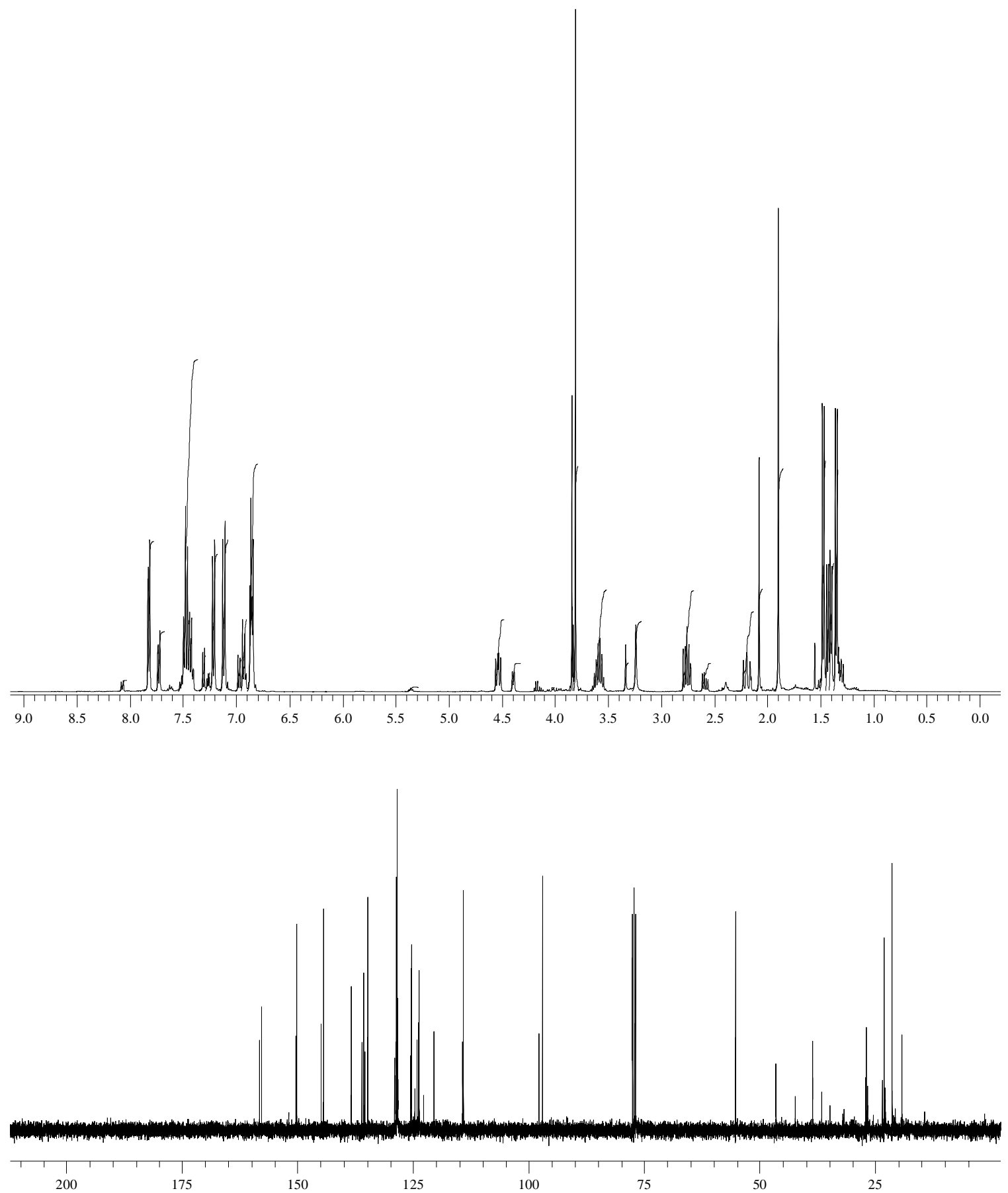

S-11 

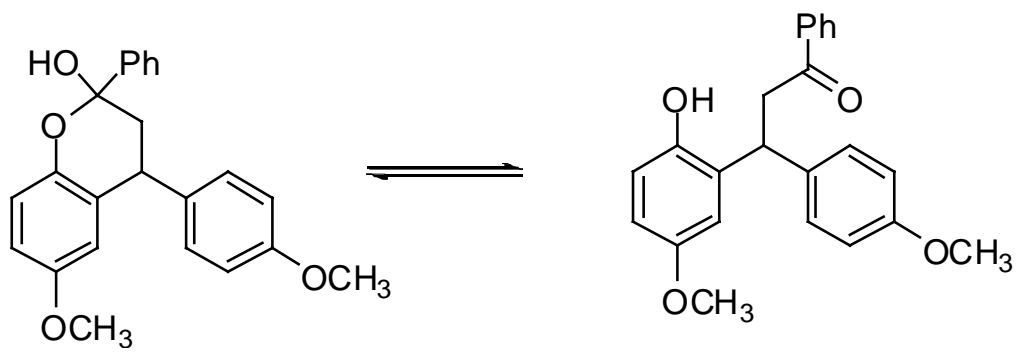

Column chromatography on silica gel (EtOAc/hexane $\left.=1 / 4, R_{\mathrm{f}}=0.2\right)$ to afford $\mathbf{2 f} / \mathbf{3 f}$.

$\mathbf{2 f} / \mathbf{3 f}=0.48: 1, \mathbf{2 f} \mathrm{dr}=8: 1$

2f (major) ${ }^{1} \mathrm{H}$ NMR $\left(\mathrm{CDCl}_{3}\right) \delta 7.72(\mathrm{~d}, 2 \mathrm{H}, P h \mathrm{CO}, J=7.0 \mathrm{~Hz}) ; 7.57$ (t, $2 \mathrm{H}, P h \mathrm{CO}, J=$ $7.0 \mathrm{~Hz}) ; 7.36(\mathrm{t}, 1 \mathrm{H}, P h \mathrm{CO}, J=7.0 \mathrm{~Hz}) ; 7.17\left(\mathrm{~d}, 2 \mathrm{H}, P h \mathrm{OCH}_{3}, J=8.6 \mathrm{~Hz}\right) ; 7.01(\mathrm{~d}, 1 \mathrm{H}$, $\left.P h \mathrm{OCH}_{3}, J=8.8 \mathrm{~Hz}\right) ; 6.87\left(\mathrm{~d}, 1 \mathrm{H}, P h \mathrm{OCH}_{3}, J=8.8 \mathrm{~Hz}\right) ; 6.83\left(\mathrm{~d}, 2 \mathrm{H}, P h \mathrm{OCH}_{3}, J=8.6\right.$ $\mathrm{Hz}) ; 6.39\left(\mathrm{~d}, 1 \mathrm{H}, \mathrm{PhOCH}_{3}, J=3.0 \mathrm{~Hz}\right) ; 4.45\left(\mathrm{dd}, 1 \mathrm{H}, \mathrm{CHCH}_{2}, J=13.1\right.$ and $\left.5.8 \mathrm{~Hz}\right) ; 3.78$ (s, $\left.3 \mathrm{H}, \mathrm{OCH}_{3}\right) ; 3.76\left(\mathrm{~s}, 3 \mathrm{H}, \mathrm{OCH}_{3}\right) ; 3.41(\mathrm{~s}, \mathrm{br}, 1 \mathrm{H}, \mathrm{OH}) ; 2.45\left(\mathrm{dd}, 1 \mathrm{H}, \mathrm{CHCH}_{2}, J=13.6\right.$ and $5.6 \mathrm{~Hz}) ; 2.08\left(\mathrm{t}, 1 \mathrm{H}, \mathrm{CHCH}_{2}, J=12.9 \mathrm{~Hz}\right)$.

$2 f$ (minor) ${ }^{1} \mathrm{H} \mathrm{NMR}\left(\mathrm{CDCl}_{3}\right) \delta 7.08\left(\mathrm{~d}, 2 \mathrm{H}, P h \mathrm{OCH}_{3}, J=8.6 \mathrm{~Hz}\right) ; 6.30\left(\mathrm{~d}, 1 \mathrm{H}, P h \mathrm{OCH}_{3}\right.$, $J=3.0 \mathrm{~Hz}) ; 2.66\left(\mathrm{dd}, 1 \mathrm{H}, \mathrm{CHCH}_{2}, J=13.6\right.$ and $\left.5.8 \mathrm{~Hz}\right) ; 2.50\left(\mathrm{dd}, 1 \mathrm{H}, \mathrm{CHCH}_{2}, J=13.4\right.$ and $9.9 \mathrm{~Hz}) ; 3.80\left(\mathrm{~s}, 3 \mathrm{H}, \mathrm{OCH}_{3}\right) ; 3.64\left(\mathrm{~s}, 3 \mathrm{H}, \mathrm{OCH}_{3}\right)$.

3f ${ }^{1} \mathrm{H}$ NMR $\left(\mathrm{CDCl}_{3}\right): 7.99$ (d, 2H, $\left.P h \mathrm{CO}, J=7.0 \mathrm{~Hz}\right) ; 7.45(\mathrm{t}, 2 \mathrm{H}, P h \mathrm{CO}, J=7.0 \mathrm{~Hz})$; $7.41(\mathrm{t}, 1 \mathrm{H}, P h \mathrm{CO}, J=7.0 \mathrm{~Hz}) ; 7.26\left(\mathrm{~d}, 2 \mathrm{H}, P h \mathrm{OCH}_{3}, J=8.6 \mathrm{~Hz}\right) ; 6.93\left(\mathrm{~d}, 1 \mathrm{H}, P h \mathrm{OCH}_{3}\right.$, $J=8.8 \mathrm{~Hz}) ; 6.85\left(\mathrm{~d}, 2 \mathrm{H}, P h \mathrm{OCH}_{3}, J=8.6 \mathrm{~Hz}\right) ; 6.61\left(\mathrm{~d}, 1 \mathrm{H}, P h \mathrm{OCH}_{3}, J=8.8 \mathrm{~Hz}\right) ; 6.60$ $\left(\mathrm{s}, 1 \mathrm{H}, \mathrm{PhOCH}_{3}\right) ; 5.02\left(\mathrm{dd}, 1 \mathrm{H}, \mathrm{CHCH}_{2}, J=9.3\right.$ and $\left.5.1 \mathrm{~Hz}\right) ; 3.88\left(\mathrm{dd}, 1 \mathrm{H}, \mathrm{CHCH}_{2}, J=\right.$ 17.7 and $9.1 \mathrm{~Hz}) ; 3.76\left(\mathrm{~s}, 3 \mathrm{H}, \mathrm{OCH}_{3}\right) ; 3.71\left(\mathrm{dd}, 1 \mathrm{H}, \mathrm{CHCH}_{2}, J=11.6\right.$ and $\left.6.8 \mathrm{~Hz}\right) ; 3.65$ (s, $3 \mathrm{H}, \mathrm{OCH}_{3}$ ).

Combined ${ }^{13} \mathrm{C}\left\{{ }^{1} \mathrm{H}\right\}$ NMR $\delta 200.2(\mathrm{C}=\mathrm{O}), 158.3$ (Quat.), 158.0 (Quat.), 153.72 (Quat.), 153.66 (Quat.), 153.4 (Quat.), 149.6 (Quat.), 147.2 (Quat.), 146.6 (Quat.), 144.1 (Quat.), 136.3 (Quat.), 135.5 (Quat.), 135.3 (Quat.), 133.6 (Arom. $\mathrm{CH}$ ), 133.0 (Quat.), 129.8 (Quat.), 128.8 (Arom. CH), 128.6 (Arom. CH), 128.4 (Arom. CH), 128.31 (Arom. CH), 128.25 (Arom. CH), 126.8 (Quat.), 125.2 (Arom. CH), 118.2 (Quat.), 117.7 (Quat.), 116.0 (Arom. CH), 114.8 (Arom. CH), 114.6 (Arom. CH), 114.0 (Arom. $\mathrm{CH}$ ), 113.6 (Arom. CH), 111.9 (Quat.), $97.4(\mathrm{COH}), 55.7\left(\mathrm{OCH}_{3}\right), 55.6\left(\mathrm{OCH}_{3}\right), 55.5\left(\mathrm{OCH}_{3}\right), 55.21$ $\left(\mathrm{OCH}_{3}\right), 55.17\left(\mathrm{OCH}_{3}\right), 44.7\left(\mathrm{CHCH}_{2}\right), 43.2\left(\mathrm{CHCH}_{2}\right), 38.5\left(\mathrm{CHCH}_{2}\right), 37.5\left(\mathrm{CHCH}_{2}\right)$. $v_{\max }(\mathrm{NaCl}) / \mathrm{cm}^{-1}: 3571,3365,3033,3003,1674,1610,1596,1512,1492,1448,1272$, 1249, 1205, 1180, 1036. HR-MS: $\mathrm{C}_{23} \mathrm{H}_{26} \mathrm{O}_{4} \mathrm{~N}$ Calcd. $380.1862\left(\mathrm{M}+\mathrm{NH}_{4}\right)$; found: 380.1852 . 


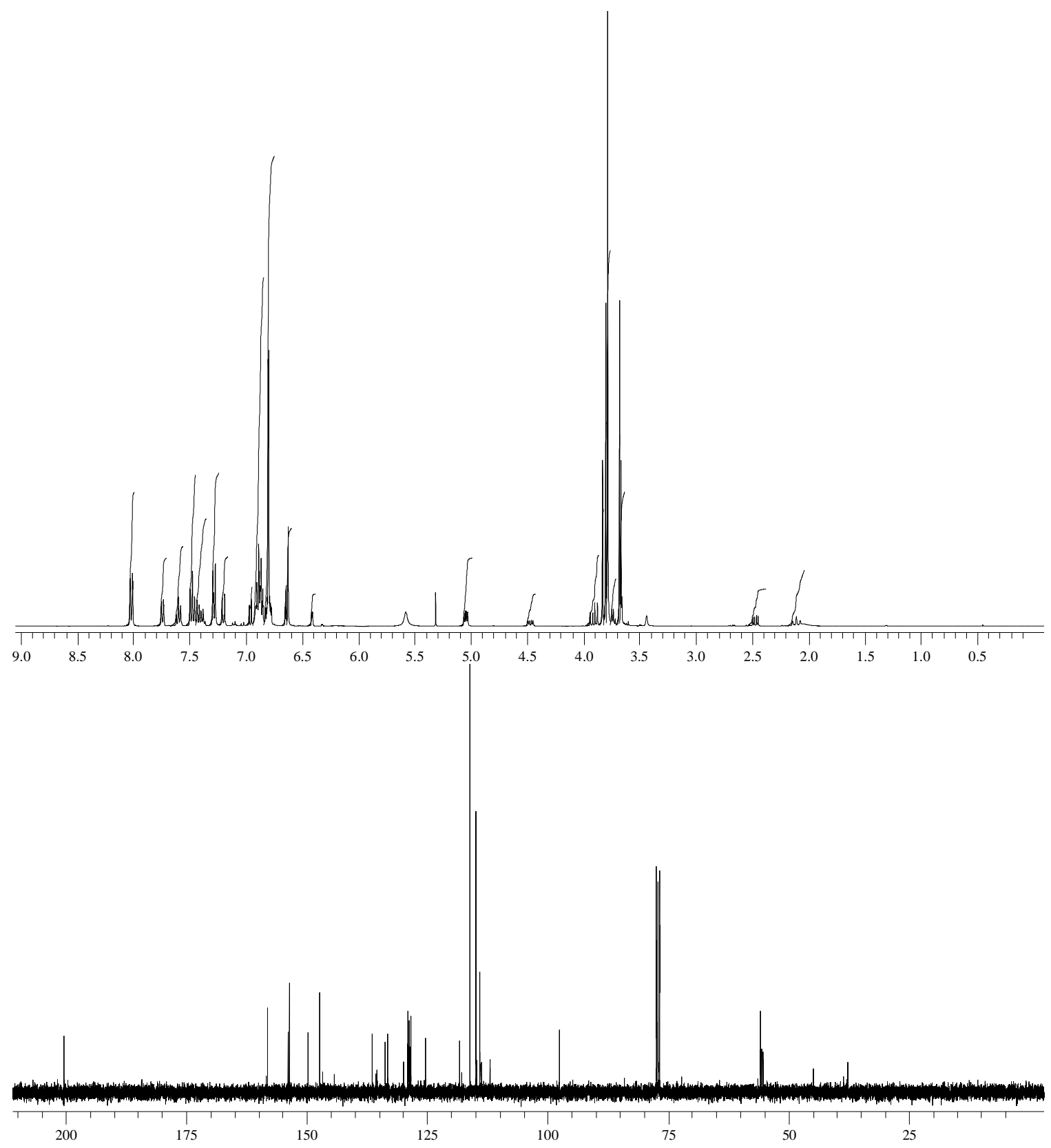

S-13 


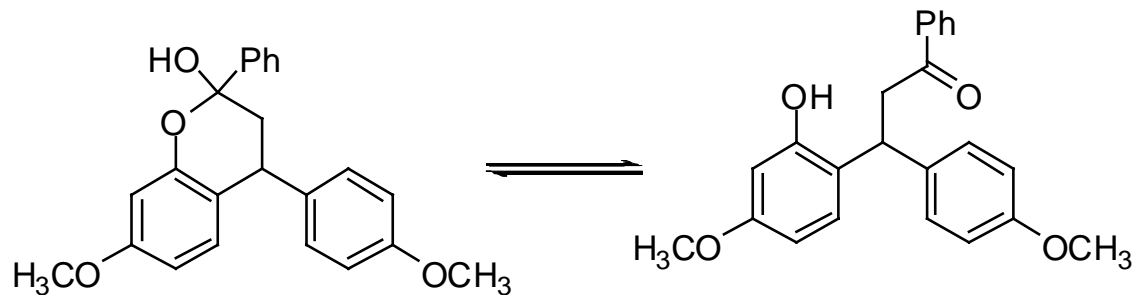

Column chromatography on silica gel $\left(\right.$ EtOAc/hexane $\left.=1 / 4, R_{\mathrm{f}}=0.2\right)$ to afford $\mathbf{2 g} / \mathbf{3 g}$.

$\mathbf{2 g} / \mathbf{3 g}=0.47: 1, \mathbf{2 g} \mathrm{dr}=7.8: 1$

$2 \mathrm{~g}$ (major) ${ }^{1} \mathrm{H} \mathrm{NMR}\left(\mathrm{CDCl}_{3}\right) \delta 7.72(\mathrm{~d}, 2 \mathrm{H}, P h \mathrm{CO}, J=7.3 \mathrm{~Hz}) ; 7.58(\mathrm{t}, 2 \mathrm{H}, P h \mathrm{CO}, J=$ $7.3 \mathrm{~Hz}) ; 7.37(\mathrm{t}, 1 \mathrm{H}, P h \mathrm{CO}, J=7.3 \mathrm{~Hz}) ; 7.18\left(\mathrm{~d}, 2 \mathrm{H}, P h \mathrm{OCH}_{3}, J=8.6 \mathrm{~Hz}\right) ; 6.87(\mathrm{~d}, 2 \mathrm{H}$, $\left.P h \mathrm{OCH}_{3}, J=8.6 \mathrm{~Hz}\right) ; 6.72\left(\mathrm{~d}, 1 \mathrm{H}, P h \mathrm{OCH}_{3}, J=8.6 \mathrm{~Hz}\right) ; 6.58\left(\mathrm{~d}, 1 \mathrm{H}, P h \mathrm{OCH}_{3}, J=2.8\right.$ $\mathrm{Hz}) ; 6.47\left(\mathrm{dd}, 1 \mathrm{H}, P \mathrm{OCH}_{3}, J=8.6\right.$ and $\left.2.8 \mathrm{~Hz}\right) ; 4.40\left(\mathrm{dd}, 1 \mathrm{H}, \mathrm{CHCH}_{2}, J=12.9\right.$ and 5.6 $\mathrm{Hz}) ; 3.80\left(\mathrm{~s}, 3 \mathrm{H}, \mathrm{OCH}_{3}\right) ; 3.77$ (s, $\left.3 \mathrm{H}, \mathrm{OCH}_{3}\right) ; 3.40(\mathrm{~d}, 1 \mathrm{H}, \mathrm{OH}, J=2.5 \mathrm{~Hz}) ; 2.44(\mathrm{dd}, 1 \mathrm{H}$, $\mathrm{CHCH}_{2}, J=13.6$ and $\left.5.6 \mathrm{~Hz}\right) ; 2.07$ (dd, $1 \mathrm{H}, \mathrm{CHCH}_{2}, J=13.4$ and $2.3 \mathrm{~Hz}$ ).

$2 \mathrm{~g}$ (minor) ${ }^{1} \mathrm{H}$ NMR $\left(\mathrm{CDCl}_{3}\right) \delta 7.08\left(\mathrm{~d}, 2 \mathrm{H}, \mathrm{PhOCH}_{3}, J=8.6 \mathrm{~Hz}\right) ; 2.64\left(\mathrm{dd}, 1 \mathrm{H}, \mathrm{CHCH}_{2}\right.$, $J=13.4$ and $5.6 \mathrm{~Hz}) ; 2.49\left(\mathrm{dd}, 1 \mathrm{H}, \mathrm{CHCH}_{2}, J=13.4\right.$ and $\left.9.9 \mathrm{~Hz}\right)$.

$3 \mathrm{~g}{ }^{1} \mathrm{H}$ NMR $\left(\mathrm{CDCl}_{3}\right) \delta 8.00(\mathrm{~d}, 2 \mathrm{H}, P h \mathrm{CO}, J=7.3 \mathrm{~Hz}) ; 7.46(\mathrm{t}, 2 \mathrm{H}, P h \mathrm{CO}, J=7.3 \mathrm{~Hz})$; 7.40 (t, $1 \mathrm{H}, P h \mathrm{CO}, J=7.3 \mathrm{~Hz}) ; 7.25\left(\mathrm{~d}, 2 \mathrm{H}, P h \mathrm{OCH}_{3}, J=8.6 \mathrm{~Hz}\right) ; 6.89$ (d, $1 \mathrm{H}, P h \mathrm{OCH}_{3}$, $J=8.6 \mathrm{~Hz}) ; 6.86\left(\mathrm{~d}, 2 \mathrm{H}, P h \mathrm{OCH}_{3}, J=8.6 \mathrm{~Hz}\right) ; 6.46\left(\mathrm{~d}, 1 \mathrm{H}, P h \mathrm{OCH}_{3}, J=2.5 \mathrm{~Hz}\right) ; 6.39$ $\left(\mathrm{dd}, 1 \mathrm{H}, P \mathrm{OCH}_{3}, J=8.6\right.$ and $\left.2.5 \mathrm{~Hz}\right) ; 4.95\left(\mathrm{dd}, 1 \mathrm{H}, \mathrm{CHCH}_{2}, J=9.3\right.$ and $\left.4.5 \mathrm{~Hz}\right) ; 3.88$ $\left(\mathrm{dd}, 1 \mathrm{H}, \mathrm{CHCH}_{2}, J=17.7\right.$ and $\left.9.3 \mathrm{~Hz}\right) ; 3.78\left(\mathrm{~s}, 3 \mathrm{H}, \mathrm{OCH}_{3}\right) ; 3.78\left(\mathrm{dd}, 1 \mathrm{H}, \mathrm{CHCH}_{2}, J=\right.$ 17.9 and $4.5 \mathrm{~Hz}) ; 3.70\left(\mathrm{~s}, 3 \mathrm{H}, \mathrm{OCH}_{3}\right)$.

Combined ${ }^{13} \mathrm{C}\left\{{ }^{1} \mathrm{H}\right\}$ NMR $\delta 200.4(\mathrm{C}=\mathrm{O}), 159.2$ (Quat.), 159.0 (Quat.), 158.3 (Quat.), 158.0 (Quat.), 154.4 (Quat.), 153.2 (Quat.), 144.0 (Quat.), 136.4 (Quat.), 135.9 (Quat.), 135.8 (Quat.), 133.6 (Arom. CH), 129.9 (Arom. CH), 129.7 (Arom. CH), 129.1 (Arom. $\mathrm{CH}$ ), 128.8 (Arom. $\mathrm{CH}$ ), 128.6 (Arom. $\mathrm{CH}$ ), 128.5 (Arom. $\mathrm{CH}$ ), 128.3 (Arom. $\mathrm{CH}$ ), 128.2 (Arom. $\mathrm{CH}$ ), 125.2 (Arom. $\mathrm{CH}$ ), 123.9 (Quat.), 118.4 (Quat.), 113.92 (Arom. $\mathrm{CH}$ ), 113.89 (Arom. $\mathrm{CH}$ ), 107.8 (Arom. $\mathrm{CH}$ ), 107.0 (Arom. $\mathrm{CH}$ ), 102.8 (Arom. $\mathrm{CH}$ ), 101.9 (Arom. CH), $97.8(\mathrm{COH}), 55.24\left(\mathrm{OCH}_{3}\right), 55.16\left(\mathrm{OCH}_{3}\right), 55.1\left(\mathrm{OCH}_{3}\right), 44.9\left(\mathrm{CHCH}_{2}\right)$, $43.4\left(\mathrm{CHCH}_{2}\right), 37.6\left(\mathrm{CHCH}_{2}\right), 36.7\left(\mathrm{CHCH}_{2}\right) . v_{\max }(\mathrm{NaCl}) / \mathrm{cm}^{-1}: 3569,3323,3033,3006$, 1674, 1618, 1581, 1512, 1448, 1303, 1247, 1203, 1180, 1159, 1036. HR-MS: $\mathrm{C}_{23} \mathrm{H}_{26} \mathrm{O}_{4} \mathrm{~N}$ Calcd. $380.1862\left(\mathrm{M}+\mathrm{NH}_{4}\right)$; found: 380.1859 . 


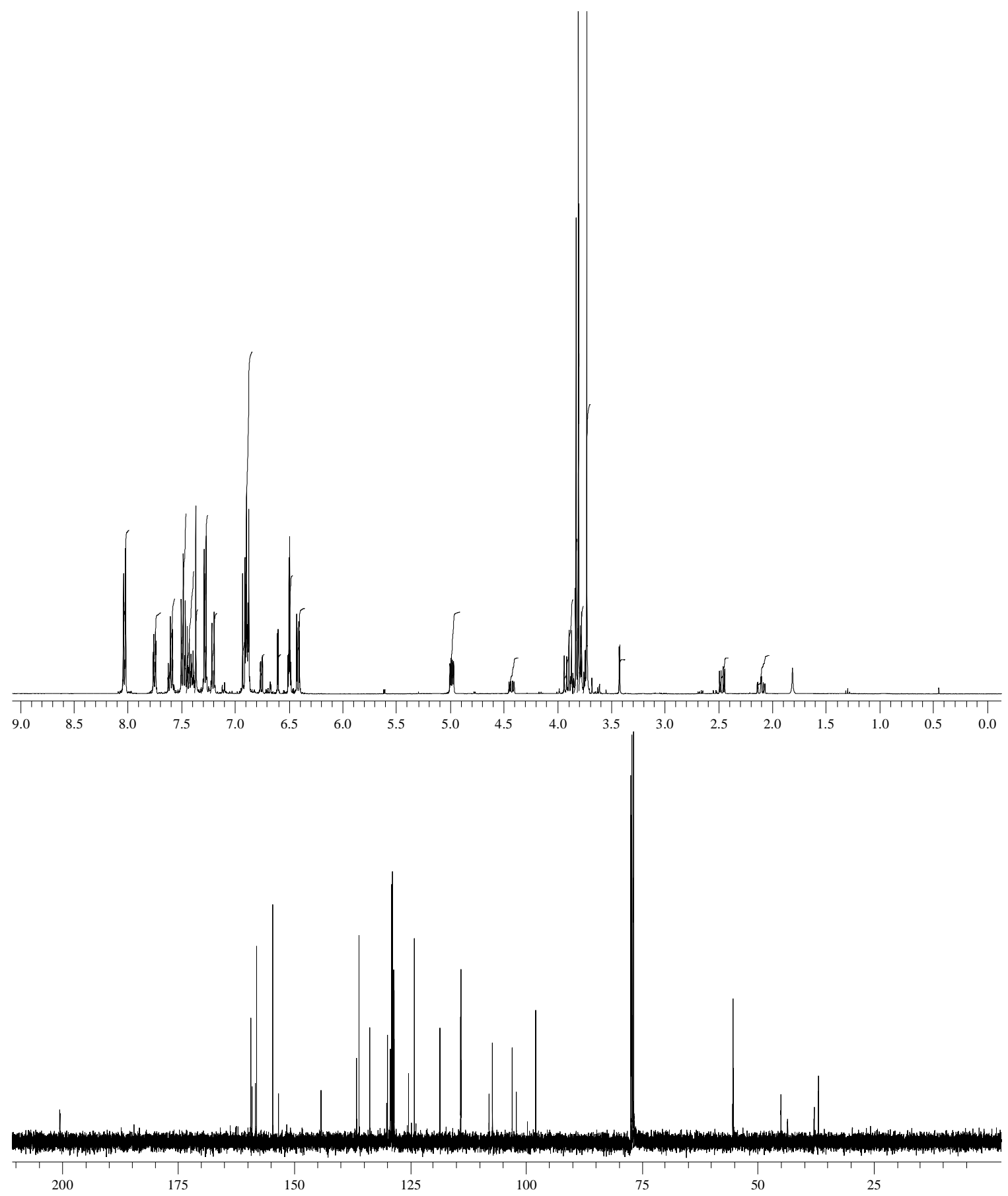

S-15 


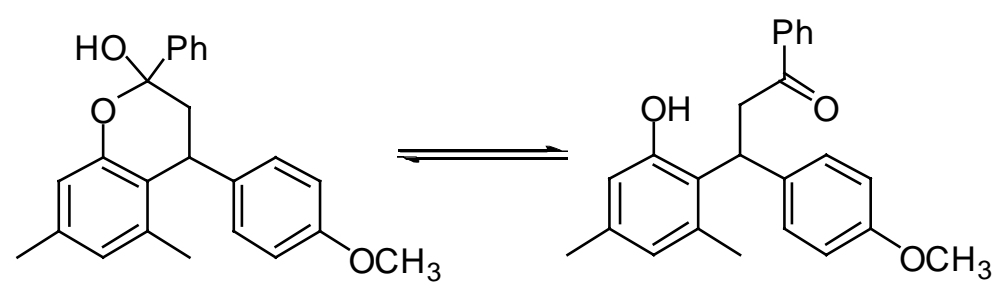

Column chromatography on silica gel $\left(\right.$ EtOAc/hexane $=1 / 4, \mathrm{R}_{\mathrm{f}}=0.3$ ) to afford $\mathbf{2 h} / \mathbf{3 h}$.

$\mathbf{2 h} / \mathbf{3 h}=2.4: 1, \mathbf{2 h} \mathrm{dr}=2: 1$

2h (major) ${ }^{1} \mathrm{H}$ NMR $\left(\mathrm{CDCl}_{3}\right) \delta 7.69$ (d, 2H, PhCO, J = $\left.7.3 \mathrm{~Hz}\right) ; 7.39$ (t, 2H, PhCO, $J=$ $7.3 \mathrm{~Hz}) ; 7.36-7.38(\mathrm{~m}, 1 \mathrm{H}, P h \mathrm{CO}) ; 7.04\left(\mathrm{~d}, 2 \mathrm{H}, P h \mathrm{OCH}_{3}, J=8.6 \mathrm{~Hz}\right) ; 6.79(\mathrm{~d}, 2 \mathrm{H}$, $\left.P h \mathrm{OCH}_{3}, J=8.6 \mathrm{~Hz}\right) ; 6.76\left(\mathrm{~s}, 1 \mathrm{H}, P h \mathrm{CH}_{3}\right) ; 6.64\left(\mathrm{~s}, 1 \mathrm{H}, P h \mathrm{CH}_{3}\right) ; 4.38\left(\mathrm{dd}, 1 \mathrm{H}, \mathrm{CHCH}_{2}, J\right.$ $=11.1$ and $7.3 \mathrm{~Hz}) ; 3.77\left(\mathrm{~s}, 3 \mathrm{H}, \mathrm{OCH}_{3}\right) ; 3.12(\mathrm{~d}, 1 \mathrm{H}, \mathrm{OH}, J=2.0 \mathrm{~Hz}) ; 2.60-2.65(\mathrm{~m}, 1 \mathrm{H}$, $\left.\mathrm{CHCH}_{2}\right) ; 2.32$ (s, 3H, $\left.\mathrm{PhCH}{ }_{3}\right) ; 2.07-2.14\left(\mathrm{~m}, 1 \mathrm{H}, \mathrm{CHCH}_{2}\right) ; 1.80\left(\mathrm{~s}, 3 \mathrm{H}, \mathrm{PhCH}_{3}\right)$.

2h (minor) ${ }^{1} \mathrm{H}$ NMR $\left(\mathrm{CDCl}_{3}\right) \delta 7.61(\mathrm{~d}, 2 \mathrm{H}, P h \mathrm{CO}, J=7.3 \mathrm{~Hz}) ; 7.57$ (t, 2H,PhCO, $J=$ $7.3 \mathrm{~Hz}) ; 7.35-7.37(\mathrm{~m}, 1 \mathrm{H}, P h \mathrm{CO}) ; 7.14\left(\mathrm{~d}, 2 \mathrm{H}, P h \mathrm{OCH}_{3}, J=8.6 \mathrm{~Hz}\right) ; 6.83(\mathrm{~d}, 2 \mathrm{H}$, $\left.P h \mathrm{OCH}_{3}, J=8.8 \mathrm{~Hz}\right) ; 6.85\left(\mathrm{~s}, 1 \mathrm{H}, P h \mathrm{CH}_{3}\right) ; 6.60\left(\mathrm{~s}, 1 \mathrm{H}, P h \mathrm{CH}_{3}\right) ; 4.27(\mathrm{~d}, \mathrm{br}, 1 \mathrm{H}$, $\left.\mathrm{CHCH}_{2}, J=5.6 \mathrm{~Hz}\right) ; 3.81\left(\mathrm{~s}, 3 \mathrm{H}, \mathrm{OCH}_{3}\right) ; 3.21(\mathrm{~s}, 1 \mathrm{H}, \mathrm{OH}) ; 2.62-2.66\left(\mathrm{~m}, 1 \mathrm{H}, \mathrm{CHCH}_{2}\right)$; $2.50\left(\mathrm{dd}, 1 \mathrm{H}, \mathrm{CHCH}_{2}, J=14.1\right.$ and $\left.6.8 \mathrm{~Hz}\right) ; 2.38\left(\mathrm{~s}, 3 \mathrm{H}, \mathrm{PhCH}_{3}\right) ; 2.07-2.14(\mathrm{~m}, 1 \mathrm{H}$, $\left.\mathrm{CHCH}_{2}\right) ; 1.99\left(\mathrm{~s}, 3 \mathrm{H}, \mathrm{PhCH}_{3}\right)$.

3h ${ }^{1} \mathrm{H}$ NMR $\left(\mathrm{CDCl}_{3}\right) \delta 8.01(\mathrm{~d}, 2 \mathrm{H}, P h \mathrm{CO}, J=7.3 \mathrm{~Hz}) ; 7.46(\mathrm{t}, 2 \mathrm{H}, P h \mathrm{CO}, J=7.3 \mathrm{~Hz})$; 7.33-7.35 (m, $1 \mathrm{H}, P h \mathrm{CO}) ; 7.22\left(\mathrm{~d}, 2 \mathrm{H}, P h \mathrm{OCH}_{3}, J=8.8 \mathrm{~Hz}\right) ; 6.87\left(\mathrm{~d}, 2 \mathrm{H}, P h \mathrm{OCH}_{3}, J=\right.$ $8.6 \mathrm{~Hz}) ; 6.75\left(\mathrm{~s}, 1 \mathrm{H}, P h \mathrm{CH}_{3}\right) ; 6.46\left(\mathrm{~s}, 1 \mathrm{H}, P h \mathrm{CH}_{3}\right) ; 5.17\left(\mathrm{t}, 1 \mathrm{H}, \mathrm{CHCH}_{2}, J=7.1 \mathrm{~Hz}\right)$; $3.94\left(\mathrm{t}, 2 \mathrm{H}, \mathrm{CHCH}_{2}, J=7.1 \mathrm{~Hz}\right) ; 3.77\left(\mathrm{~s}, 3 \mathrm{H}, \mathrm{OCH}_{3}\right) ; 2.34\left(\mathrm{~s}, 3 \mathrm{H}, \mathrm{PhCH}_{3}\right) ; 2.22$ (s, 3H, $\left.\mathrm{PhCH}_{3}\right)$.

Combined ${ }^{13} \mathrm{C}\left\{{ }^{1} \mathrm{H}\right\}$ NMR $\delta 199.8$ (C=O), 158.0 (Quat.), 157.8 (Quat.), 157.6 (Quat.), 154.2 (Quat.), 153.2 (Quat.), 153.0 (Quat.), 144.2 (Quat.), 143.7 (Quat.), 138.3 (Quat.), 138.0 (Quat.), 137.95 (Quat.), 137.9 (Quat.), 137.6 (Quat.), 137.3 (Quat.), 137.2 (Quat.), 136.8 (Quat.), 135.6 (Quat.), 134.8 (Quat.), 133.1 (Arom. CH), 128.7 (Arom. CH), 128.5 (Arom. CH), 128.34 (Arom. CH), 128.32 (Arom. CH), 128.3 (Arom. CH), 128.2 (Arom. $\mathrm{CH}$ ), 128.14 (Arom. CH), 128.13 (Arom. CH), 128.11 (Arom. $\mathrm{CH}$ ), 126.2 (Quat.), 125.4 (Arom. CH), 125.2 (Arom. CH), 125.18 (Arom. CH), 124.4 (Arom. CH), 124.1 (Arom. $\mathrm{CH}$ ), 120.8 (Quat.), 116.1 (Arom. CH), 115.91 (Arom. CH), 115.89 (Arom. CH), 114.1 (Arom. CH), 113.9 (Arom. CH), 113.8 (Arom. $\mathrm{CH}$ ), $97.8(\mathrm{COH}), 97.0(\mathrm{COH}), 55.13$ $\left(\mathrm{OCH}_{3}\right), 55.11\left(\mathrm{OCH}_{3}\right), 55.08\left(\mathrm{OCH}_{3}\right), 46.3\left(\mathrm{CHCH}_{2}\right), 42.4\left(\mathrm{CHCH}_{2}\right), 41.7\left(\mathrm{CHCH}_{2}\right)$, $37.8\left(\mathrm{CHCH}_{2}\right), 35.8\left(\mathrm{CHCH}_{2}\right), 21.2\left(\mathrm{ArCH}_{3}\right), 21.0\left(\mathrm{ArCH}_{3}\right), 20.9\left(\mathrm{ArCH}_{3}\right), 20.7$ $\left.\left(\mathrm{ArCH}_{3}\right), 20.6\left(\mathrm{ArCH}_{3}\right), 19.1(\mathrm{ArCH})_{3}\right) \cdot v_{\max }(\mathrm{NaCl}) / \mathrm{cm}^{-1}: 3564,3030,3005,1683,1610$, 1579, 1510, 1462, 1448, 1301, 1248, 1178, 1066, 1034. HR-MS: $\mathrm{C}_{24} \mathrm{H}_{24} \mathrm{O}_{3} \mathrm{Na}$ Calcd. $383.1623(\mathrm{M}+\mathrm{Na})$; found: 383.1607 . 


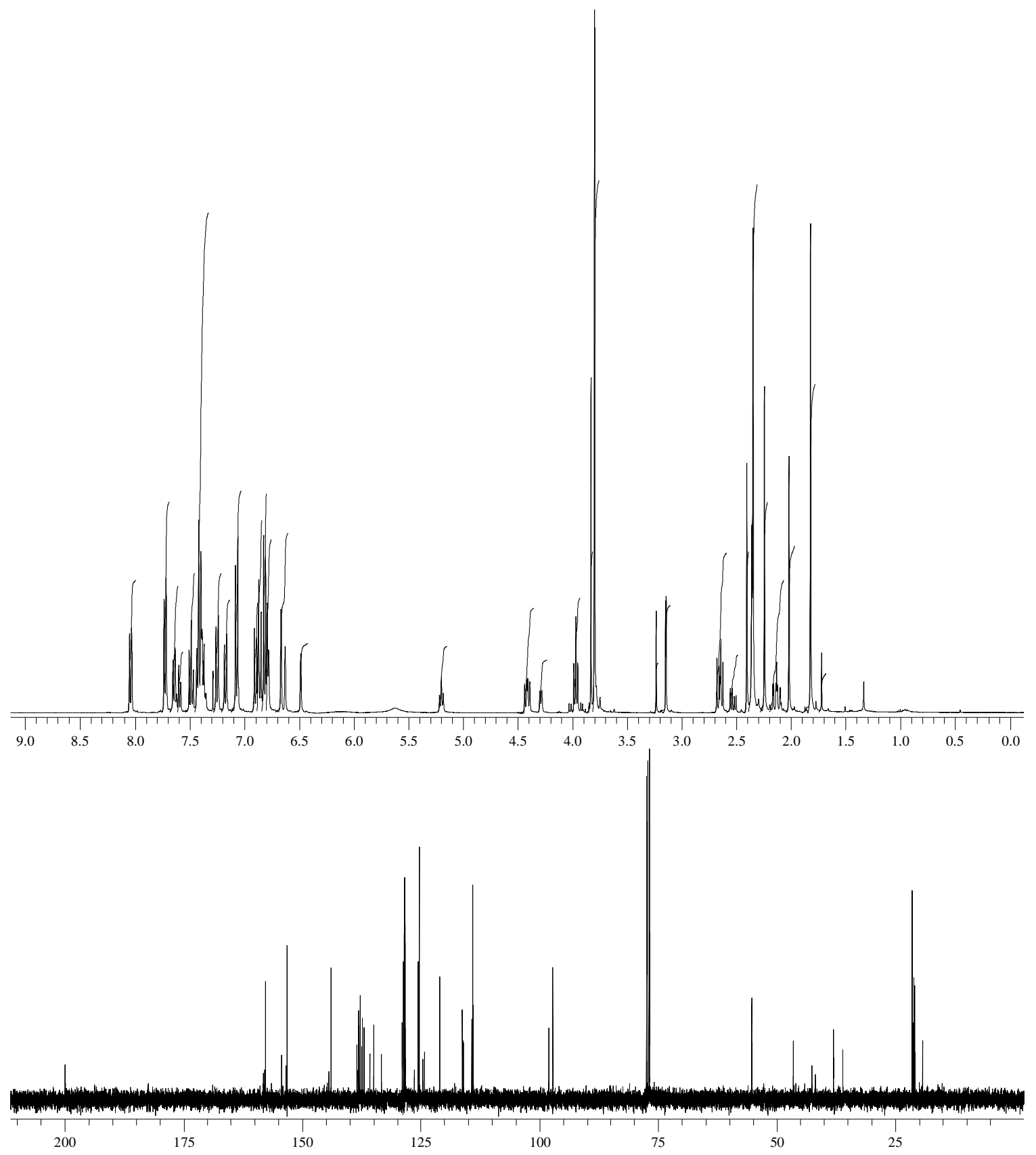

S-17 


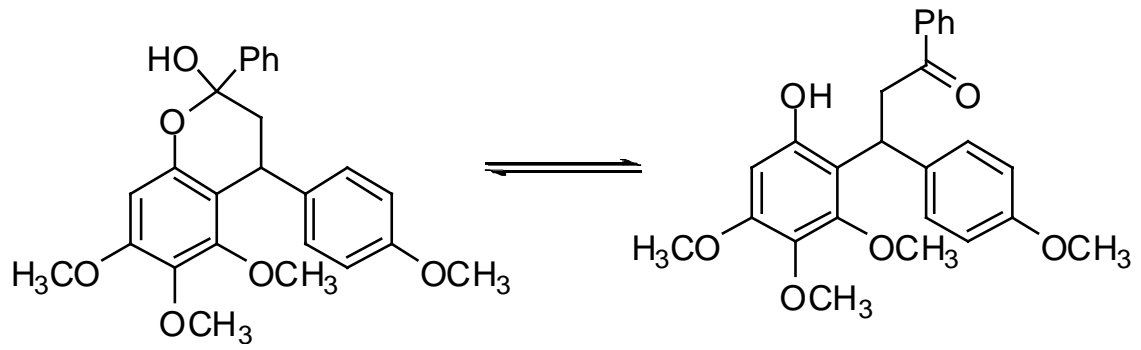

Column chromatography on silica gel (EtOAc/hexane $\left.=1 / 4, \mathbf{R}_{\mathrm{f}}=0.2\right)$ to afford $\mathbf{2} \mathbf{i} / \mathbf{3 i}$.

$2 \mathbf{i} / \mathbf{3 i}=0.52: 1,2 \mathbf{i} \mathrm{dr}=1.9: 1$

$2 \mathbf{i}$ (major) ${ }^{1} \mathrm{H}$ NMR $\left(\mathrm{CDCl}_{3}\right) \delta 7.68(\mathrm{~d}, 2 \mathrm{H}, P h \mathrm{CO}, J=7.3 \mathrm{~Hz}) ; 7.57(\mathrm{t}, 2 \mathrm{H}, P h \mathrm{CO}, J=$ $7.3 \mathrm{~Hz}) ; 7.35$ (t, $1 \mathrm{H}, P h \mathrm{CO}, J=7.3 \mathrm{~Hz}) ; 7.12\left(\mathrm{~d}, 2 \mathrm{H}, P h \mathrm{OCH}_{3}, J=8.6 \mathrm{~Hz}\right) ; 6.80(\mathrm{~d}, 2 \mathrm{H}$,

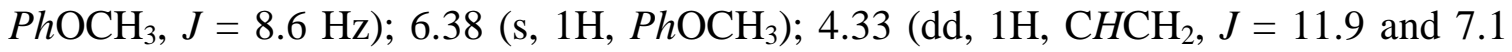
$\mathrm{Hz}) ; 3.84\left(\mathrm{~s}, 3 \mathrm{H}, \mathrm{OCH}_{3}\right) ; 3.76\left(\mathrm{~s}, 3 \mathrm{H}, \mathrm{OCH}_{3}\right) ; 3.73\left(\mathrm{~s}, 3 \mathrm{H}, \mathrm{OCH}_{3}\right) ; 3.17(\mathrm{~d}, 1 \mathrm{H}, \mathrm{OH}, J=$ $2.3 \mathrm{~Hz}) ; 3.16\left(\mathrm{~s}, 3 \mathrm{H}, \mathrm{OCH}_{3}\right) ; 2.51\left(\mathrm{dd}, 1 \mathrm{H}, \mathrm{CHCH}_{2}, J=13.9\right.$ and $\left.7.1 \mathrm{~Hz}\right) ; 1.97-2.10(\mathrm{~m}$, $\left.1 \mathrm{H}, \mathrm{CHCH}_{2}\right)$.

$2 \mathbf{i}$ (minor) ${ }^{1} \mathrm{H} \mathrm{NMR}\left(\mathrm{CDCl}_{3}\right) \delta 7.56(\mathrm{~d}, 2 \mathrm{H}, P h \mathrm{CO}, J=7.3 \mathrm{~Hz}) ; 7.15\left(\mathrm{~d}, 2 \mathrm{H}, P h \mathrm{OCH}_{3}, J=\right.$ $8.6 \mathrm{~Hz}) ; 7.02\left(\mathrm{~d}, 2 \mathrm{H}, P_{h O C H}, J=8.6 \mathrm{~Hz}\right) ; 6.46\left(\mathrm{~s}, 1 \mathrm{H}, P h \mathrm{OCH}_{3}\right) ; 4.53\left(\mathrm{t}, 1 \mathrm{H}, \mathrm{CHCH}_{2}, J\right.$ $=4.4 \mathrm{~Hz}) ; 3.80\left(\mathrm{~s}, 3 \mathrm{H}, \mathrm{OCH}_{3}\right) ; 3.79\left(\mathrm{~s}, 3 \mathrm{H}, \mathrm{OCH}_{3}\right) ; 3.53\left(\mathrm{~s}, 3 \mathrm{H}, \mathrm{OCH}_{3}\right) ; 2.45(\mathrm{dd}, 1 \mathrm{H}$, $\mathrm{CHCH}_{2}, J=14.1$ and $\left.7.1 \mathrm{~Hz}\right) ; 1.97-2.10\left(\mathrm{~m}, 1 \mathrm{H}, \mathrm{CHCH}_{2}\right)$.

$3 \mathrm{i}^{1} \mathrm{H}$ NMR $\left(\mathrm{CDCl}_{3}\right) \delta 8.04(\mathrm{~d}, 2 \mathrm{H}, P h \mathrm{CO}, J=7.3 \mathrm{~Hz}) ; 7.46(\mathrm{t}, 2 \mathrm{H}, P h \mathrm{CO}, J=7.3 \mathrm{~Hz})$; $7.38(\mathrm{t}, 1 \mathrm{H}, P h \mathrm{CO}, J=7.3 \mathrm{~Hz}) ; 7.24\left(\mathrm{~d}, 2 \mathrm{H}, P h \mathrm{OCH}_{3}, J=8.6 \mathrm{~Hz}\right) ; 6.83\left(\mathrm{~d}, 2 \mathrm{H}, P h \mathrm{OCH}_{3}\right.$, $J=8.6 \mathrm{~Hz}) ; 6.33\left(\mathrm{~s}, 1 \mathrm{H}, P h \mathrm{OCH}_{3}\right) ; 4.99\left(\mathrm{dd}, 1 \mathrm{H}, \mathrm{CHCH}_{2}, J=10.1\right.$ and $\left.3.3 \mathrm{~Hz}\right) ; 4.18(\mathrm{dd}$, $1 \mathrm{H}, \mathrm{CHCH}_{2}, J=18.2$ and $10.4 \mathrm{~Hz}$ ); $3.85\left(\mathrm{dd}, 1 \mathrm{H}, \mathrm{CHCH}_{2}, J=15.9\right.$ and $4.0 \mathrm{~Hz}$ ); 3.77 (s, $\left.3 \mathrm{H}, \mathrm{OCH}_{3}\right) ; 3.76\left(\mathrm{~s}, 3 \mathrm{H}, \mathrm{OCH}_{3}\right) ; 3.66\left(\mathrm{~s}, 3 \mathrm{H}, \mathrm{OCH}_{3}\right) ; 3.23\left(\mathrm{~s}, 3 \mathrm{H}, \mathrm{OCH}_{3}\right)$.

Combined ${ }^{13} \mathrm{C}\left\{{ }^{1} \mathrm{H}\right\}$ NMR $\delta 201.5$ (C=O), 157.63 (Quat.), 157.57 (Quat.), 152.6 (Quat.), 152.1 (Quat.), 150.3 (Quat.), 148.9 (Quat.), 139.0 (Quat.), 136.6 (Quat.), 136.5 (Quat.), 135.8 (Quat.), 133.6 (Quat.), 128.6 (Arom. CH), 128.5 (Arom. CH), 128.42 (Arom. CH), 128.38 (Arom. CH), 128.3 (Arom. $\mathrm{CH}$ ), 128.2 (Arom. $\mathrm{CH}$ ), 125.1 (Arom. CH), 117.7 (Arom. CH), 113.6 (Arom. CH), 113.4 (Arom. CH), 108.4 (Arom. CH), $97.54(\mathrm{COH})$, $97.48(\mathrm{COH}), 60.8\left(\mathrm{OCH}_{3}\right), 60.6\left(\mathrm{OCH}_{3}\right), 60.5\left(\mathrm{OCH}_{3}\right), 59.8\left(\mathrm{OCH}_{3}\right), 59.4\left(\mathrm{OCH}_{3}\right), 55.8$ $\left(\mathrm{OCH}_{3}\right), 55.6\left(\mathrm{OCH}_{3}\right), 55.2\left(\mathrm{OCH}_{3}\right), 45.3\left(\mathrm{CHCH}_{2}\right), 41.6\left(\mathrm{CHCH}_{2}\right), 35.8\left(\mathrm{CHCH}_{2}\right), 34.1$ $\left(\mathrm{CHCH}_{2}\right) . v_{\max }(\mathrm{NaCl}) / \mathrm{cm}^{-1}: 3568,3340,3032,3003,1672,1609,1597,1581,1512$, 1487, 1448, 1248, 1197, 1180, 1128, 1036. HR-MS: $\mathrm{C}_{25} \mathrm{H}_{27} \mathrm{O}_{6} \mathrm{Na}$ Calcd. 445.1627 (M+Na); found: 445.1620. 

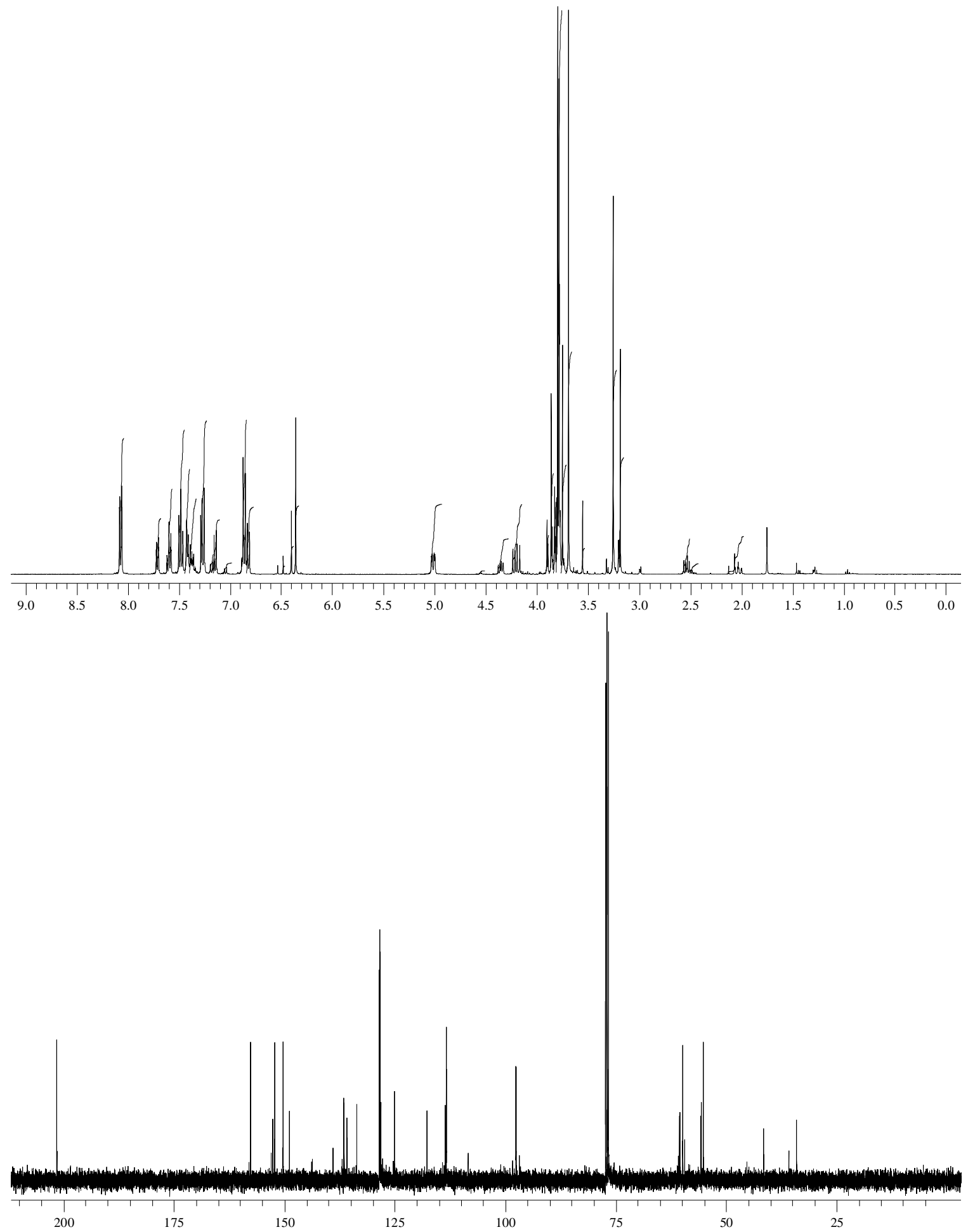

S-19 


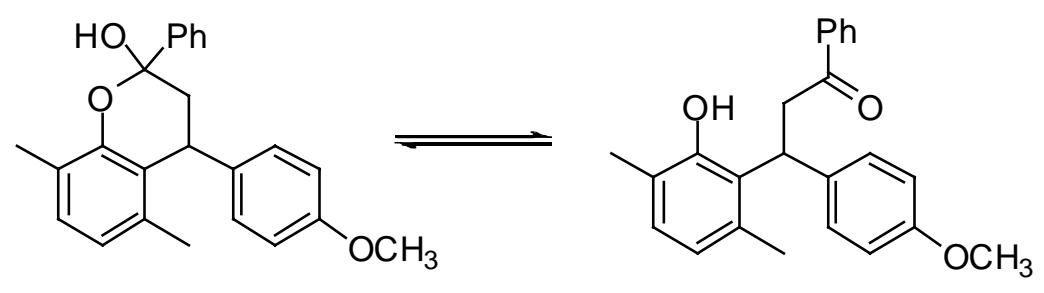

Column chromatography on silica gel (EtOAc/hexane $=1 / 4, \mathrm{R}_{\mathrm{f}}=0.3$ ) to afford $\mathbf{2} \mathbf{j} / \mathbf{3} \mathbf{j}$.

$\mathbf{2} \mathbf{j} / \mathbf{3} \mathbf{j}=13.5: 1, \mathbf{2} \mathbf{j} \mathrm{dr}=2.9: 1$

2j (major) ${ }^{1} \mathrm{H} \mathrm{NMR}\left(\mathrm{CDCl}_{3}\right) \delta 7.74$ (d, 2H, $\left.P h \mathrm{CO}, J=7.3 \mathrm{~Hz}\right) ; 7.42(\mathrm{t}, 2 \mathrm{H}, P h \mathrm{CO}, J=$ $7.3 \mathrm{~Hz}) ; 7.34-7.42(\mathrm{~m}, 1 \mathrm{H}, P h \mathrm{CO}) ; 7.05\left(\mathrm{~d}, 1 \mathrm{H}, P h \mathrm{CH}_{3}, J=7.6 \mathrm{~Hz}\right) ; 7.04(\mathrm{~d}, 2 \mathrm{H}$, $\left.P h \mathrm{OCH}_{3}, J=8.8 \mathrm{~Hz}\right) ; 6.79\left(\mathrm{~d}, 2 \mathrm{H}, P h \mathrm{OCH}_{3}, J=8.8 \mathrm{~Hz}\right) ; 6.71\left(\mathrm{~d}, 1 \mathrm{H}, P h \mathrm{CH}_{3}, J=7.6\right.$ $\mathrm{Hz}) ; 4.44\left(\mathrm{dd}, 1 \mathrm{H}, \mathrm{CHCH}_{2}, J=11.1\right.$ and $\left.7.3 \mathrm{~Hz}\right) ; 3.77\left(\mathrm{~s}, 3 \mathrm{H}, \mathrm{OCH}_{3}\right) ; 3.02(\mathrm{~d}, 1 \mathrm{H}, \mathrm{OH}, J$ $=2.3 \mathrm{~Hz}) ; 2.69\left(\mathrm{dd}, 1 \mathrm{H}, \mathrm{CHCH}_{2}, J=13.9\right.$ and $\left.7.3 \mathrm{~Hz}\right) ; 2.36\left(\mathrm{~s}, 3 \mathrm{H}, \mathrm{PhCH}_{3}\right) ; 2.12$ (ddd, $1 \mathrm{H}, \mathrm{CHCH}_{2}, J=13.9,11.4$ and $\left.2.5 \mathrm{~Hz}\right) ; 1.81$ (s, $\left.3 \mathrm{H}, \mathrm{PhCH}_{3}\right)$.

2j (minor) ${ }^{1} \mathrm{H}$ NMR $\left(\mathrm{CDCl}_{3}\right) \delta 7.65$ (d, 2H, PhCO, $\left.J=7.3 \mathrm{~Hz}\right) ; 7.48$ (t, $2 \mathrm{H}, P h \mathrm{CO}, J=$ $7.3 \mathrm{~Hz}) ; 7.34-7.42(\mathrm{~m}, 1 \mathrm{H}, P h \mathrm{CO}) ; 7.15\left(\mathrm{~d}, 2 \mathrm{H}, P h \mathrm{OCH}_{3}, J=8.8 \mathrm{~Hz}\right) ; 7.14(\mathrm{~d}, 1 \mathrm{H}$, $\left.P h \mathrm{CH}_{3}, J=7.6 \mathrm{~Hz}\right) ; 6.88\left(\mathrm{~d}, 2 \mathrm{H}, P h \mathrm{OCH}_{3}, J=8.8 \mathrm{~Hz}\right) ; 6.82\left(\mathrm{~d}, 1 \mathrm{H}, P h \mathrm{CH}_{3}, J=7.6 \mathrm{~Hz}\right)$; $4.31\left(\mathrm{~d}, \mathrm{br}, 1 \mathrm{H}, \mathrm{CHCH}_{2}, J=5.3 \mathrm{~Hz}\right) ; 3.81\left(\mathrm{~s}, 3 \mathrm{H}, \mathrm{OCH}_{3}\right) ; 3.19(\mathrm{~s}, 1 \mathrm{H}, \mathrm{OH}) ; 2.66-2.71(\mathrm{~m}$, $\left.1 \mathrm{H}, \mathrm{CHCH}_{2}\right) ; 2.48\left(\mathrm{dd}, 1 \mathrm{H}, \mathrm{CHCH}_{2}, J=14.1\right.$ and $\left.6.8 \mathrm{~Hz}\right) ; 2.39$ (s, $\left.3 \mathrm{H}, \mathrm{PhCH}_{3}\right) ; 2.01$ (s, $\left.3 \mathrm{H}, \mathrm{PhCH}_{3}\right)$.

$3 \mathbf{j}^{1} \mathrm{H}$ NMR $\left(\mathrm{CDCl}_{3}\right) \delta 8.03(\mathrm{~d}, 2 \mathrm{H}, P h \mathrm{CO}, J=7.3 \mathrm{~Hz}) ; 7.59(\mathrm{t}, 2 \mathrm{H}, P h \mathrm{CO}, J=7.3 \mathrm{~Hz})$; 7.34-7.42 (m, 1H, $P h \mathrm{CO}) ; 7.20$ (d, $\left.2 \mathrm{H}, P h \mathrm{OCH}_{3}, J=8.8 \mathrm{~Hz}\right) ; 6.93\left(\mathrm{~d}, 1 \mathrm{H}, P h \mathrm{CH}_{3}, J=\right.$ $7.6 \mathrm{~Hz}) ; 6.85\left(\mathrm{~d}, 2 \mathrm{H}, P h \mathrm{OCH}_{3}, J=8.8 \mathrm{~Hz}\right) ; 5.26\left(\mathrm{t}, 1 \mathrm{H}, \mathrm{CHCH}_{2}, J=6.8 \mathrm{~Hz}\right) ; 4.02$ (dd, $1 \mathrm{H}, \mathrm{CHCH}_{2}, J=17.4$ and $\left.6.6 \mathrm{~Hz}\right) ; 3.85-3.95\left(\mathrm{~m}, 1 \mathrm{H}, \mathrm{CHCH}_{2}\right) ; 3.79\left(\mathrm{~s}, 3 \mathrm{H}, \mathrm{OCH}_{3}\right) ; 2.20$ (s, $3 \mathrm{H}, \mathrm{PhCH}_{3}$ ).

Combined ${ }^{13} \mathrm{C}\left\{{ }^{1} \mathrm{H}\right\}$ NMR $\delta 158.1$ (Quat.), 157.6 (Quat.), 151.3 (Quat.), 151.0 (Quat.), 144.5 (Quat.), 144.0 (Quat.), 138.0 (Quat.), 135.8 (Quat.), 135.6 (Quat.), 135.5 (Quat.), 129.1 (Arom. CH), 128.8 (Arom. CH), 128.6 (Arom. CH), 128.5 (Arom. $\mathrm{CH}$ ), 128.4 (Arom. $\mathrm{CH}$ ), 128.33 (Arom. CH), 128.25 (Arom. CH), 128.16 (Arom. CH), 128.14 (Arom. CH), 128.10 (Arom. CH), 125.4 (Arom. $\mathrm{CH}$ ), 125.2 (Arom. $\mathrm{CH}$ ), 124.20 (Quat.), 124.16 (Quat.), 123.31 (Arom. CH), 123.28 (Arom. CH), 122.2 (Quat.), 120.2 (Quat.), 114.1 (Arom. CH), 113.9 (Arom. $\mathrm{CH}$ ), $97.7(\mathrm{COH}), 96.7(\mathrm{COH}), 55.13\left(\mathrm{OCH}_{3}\right), 55.08$ $\left(\mathrm{OCH}_{3}\right), 46.2\left(\mathrm{CHCH}_{2}\right), 42.2\left(\mathrm{CHCH}_{2}\right), 38.2\left(\mathrm{CHCH}_{2}\right), 36.2\left(\mathrm{CHCH}_{2}\right), 21.2\left(\mathrm{ArCH}_{3}\right)$, $20.6\left(\mathrm{ArCH}_{3}\right), 19.0\left(\mathrm{ArCH}_{3}\right), 16.4\left(\mathrm{ArCH}_{3}\right), 16.3\left(\mathrm{ArCH}_{3}\right) \cdot v_{\max }(\mathrm{NaCl}) / \mathrm{cm}^{-1}: 3562,3032$, 3003, 1608, 1583, 1510, 1461, 1448, 1411, 1255, 1245, 1213, 1176, 1054, 1033, 1014. HR-MS: $\mathrm{C}_{24} \mathrm{H}_{25} \mathrm{O}{ }_{3}$ Calcd. $361.1804(\mathrm{M}+\mathrm{H})$; found: 361.1801 . 


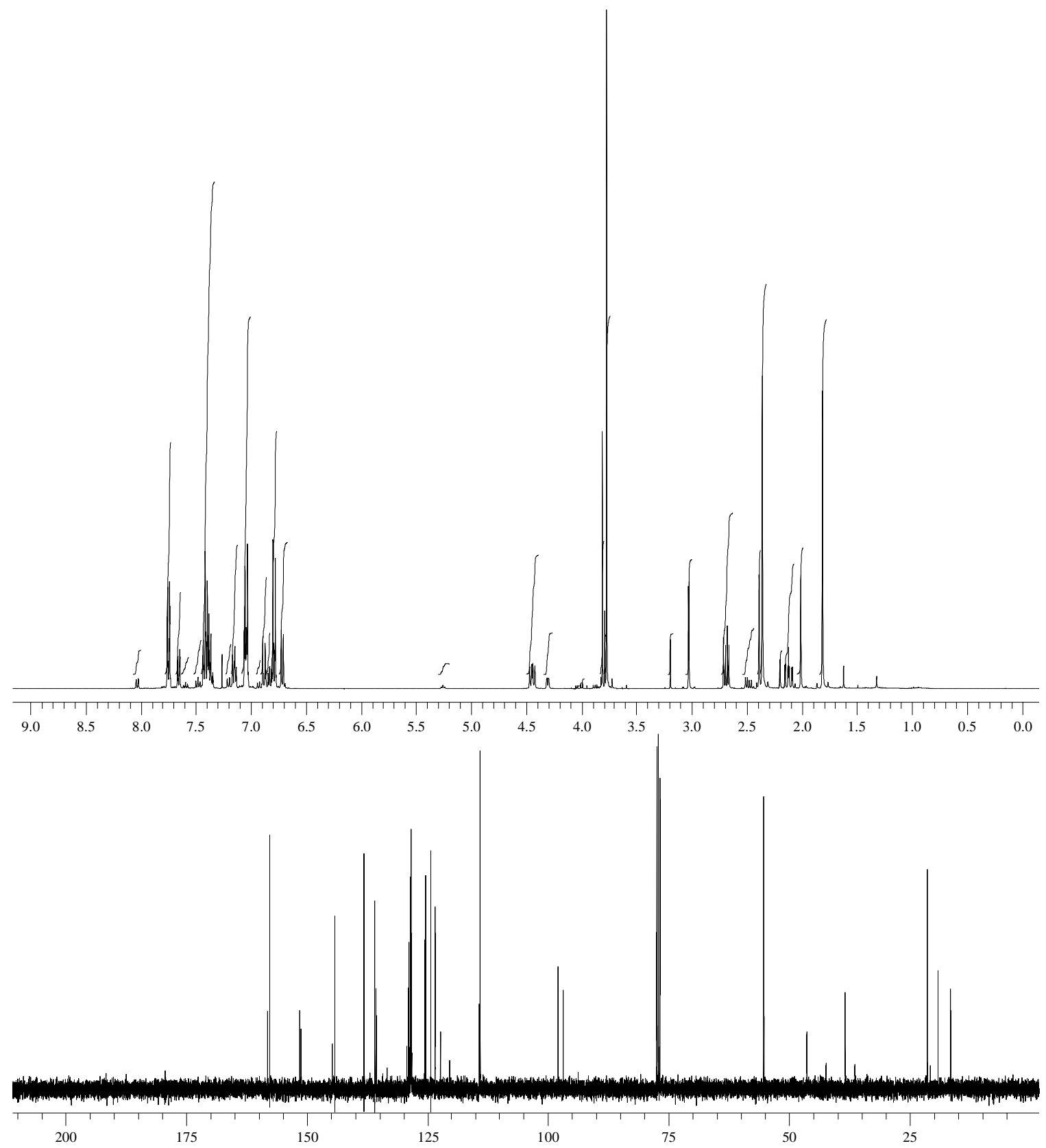

S-21 


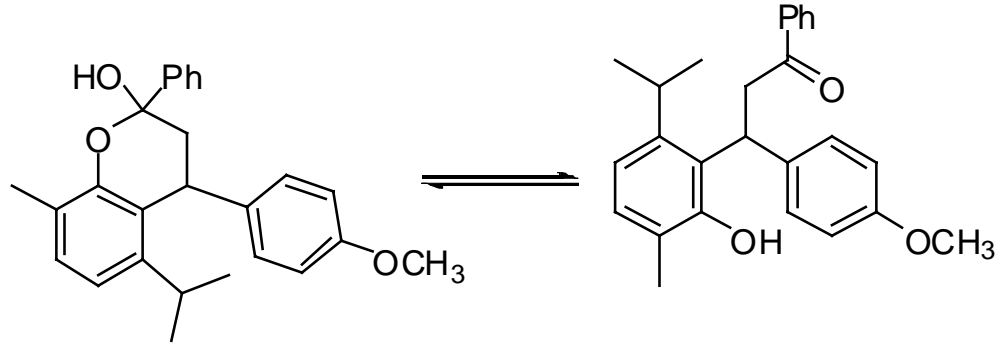

Column chromatography on silica gel $\left(\right.$ EtOAc/hexane $\left.=1 / 4, R_{\mathrm{f}}=0.3\right)$ to afford $\mathbf{2 k} / \mathbf{3} \mathbf{k}$.

$\mathbf{2} \mathbf{k} / \mathbf{3} \mathbf{k}=15.7: 1, \mathbf{2} \mathbf{k} \mathrm{dr}=2: 1$

2k (major) ${ }^{1} \mathrm{H}$ NMR $\left(\mathrm{CDCl}_{3}\right) \delta 7.70(\mathrm{~d}, 2 \mathrm{H}, P h \mathrm{CO}, J=8.1 \mathrm{~Hz}) ; 7.37-7.41(\mathrm{~m}, 2 \mathrm{H}$, $P h \mathrm{CO}) ; 7.35-7.39(\mathrm{~m}, 1 \mathrm{H}, P h \mathrm{CO}) ; 7.16\left(\mathrm{~d}, 1 \mathrm{H}, P h \mathrm{CH}_{3}, J=7.6 \mathrm{~Hz}\right) ; 7.00(\mathrm{~d}, 2 \mathrm{H}$, $\left.P h \mathrm{OCH}_{3}, J=8.8 \mathrm{~Hz}\right) ; 6.98\left(\mathrm{~d}, 1 \mathrm{H}, P h \mathrm{CH}_{3}, J=8.0 \mathrm{~Hz}\right) ; 6.76\left(\mathrm{~d}, 2 \mathrm{H}, P h \mathrm{OCH}_{3}, J=8.8\right.$ $\mathrm{Hz}) ; 4.56\left(\mathrm{dd}, 1 \mathrm{H}, \mathrm{CHCH} \mathrm{CH}_{2}, J=10.4\right.$ and $\left.7.6 \mathrm{~Hz}\right) ; 3.77\left(\mathrm{~s}, 3 \mathrm{H}, \mathrm{OCH}_{3}\right) ; 2.97(\mathrm{~d}, 1 \mathrm{H}, \mathrm{OH}, J$ $=2.3 \mathrm{~Hz}) ; 2.84-2.90\left(\mathrm{~m}, 1 \mathrm{H}, \mathrm{CH}\left(\mathrm{CH}_{3}\right)_{2}\right) ; 2.73\left(\mathrm{dd}, 1 \mathrm{H}, \mathrm{CHCH}_{2}, J=14.1\right.$ and $\left.2.0 \mathrm{~Hz}\right)$; $2.37\left(\mathrm{~s}, 3 \mathrm{H}, \mathrm{PhCH}_{3}\right) ; 2.17\left(\mathrm{ddd}, 1 \mathrm{H}, \mathrm{CHCH}_{2}, J=13.9,10.4\right.$ and $\left.2.3 \mathrm{~Hz}\right) ; 1.11(\mathrm{~d}, 1 \mathrm{H}$, $\left.\mathrm{CH}\left(\mathrm{CH}_{3}\right)_{2}, J=6.8 \mathrm{~Hz}\right) ; 0.72\left(\mathrm{~d}, 1 \mathrm{H}, \mathrm{CH}\left(\mathrm{CH}_{3}\right)_{2}, J=6.8 \mathrm{~Hz}\right)$.

2k (minor) ${ }^{1} \mathrm{H}$ NMR $\left(\mathrm{CDCl}_{3}\right) \delta 7.66(\mathrm{~d}, 2 \mathrm{H}, P h \mathrm{CO}, J=8.1 \mathrm{~Hz}) ; 7.39-7.43(\mathrm{~m}, 2 \mathrm{H}$, $P h \mathrm{CO}) ; 7.32-7.35(\mathrm{~m}, 1 \mathrm{H}, P h \mathrm{CO}) ; 7.25\left(\mathrm{~d}, 1 \mathrm{H}, P h \mathrm{CH}_{3}, J=8.0 \mathrm{~Hz}\right) ; 7.18(\mathrm{~d}, 2 \mathrm{H}$, $\left.P h \mathrm{OCH}_{3}, J=8.8 \mathrm{~Hz}\right) ; 6.90\left(\mathrm{~d}, 2 \mathrm{H}, P h \mathrm{OCH}_{3}, J=8.8 \mathrm{~Hz}\right) ; 6.89\left(\mathrm{~d}, 1 \mathrm{H}, P h \mathrm{CH}_{3}, J=8.0\right.$ $\mathrm{Hz}$ ); $4.48\left(\mathrm{~d}, \mathrm{br}, 1 \mathrm{H}, \mathrm{CHCH}_{2}, J=5.3 \mathrm{~Hz}\right) ; 3.82\left(\mathrm{~s}, 3 \mathrm{H}, \mathrm{OCH}_{3}\right) ; 3.17$ (s, $\left.1 \mathrm{H}, \mathrm{OH}\right) ; 2.74-$ $2.81\left(\mathrm{~m}, 1 \mathrm{H}, \mathrm{CH}\left(\mathrm{CH}_{3}\right)_{2}\right) ; 2.72$ (dd, $1 \mathrm{H}, \mathrm{CHCH}_{2}, J=13.9$ and $\left.7.6 \mathrm{~Hz}\right) ; 2.48$ (dd, $1 \mathrm{H}$, $\mathrm{CHCH}_{2}, J=14.2$ and $\left.6.8 \mathrm{~Hz}\right) ; 2.39\left(\mathrm{~s}, 3 \mathrm{H}, \mathrm{PhCH}_{3}\right) ; 1.17\left(\mathrm{~d}, 1 \mathrm{H}, \mathrm{CH}\left(\mathrm{CH}_{3}\right)_{2}, J=6.8 \mathrm{~Hz}\right)$; $1.02\left(\mathrm{~d}, 1 \mathrm{H}, \mathrm{CH}\left(\mathrm{CH}_{3}\right)_{2}, J=6.8 \mathrm{~Hz}\right)$.

3k ${ }^{1} \mathrm{H}$ NMR $\left(\mathrm{CDCl}_{3}\right) \delta 8.07(\mathrm{~d}, 2 \mathrm{H}, P h \mathrm{CO}, J=8.1 \mathrm{~Hz}) ; 7.50(\mathrm{t}, 2 \mathrm{H}, P h \mathrm{CO}, J=8.1 \mathrm{~Hz})$; $7.06\left(\mathrm{~d}, 1 \mathrm{H}, P h \mathrm{CH}_{3}, J=8.0 \mathrm{~Hz}\right) ; 6.85\left(\mathrm{~d}, 2 \mathrm{H}, P h \mathrm{OCH}_{3}, J=8.8 \mathrm{~Hz}\right) ; 5.44\left(\mathrm{t}, 1 \mathrm{H}, \mathrm{CHCH}_{2}\right.$, $J=6.8 \mathrm{~Hz}) ; 3.79\left(\mathrm{~s}, 3 \mathrm{H}, \mathrm{OCH}_{3}\right)$.

Combined ${ }^{13} \mathrm{C}\left\{{ }^{1} \mathrm{H}\right\}$ NMR $\delta 158.2$ (Quat.), 157.6 (Quat.), 151.0 (Quat.), 150.8 (Quat.), 146.6 (Quat.), 146.1 (Quat.), 144.6 (Quat.), 144.0 (Quat.), 139.1 (Quat.), 136.2 (Quat.), 129.7 (Arom. CH), 129.1 (Arom. CH), 128.8 (Arom. CH), 128.34 (Arom. CH), 128.26 (Arom. CH), 128.2 (Arom. CH), 128.09 (Arom. CH), 128.07 (Arom. CH), 128.04 (Arom. $\mathrm{CH}$ ), 125.4 (Arom. CH), 125.2 (Arom. CH), 123.9 (Quat.), 123.7 (Quat.), 122.3 (Quat.), 118.8 (Arom. CH), 118.7 (Arom. $\mathrm{CH}$ ), 117.6 (Arom. $\mathrm{CH}$ ), 114.1 (Arom. $\mathrm{CH}$ ), 113.8 (Arom. $\mathrm{CH}), 97.6(\mathrm{COH}), 96.6(\mathrm{COH}), 55.2\left(\mathrm{OCH}_{3}\right), 55.1\left(\mathrm{OCH}_{3}\right), 46.3\left(\mathrm{CHCH}_{2}\right), 42.3$ $\left(\mathrm{CHCH}_{2}\right), 37.3\left(\mathrm{CHCH}_{2}\right), 35.3\left(\mathrm{CHCH}_{2}\right), 29.1\left(\mathrm{CH}\left(\mathrm{CH}_{3}\right)_{2}\right), 28.3\left(\mathrm{CH}\left(\mathrm{CH}_{3}\right)_{2}\right), 25.1$ $\left(\mathrm{CH}\left(\mathrm{CH}_{3}\right)_{2}\right), 25.0\left(\mathrm{CH}\left(\mathrm{CH}_{3}\right)_{2}\right), 22.8\left(\mathrm{CH}\left(\mathrm{CH}_{3}\right)_{2}\right), 22.2\left(\mathrm{CH}\left(\mathrm{CH}_{3}\right)_{2}\right), 16.5\left(\mathrm{ArCH}_{3}\right), 16.3$ $\left(\mathrm{ArCH}_{3}\right) . v_{\max }(\mathrm{NaCl}) / \mathrm{cm}^{-1}: 3560,3032,3005,1683,1608,1581,1461,1485,1448,1413$, 1203, 1176, 1033, 1014. HR-MS: $\mathrm{C}_{26} \mathrm{H}_{28} \mathrm{O}_{3} \mathrm{Na}$ Calcd. $411.1936(\mathrm{M}+\mathrm{Na})$; found: 411.1930 . 

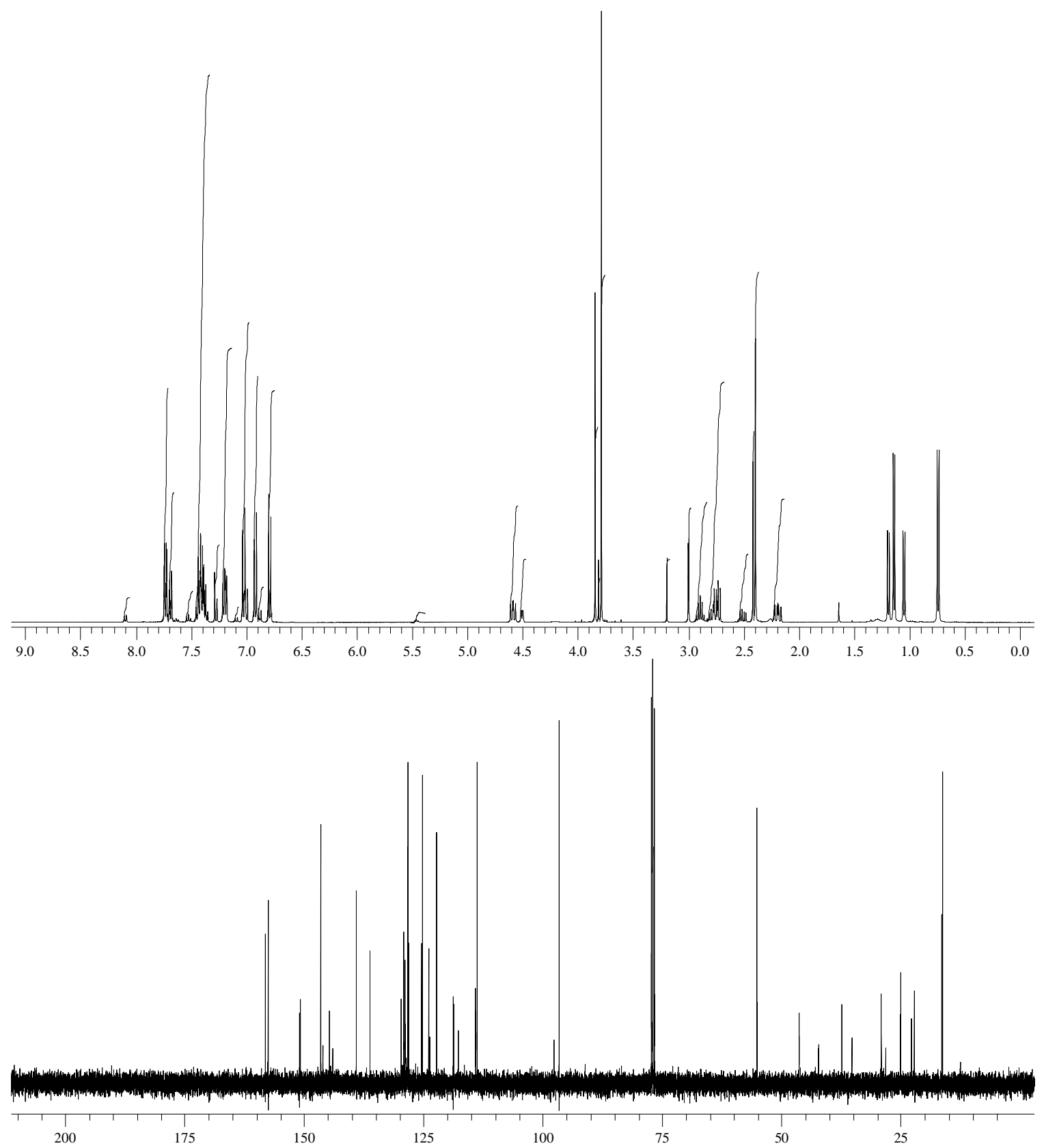

S-23 


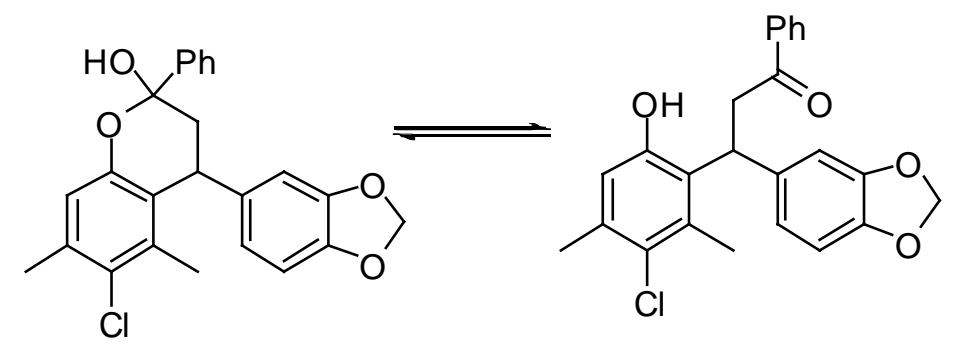

Column chromatography on silica gel (EtOAc/hexane $\left.=1 / 4, R_{\mathrm{f}}=0.2\right)$ to afford $2 \mathbf{l} / \mathbf{3 1}$.

$2 \mathbf{l} / 3 \mathbf{l}=6.8: 1,2 \mathrm{ldr}=2.2: 1$

$2 \mathrm{l}$ (major) ${ }^{1} \mathrm{H}$ NMR $\left(\mathrm{CDCl}_{3}\right) \delta 7.64(\mathrm{~d}, 2 \mathrm{H}, P h \mathrm{CO}, J=7.3 \mathrm{~Hz}) ; 7.37(\mathrm{t}, 2 \mathrm{H}, P h \mathrm{CO}, J=$ $7.3 \mathrm{~Hz}$ ); 7.33-7.41 (m, 1H, PhCO); 6.82 (s, 1H, PhCl); 6.68 (d, $1 \mathrm{H}, P h \mathrm{OCH}_{2} \mathrm{O}, J=8.0$

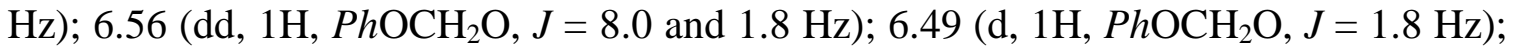
5.87-5.89 (m, 2H, $\left.\mathrm{OCH}_{2} \mathrm{O}\right) ; 4.38\left(\mathrm{dd}, 1 \mathrm{H}, \mathrm{CHCH}_{2}, J=10.7\right.$ and $\left.7.3 \mathrm{~Hz}\right) ; 3.20(\mathrm{~d}, 1 \mathrm{H}$, $\mathrm{OH}, J=2.5 \mathrm{~Hz}) ; 2.61\left(\mathrm{dd}, 1 \mathrm{H}, \mathrm{CHCH}_{2}, J=14.1\right.$ and $\left.7.6 \mathrm{~Hz}\right) ; 2.42-2.48(\mathrm{~m}, 1 \mathrm{H}$, $\left.\mathrm{CHCH}_{2}\right) ; 2.38\left(\mathrm{~s}, 3 \mathrm{H}, \mathrm{CH}_{3}\right) ; 2.01-2.06\left(\mathrm{~m}, 1 \mathrm{H}, \mathrm{CHCH}_{2}\right) ; 1.96\left(\mathrm{~s}, 3 \mathrm{H}, \mathrm{CH}_{3}\right)$.

$2 \mathrm{l}$ (minor) ${ }^{1} \mathrm{H}$ NMR $\left(\mathrm{CDCl}_{3}\right) \delta 7.58(\mathrm{~d}, 2 \mathrm{H}, P h \mathrm{CO}, J=7.3 \mathrm{~Hz}) ; 7.33-7.41(\mathrm{~m}, 2 \mathrm{H}$, $P h \mathrm{CO}) ; 7.33-7.41$ (m, $1 \mathrm{H}, P h \mathrm{CO}) ; 6.91(\mathrm{~s}, 1 \mathrm{H}, \mathrm{PhCl}) ; 6.74\left(\mathrm{~d}, 1 \mathrm{H}, P h \mathrm{OCH}_{2} \mathrm{O}, J=8.0\right.$ $\mathrm{Hz}) ; 6.70\left(\mathrm{~d}, 1 \mathrm{H}, P h \mathrm{OCH}_{2} \mathrm{O}, J=1.8 \mathrm{~Hz}\right) ; 6.61\left(\mathrm{dd}, 1 \mathrm{H}, P h \mathrm{OCH}_{2} \mathrm{O}, J=8.0\right.$ and $\left.1.8 \mathrm{~Hz}\right)$; $5.94\left(\mathrm{~s}, 2 \mathrm{H}, \mathrm{OCH}_{2} \mathrm{O}\right) ; 4.25$ (d, br, $\left.1 \mathrm{H}, \mathrm{CHCH}_{2}, J=6.6 \mathrm{~Hz}\right) ; 3.25$ (s, $\left.1 \mathrm{H}, \mathrm{OH}\right) ; 2.60$ (dd, $1 \mathrm{H}, \mathrm{CHCH}_{2}, J=14.4$ and $\left.5.3 \mathrm{~Hz}\right) ; 2.43\left(\mathrm{~s}, 3 \mathrm{H}, \mathrm{CH}_{3}\right) ; 2.09\left(\mathrm{~s}, 3 \mathrm{H}, \mathrm{CH}_{3}\right)$.

$31{ }^{1} \mathrm{H}$ NMR $\left(\mathrm{CDCl}_{3}\right) \delta 7.98(\mathrm{~d}, 2 \mathrm{H}, P h \mathrm{CO}, J=7.3 \mathrm{~Hz}) ; 7.46(\mathrm{t}, 2 \mathrm{H}, P h \mathrm{CO}, J=7.3 \mathrm{~Hz})$; 7.33-7.41 (m, 1H, PhCO); $6.72(\mathrm{~s}, 1 \mathrm{H}, \mathrm{PhCl}) ; 5.87-5.89\left(\mathrm{~m}, 2 \mathrm{H}, \mathrm{OCH}_{2} \mathrm{O}\right) ; 5.20(\mathrm{t}, 1 \mathrm{H}$, $\left.\mathrm{CHCH}_{2}, J=6.9 \mathrm{~Hz}\right) ; 3.95\left(\mathrm{dd}, 1 \mathrm{H}, \mathrm{CHCH}_{2}, J=17.7\right.$ and $\left.8.1 \mathrm{~Hz}\right) ; 3.86\left(\mathrm{dd}, 1 \mathrm{H}, \mathrm{CHCH}_{2}\right.$, $J=17.7$ and $5.8 \mathrm{~Hz}) ; 2.28\left(\mathrm{~s}, 3 \mathrm{H}, \mathrm{CH}_{3}\right)$.

Combined ${ }^{13} \mathrm{C}\left\{{ }^{1} \mathrm{H}\right\}$ NMR $\delta 152.3$ (Quat.), 151.3 (Quat.), 151.2 (Quat.), 148.0 (Quat.), 147.8 (Quat.), 147.7 (Quat.), 146.2 (Quat.), 145.8 (Quat.), 145.6 (Quat.), 143.8 (Quat.), 143.3 (Quat.), 139.6 (Quat.), 137.6 (Quat.), 136.41 (Quat.), 136.37 (Quat.), 136.0 (Quat.), 135.8 (Quat.), 135.7 (Quat.), 133.4 (Quat.), 128.6 (Arom. CH), 128.5 (Arom. CH), 128.32 (Arom. CH), 128.27 (Arom. CH), 128.2 (Arom. CH), 128.16 (Arom. CH), 127.5 (Arom. CH), 125.3 (Arom. CH), 125.1 (Arom. CH), 122.7 (Quat.), 120.5 (Arom. CH), 120.3 (Arom. CH), 119.5 (Quat.), 117.6 (Arom. CH), 117.5 (Arom. CH), 108.40 (Arom. $\mathrm{CH}$ ), 108.38 (Arom. $\mathrm{CH}$ ), 108.35 (Arom. $\mathrm{CH}$ ), 108.1 (Arom. $\mathrm{CH}$ ), 108.0 (Arom. $\mathrm{CH}$ ), 107.5 (Arom. $\mathrm{CH}), 101.0$ (Arom. $\mathrm{CH}), 100.9\left(\mathrm{OCH}_{2} \mathrm{O}\right), 100.7\left(\mathrm{OCH}_{2} \mathrm{O}\right), 97.7(\mathrm{COH})$, $97.0(\mathrm{COH}), 46.2\left(\mathrm{CHCH}_{2}\right), 42.3\left(\mathrm{CHCH}_{2}\right), 39.1\left(\mathrm{CHCH}_{2}\right), 37.4\left(\mathrm{CHCH}_{2}\right), 20.9$ $\left(\mathrm{ArCH}_{3}\right), 20.84\left(\mathrm{ArCH}_{3}\right), 20.82\left(\mathrm{ArCH}_{3}\right), 18.8\left(\mathrm{ArCH}_{3}\right), 17.7\left(\mathrm{ArCH}_{3}\right), 16.9\left(\mathrm{ArCH}_{3}\right)$. $v_{\max }(\mathrm{NaCl}) / \mathrm{cm}^{-1}:$ 3562, 3032, 3012, 1683, 1606, 1568, 1502, 1487, 1440, 1244, 1166 , 1068, 1039. HR-MS: $\mathrm{C}_{24} \mathrm{H}_{21} \mathrm{ClO}_{4}$ Calcd. 408.1128 (M+); found: 408.1127. 

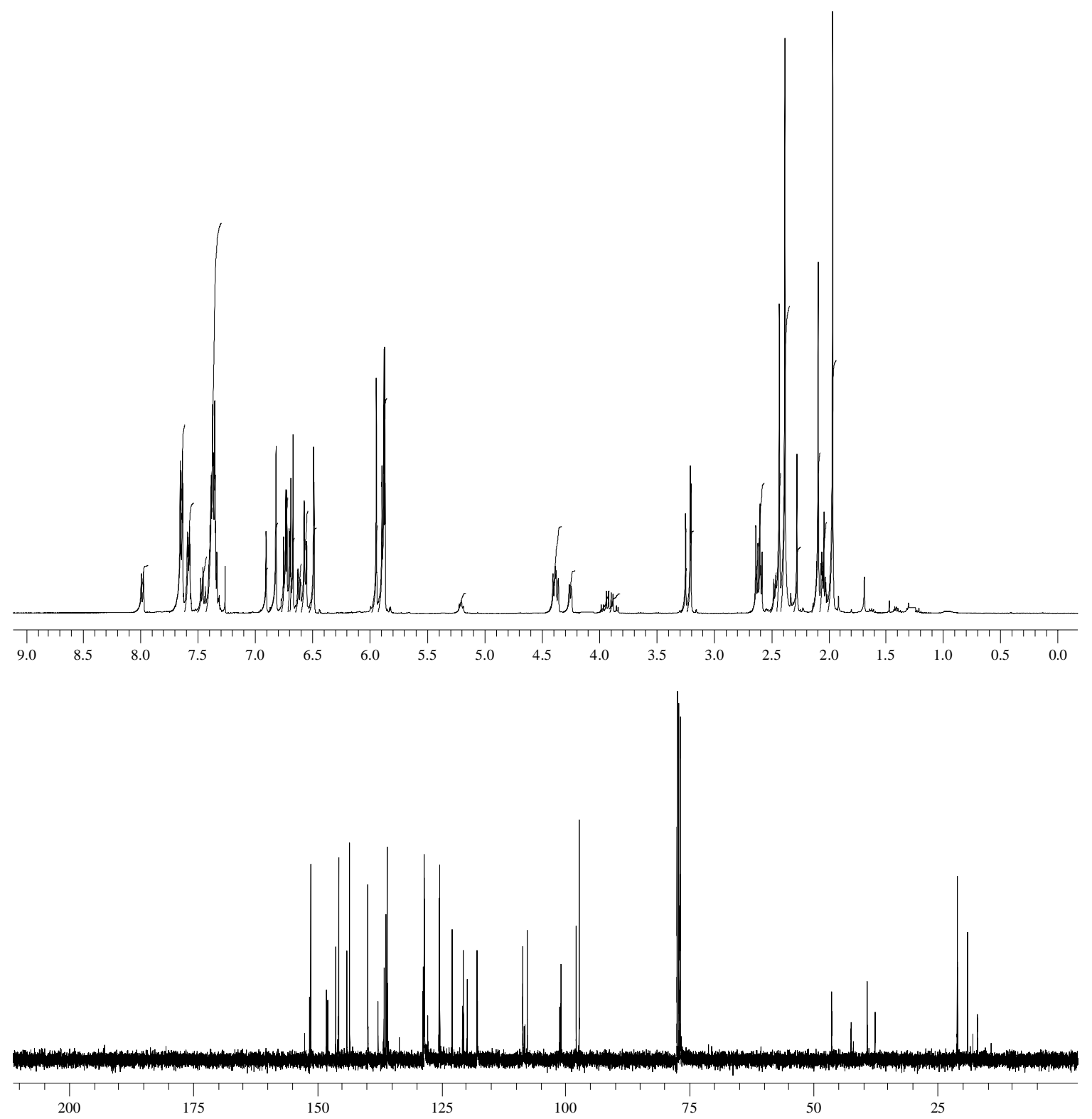

S-25 
$( \pm)$-cis-4a

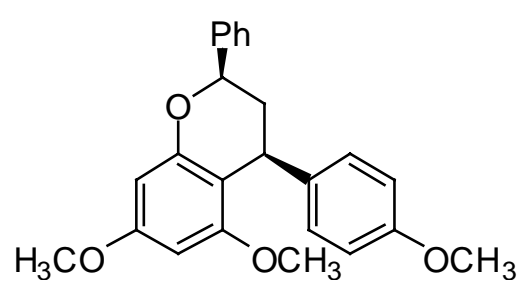

Column chromatography on silica gel $\left(\mathrm{DCM}, \mathrm{R}_{\mathrm{f}}=0.8\right)$ to afford $4 \mathbf{a}$.

(士)-cis-5,7-dimethoxy-4-(4-methoxyphenyl)-2-phenylchroman. ${ }^{1} \mathrm{H}$ NMR $\left(\mathrm{CDCl}_{3}\right) \delta 7.45$ $(\mathrm{d}, 2 \mathrm{H}, P h \mathrm{CHO}, J=7.1 \mathrm{~Hz}) ; 7.37(\mathrm{t}, 2 \mathrm{H}, P h \mathrm{CHO}, J=7.1 \mathrm{~Hz}) ; 7.31(\mathrm{t}, 1 \mathrm{H}, P h \mathrm{CHO}, J=$ $7.1 \mathrm{~Hz}) ; 7.03\left(\mathrm{~d}, 2 \mathrm{H}, P h \mathrm{OCH}_{3}, J=8.8 \mathrm{~Hz}\right) ; 6.77\left(\mathrm{~d}, 2 \mathrm{H}, P h \mathrm{OCH}_{3}, J=8.8 \mathrm{~Hz}\right) ; 6.23(\mathrm{~d}$, $\left.1 \mathrm{H}, P h \mathrm{OCH}_{3}, J=2.4 \mathrm{~Hz}\right) ; 6.06\left(\mathrm{~d}, 1 \mathrm{H}, \mathrm{PhOCH}_{3}, J=2.4 \mathrm{~Hz}\right) ; 4.99$ (dd, $1 \mathrm{H}, \mathrm{PhCHO}, J=$ 11.4 and $1.4 \mathrm{~Hz}) ; 4.24\left(\mathrm{dd}, 1 \mathrm{H}, \mathrm{CH}_{3} \mathrm{OPhCH}, J=11.1\right.$ and $\left.7.6 \mathrm{~Hz}\right) ; 3.79\left(\mathrm{~s}, 3 \mathrm{H}, \mathrm{OCH}_{3}\right)$; $3.78\left(\mathrm{~s}, 3 \mathrm{H}, \mathrm{OCH}_{3}\right) ; 3.40\left(\mathrm{~s}, 3 \mathrm{H}, \mathrm{OCH}_{3}\right) ; 2.50\left(\mathrm{ddd}, 1 \mathrm{H}, \mathrm{CHCH}_{2}, J=14.0,7.6\right.$ and 1.5 $\mathrm{Hz}$ ); 2.05-2.14 (m, 1H, CHCH$\left.{ }_{2}\right) .{ }^{13} \mathrm{C}\left\{{ }^{1} \mathrm{H}\right\} \mathrm{NMR} \delta 159.7$ (Quat.), 159.5 (Quat.), 158.0 (Quat.), 157.3 (Quat.), 140.8 (Quat.), 139.5 (Quat.), 128.5 (Arom. CH), 127.9 (Arom. $\mathrm{CH}$ ), 127.4 (Arom. CH), 126.2 (Arom. $\mathrm{CH}$ ), 113.3 (Arom. $\mathrm{CH}$ ), 107.5 (Quat.), 93.7 (Arom. CH), 93.1 (Arom. $\mathrm{CH}), 78.0(\mathrm{CH}-\mathrm{O}), 55.3\left(\mathrm{OCH}_{3}\right), 55.2\left(\mathrm{OCH}_{3}\right), 55.1\left(\mathrm{OCH}_{3}\right)$, $42.6\left(\mathrm{ArCHCH}_{2}\right), 38.4(\mathrm{ArCHCH}) . v_{\max }(\mathrm{NaCl}) / \mathrm{cm}^{-1}: 3032,3005,1614,1589,1510$, 1492, 1465, 1248, 1203, 1147, 1112, 1036. HR-MS: $\mathrm{C}_{24} \mathrm{H}_{25} \mathrm{O}_{4}$ Calcd. $377.1753(\mathrm{M}+\mathrm{H})$; found: 377.1745 .

$( \pm)-$ trans-4a

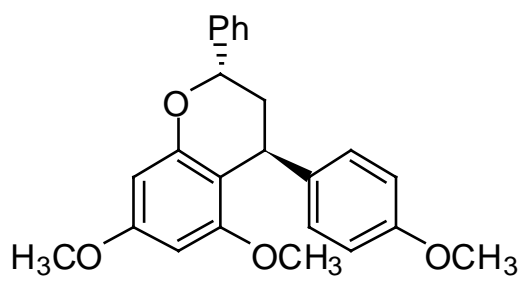

(士)-trans-5,7-dimethoxy-4-(4-methoxyphenyl)-2-phenylchroman. ${ }^{1} \mathrm{H}$ NMR $\left(\mathrm{CDCl}_{3}\right) \delta$ 7.28-7.36 (m, 5H, $P h \mathrm{CH}) ; 7.08\left(\mathrm{~d}, 2 \mathrm{H}, P h \mathrm{OCH}_{3}, J=8.7 \mathrm{~Hz}\right) ; 6.86\left(\mathrm{~d}, 2 \mathrm{H}, P h \mathrm{OCH}_{3}, J=\right.$ $8.7 \mathrm{~Hz}) ; 6.27\left(\mathrm{~d}, 1 \mathrm{H}, P h \mathrm{OCH}_{3}, J=2.3 \mathrm{~Hz}\right) ; 6.15\left(\mathrm{~d}, 1 \mathrm{H}, P h \mathrm{OCH}_{3}, J=2.3 \mathrm{~Hz}\right) ; 4.89$ (dd, $1 \mathrm{H}, \mathrm{PhCHO}, J=12.0$ and 2.0 Hz); $4.36\left(\mathrm{~d}, \mathrm{br}, 1 \mathrm{H}, \mathrm{CH}_{3} \mathrm{OPhCH}, J=4.1 \mathrm{~Hz}\right) ; 3.83$ (s, 3H, $\left.\mathrm{OCH}_{3}\right) ; 3.82\left(\mathrm{~s}, 3 \mathrm{H}, \mathrm{OCH}_{3}\right) ; 3.66\left(\mathrm{~s}, 3 \mathrm{H}, \mathrm{OCH}_{3}\right) ; 2.29-2.37\left(\mathrm{~m}, 1 \mathrm{H}, \mathrm{CHCH}_{2}\right) ; 2.10-1.18$ (m, 1H, $\left.\mathrm{CHCH}_{2}\right) .{ }^{13} \mathrm{C}\left\{{ }^{1} \mathrm{H}\right\}$ NMR $\delta 160.0$ (Quat.), 158.7 (Quat.), 157.7 (Quat.), 156.6 (Quat.), 141.4 (Quat.), 138.2 (Quat.), 128.8 (Arom. CH), 128.3 (Arom. CH), 127.8 (Arom. CH), 127.4 (Arom. CH), 126.2 (Arom. CH), 104.5 (Quat.), 93.0 (Arom. CH), 91.7 (Arom. $\mathrm{CH}), 73.0(\mathrm{CH}-\mathrm{O}), 55.5\left(\mathrm{OCH}_{3}\right), 55.2\left(\mathrm{OCH}_{3}\right), 55.1\left(\mathrm{OCH}_{3}\right), 38.5$ $(\mathrm{ArCHCH}), 34.4\left(\mathrm{ArCHCH}_{2}\right) . v_{\max }(\mathrm{NaCl}) / \mathrm{cm}^{-1}$ : 3031, 3005, 1616, 1593, 1510, 1495 , 1465, 1456, 1246, 1205, 1195, 1147, 1113, 1034. HR-MS: $\mathrm{C}_{24} \mathrm{H}_{25} \mathrm{O}_{4}$ Calcd. 377.1753 $(\mathrm{M}+\mathrm{H})$; found: 377.1743 . 


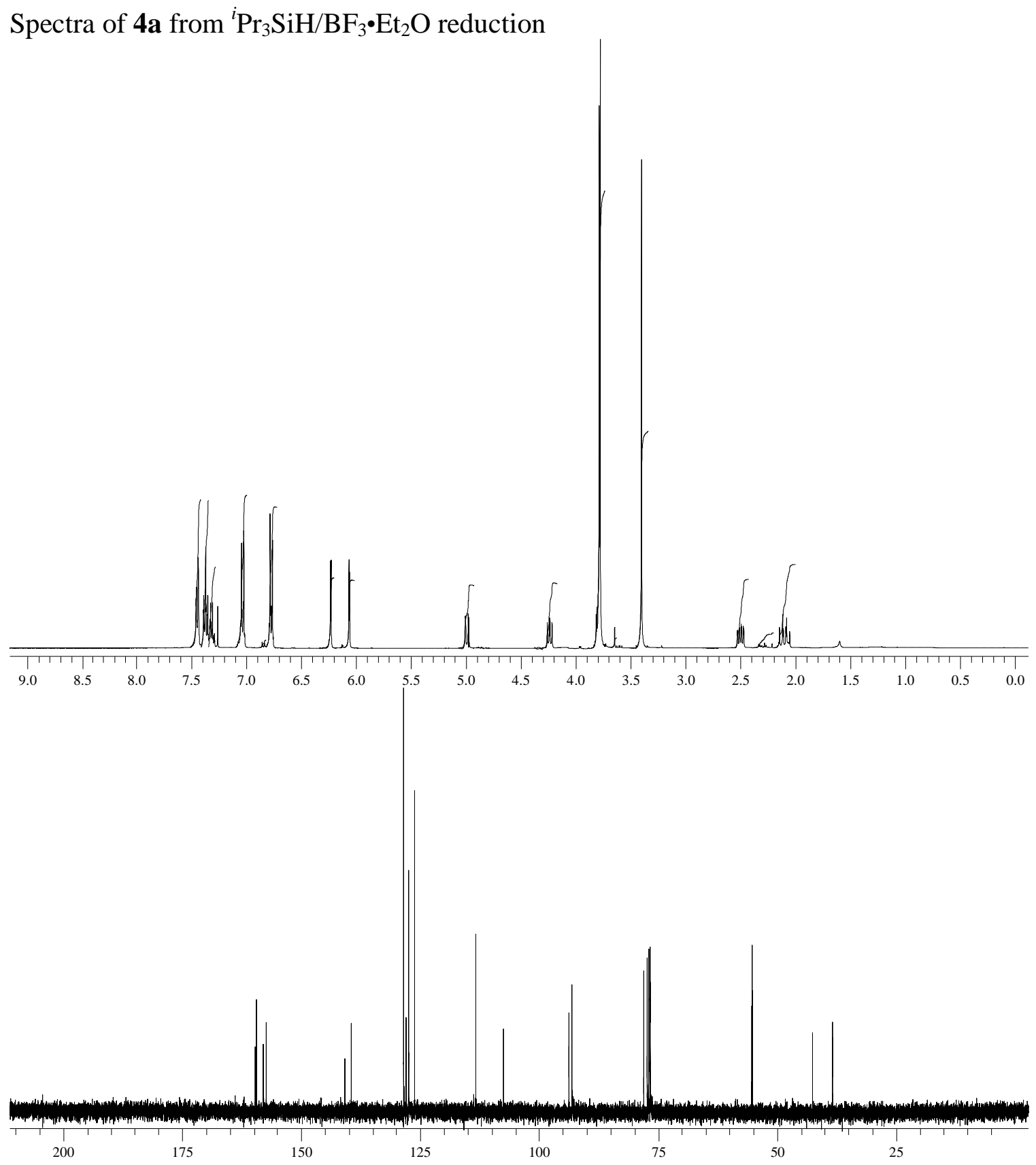


Spectra of $\mathbf{4 a}$ from $\mathrm{PhSiH}_{3} / \mathrm{BF}_{3} \cdot \mathrm{Et}_{2} \mathrm{O}$ reduction

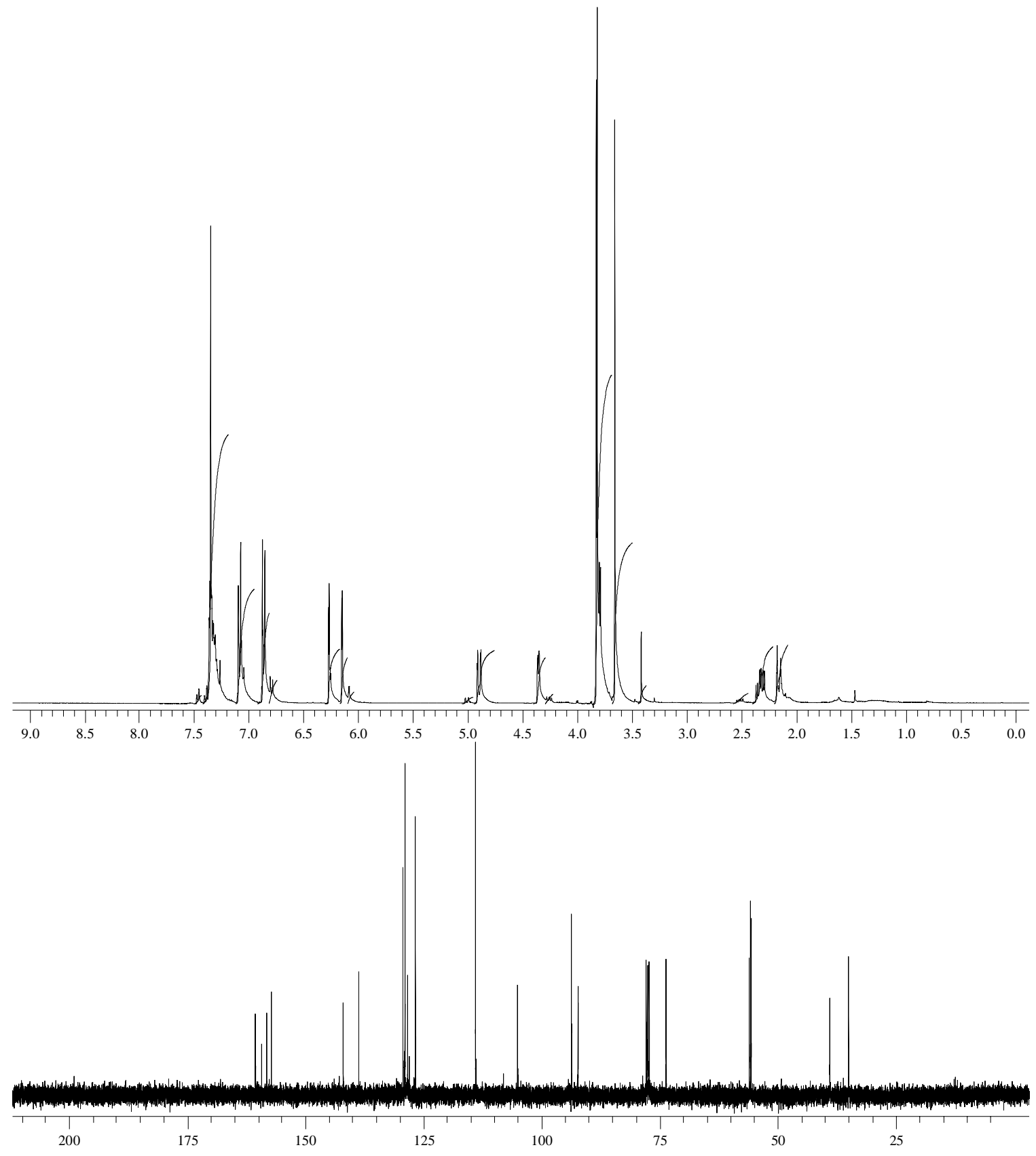

S-28 
Spectra of $\mathbf{4 a}$ from $\mathrm{Et}_{3} \mathrm{SiH} / \mathrm{BF}_{3} \cdot \mathrm{Et}_{2} \mathrm{O}$ reduction

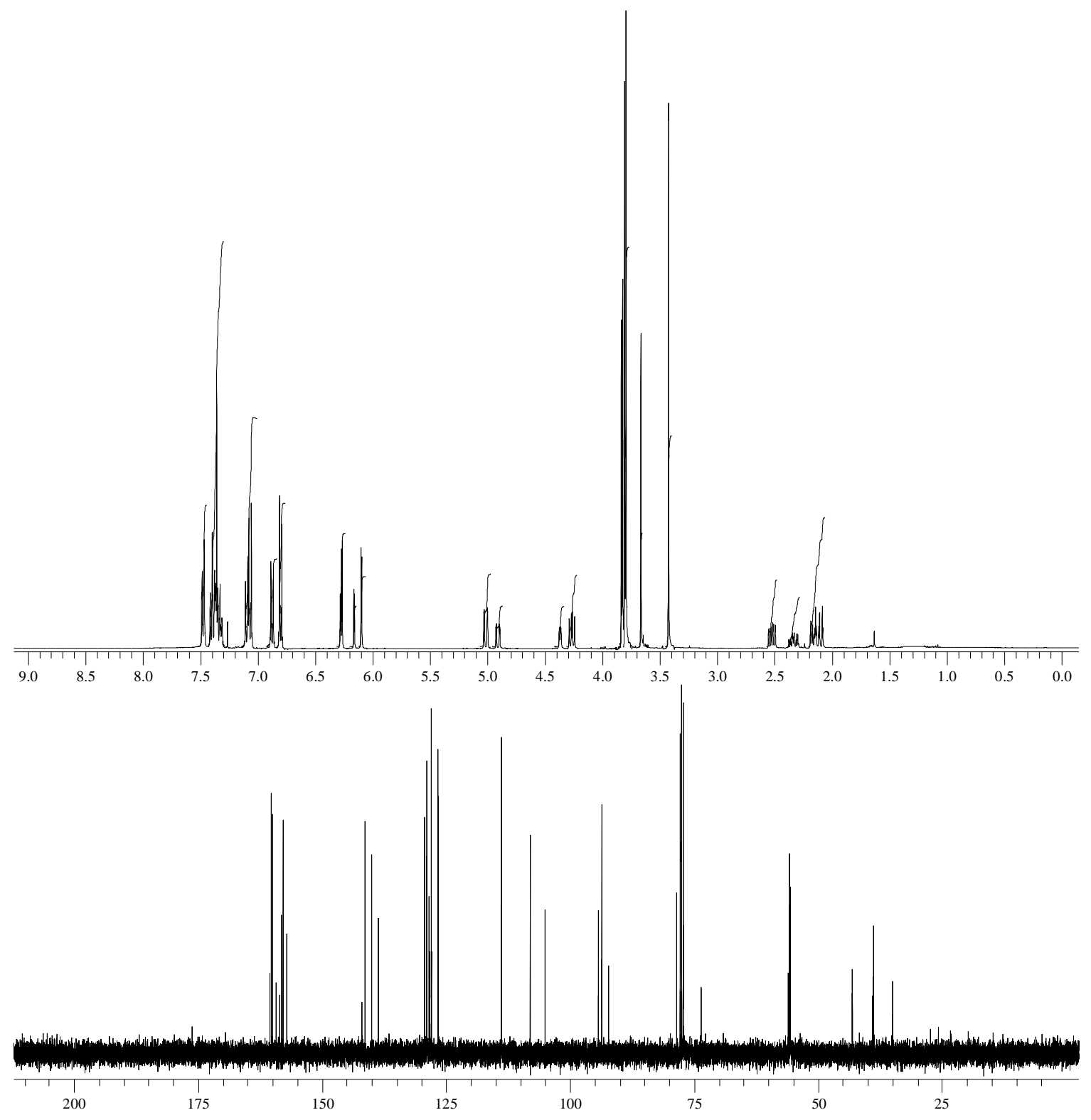



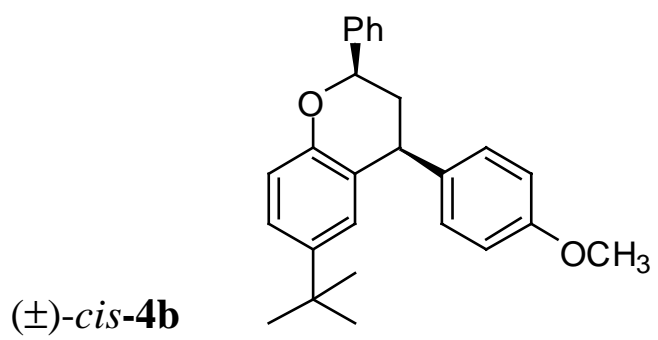

Column chromatography on silica gel $\left(\mathrm{DCM} /\right.$ hexane $\left.=1 / 1, \mathrm{R}_{\mathrm{f}}=0.6\right)$ to afford $4 \mathbf{b}$.

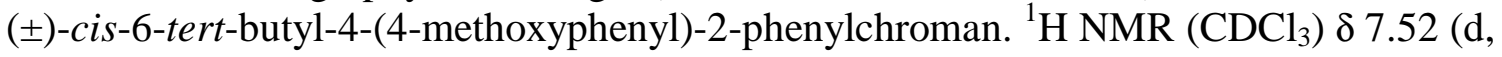
$2 \mathrm{H}, P h \mathrm{CHO}, J=7.0 \mathrm{~Hz}) ; 7.43(\mathrm{t}, 2 \mathrm{H}, P h \mathrm{CHO}, J=7.0 \mathrm{~Hz}) ; 7.36(\mathrm{t}, 1 \mathrm{H}, P h \mathrm{CHO}, J=7.0$ $\mathrm{Hz}) ; 7.23\left(\mathrm{dd}, 1 \mathrm{H}, P h^{\mathrm{t}} \mathrm{Bu}, J=8.6\right.$ and $\left.2.3 \mathrm{~Hz}\right) ; 7.20\left(\mathrm{~d}, 2 \mathrm{H}, P h \mathrm{OCH}_{3}, J=8.7 \mathrm{~Hz}\right) ; 6.96$ $\left(\mathrm{d}, 1 \mathrm{H}, P h^{\mathrm{t}} \mathrm{Bu}, J=8.6 \mathrm{~Hz}\right) ; 6.92\left(\mathrm{~d}, 2 \mathrm{H}, P h \mathrm{OCH}_{3}, J=8.7 \mathrm{~Hz}\right) ; 6.88\left(\mathrm{~d}, 1 \mathrm{H}, P h^{\mathrm{t}} \mathrm{Bu}, J=2.3\right.$ $\mathrm{Hz}) ; 5.24(\mathrm{dd}, 1 \mathrm{H}, \mathrm{PhCHO}, J=11.4$ and $1.8 \mathrm{~Hz}) ; 4.38\left(\mathrm{dd}, 1 \mathrm{H}, \mathrm{CH}_{3} \mathrm{OPhCH}, J=12.1\right.$ and $5.8 \mathrm{~Hz}) ; 3.85$ (s, $\left.3 \mathrm{H}, \mathrm{OCH}_{3}\right) ; 2.42\left(\mathrm{ddd}, 1 \mathrm{H}, \mathrm{CHCH}_{2}, J=13.6,5.8\right.$ and $\left.1.8 \mathrm{~Hz}\right) ; 2.23-2.32$ $\left(\mathrm{m}, 1 \mathrm{H}, \mathrm{CHCH}_{2}\right), 1.23\left(\mathrm{~s}, 9 \mathrm{H},{ }^{\mathrm{t}} \mathrm{Bu}\right)$.

${ }^{13} \mathrm{C}\left\{{ }^{1} \mathrm{H}\right\}$ NMR $\delta 158.2$ (Quat.), 153.2 (Quat.), 143.0 (Quat.), 141.3 (Quat.), 136.5 (Quat.), 129.4 (Arom. CH), 128.5 (Arom. CH), 127.9 (Arom. CH), 126.5 (Arom. CH), 126.0 (Arom. CH), 124.9 (Arom. CH), 124.6 (Arom. CH), 116.2 (Quat.), 113.8 (Arom. CH), $78.0(\mathrm{PhCHO}), 55.2\left(\mathrm{OCH}_{3}\right), 42.8\left(\mathrm{ArCHCH}_{2}\right), 41.1\left(\mathrm{ArCHCH}_{2}\right), 34.0\left(\mathrm{CMe}_{3}\right), 31.4$ $\left(\mathrm{CMe}_{3}\right) . v_{\max }(\mathrm{NaCl}) / \mathrm{cm}^{-1}:$ 3034, 3005, 1612, 1585, 1512, 1497, 1248, 1176, 1130, 1034. HR-MS: $\mathrm{C}_{26} \mathrm{H}_{28} \mathrm{O}_{2}$ Calcd. 372.2089 (M+); found: 372.2076.

\section{$( \pm)-$ trans $-\mathbf{4 b}$}

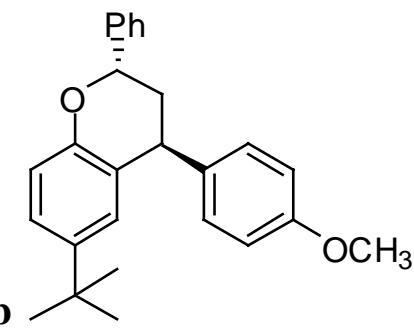

(士)-trans-6-tert-butyl-4-(4-methoxyphenyl)-2-phenylchroman. ${ }^{1} \mathrm{H}$ NMR $\left(\mathrm{CDCl}_{3}\right) \delta$ 7.27$7.37(\mathrm{~m}, 5 \mathrm{H}, P h \mathrm{CHO}) ; 7.21\left(\mathrm{dd}, 1 \mathrm{H}, P h^{\mathrm{t}} \mathrm{Bu}, J=8.6\right.$ and $\left.2.3 \mathrm{~Hz}\right) ; 7.09\left(\mathrm{~d}, 2 \mathrm{H}, P h \mathrm{OCH}_{3}, J\right.$ $=8.7 \mathrm{~Hz}) ; 7.05\left(\mathrm{~d}, 1 \mathrm{H}, P h^{\mathrm{t}} \mathrm{Bu}, J=2.3 \mathrm{~Hz}\right) ; 7.00\left(\mathrm{~d}, 1 \mathrm{H}, P h^{\mathrm{t}} \mathrm{Bu}, J=8.6 \mathrm{~Hz}\right) ; 5.01(\mathrm{dd}, 1 \mathrm{H}$, $\mathrm{PhCHO}, J=10.9$ and $2.3 \mathrm{~Hz}) ; 4.23-4.25\left(\mathrm{~m}, \mathrm{br}, 1 \mathrm{H}, \mathrm{CH}_{3} \mathrm{OPhCH}\right) ; 3.84\left(\mathrm{~s}, 3 \mathrm{H}, \mathrm{OCH}_{3}\right)$; 2.42-2.49 (m, 1H, $\left.\mathrm{CHCH}_{2}\right) ; 1.29$ (s, 9H, $\left.{ }^{\mathrm{t}} \mathrm{Bu}\right) .{ }^{13} \mathrm{C}\left\{{ }^{1} \mathrm{H}\right\} \mathrm{NMR} \delta 158.0$ (Quat.), 153.1 (Quat.), 143.1 (Quat.), 141.6 (Quat.), 138.4 (Quat.), 129.5 (Arom. CH), 128.4 (Arom. $\mathrm{CH}$ ), 127.7 (Arom. $\mathrm{CH}$ ), 127.4 (Arom. $\mathrm{CH}$ ), 125.1 (Arom. $\mathrm{CH}$ ), 122.2 (Arom. $\mathrm{CH}$ ), 116.4 (Quat.), 113.7 (Arom. $\mathrm{CH}), 73.0$ (PhCHO), $55.2\left(\mathrm{OCH}_{3}\right), 39.7\left(\mathrm{ArCHCH}_{2}\right), 38.8$ $(\mathrm{ArCHCH}), 34.0\left(\mathrm{CMe}_{3}\right), 31.5\left(\mathrm{CMe}_{3}\right)$. 
Spectra of $\mathbf{4 b}$ from $\mathrm{Et}_{3} \mathrm{SiH}_{/} \mathrm{BF}_{3} \cdot \mathrm{Et}_{2} \mathrm{O}$ reduction

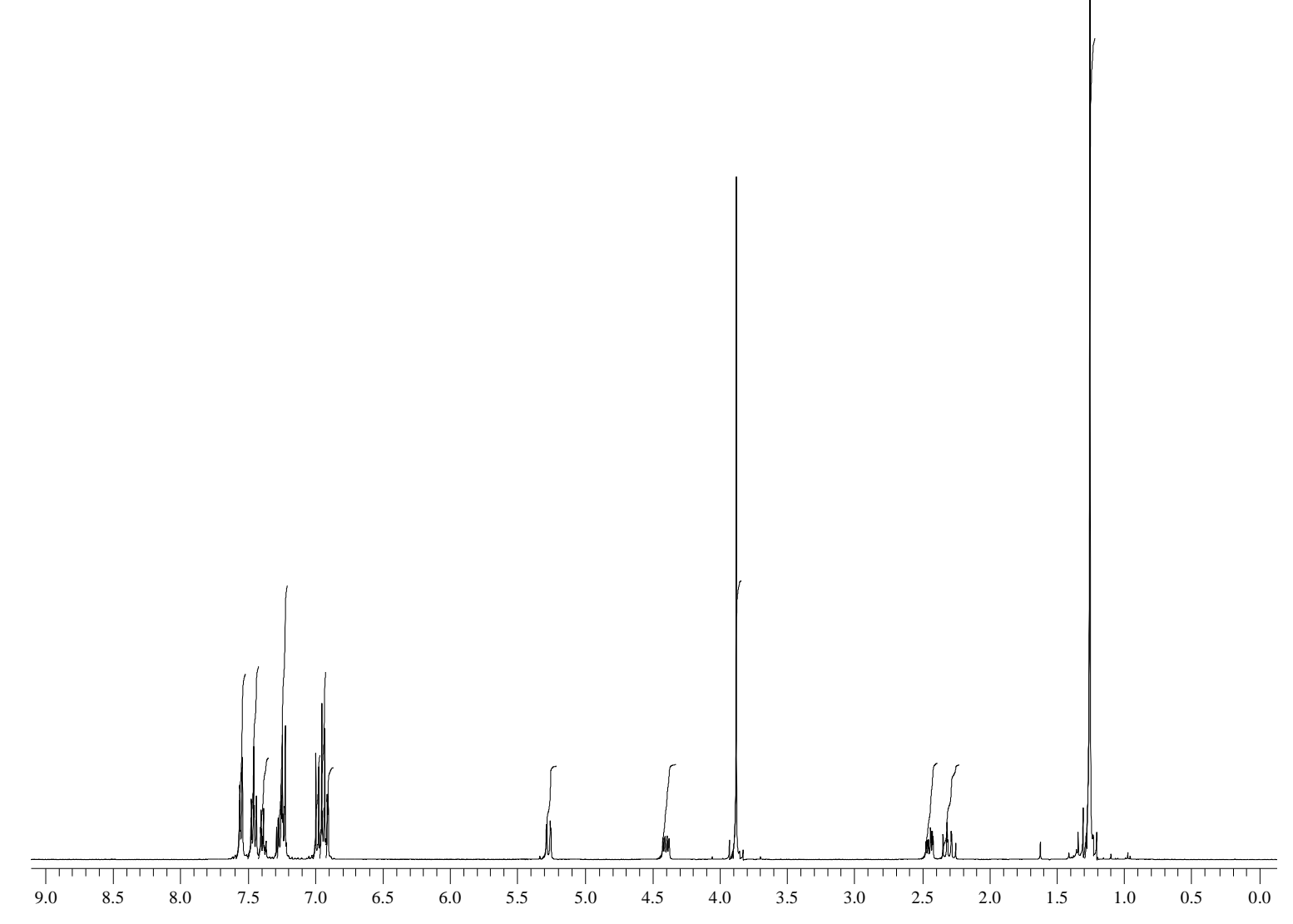

S-31 


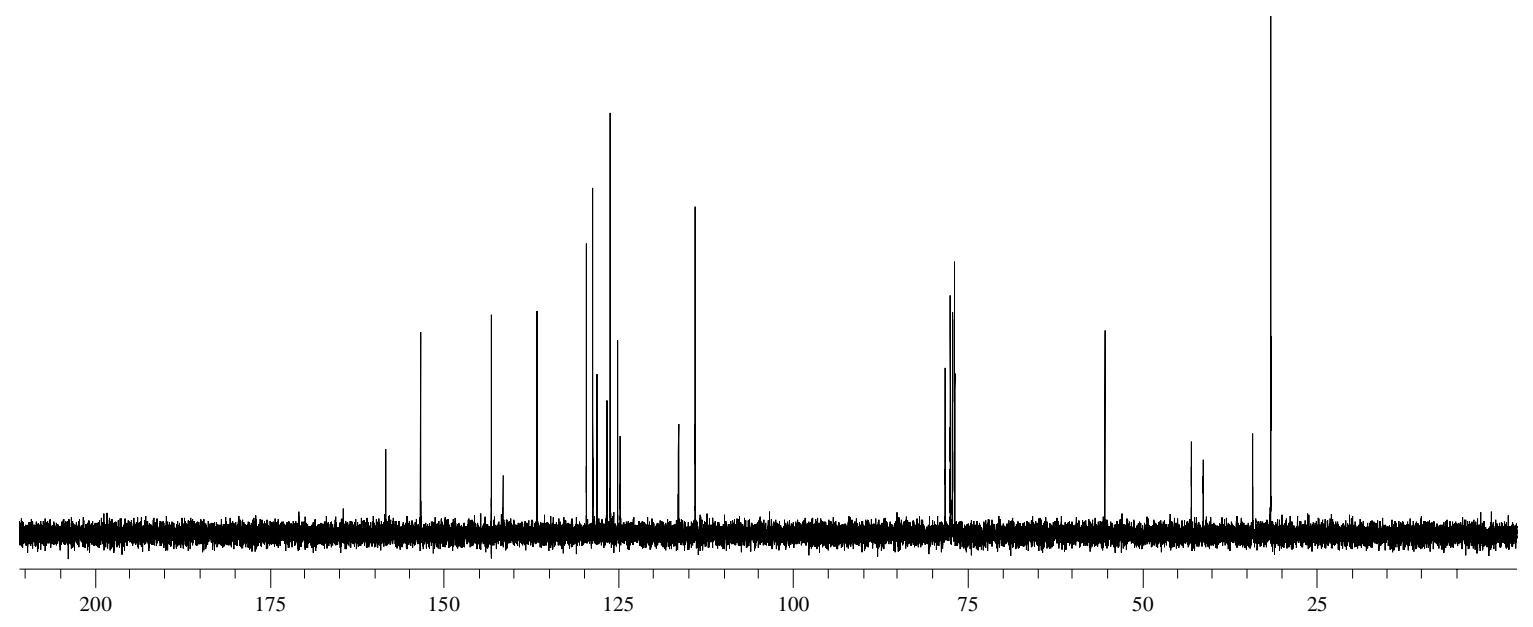

Spectra of $\mathbf{4 b}$ from $\mathrm{PhSiH}_{3} / \mathrm{BF}_{3} \cdot \mathrm{Et}_{2} \mathrm{O}$ reduction 


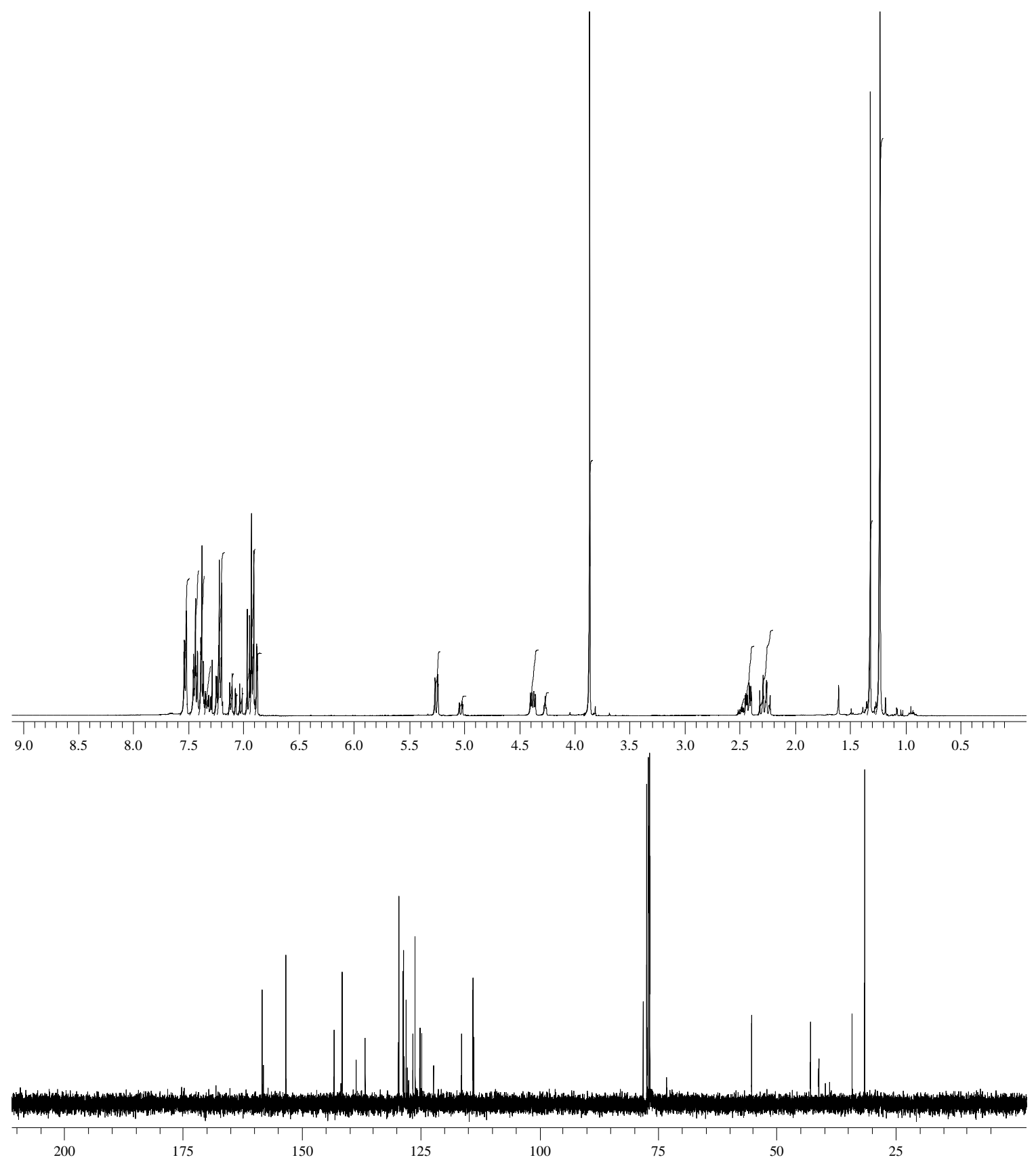

(土)-cis-4c

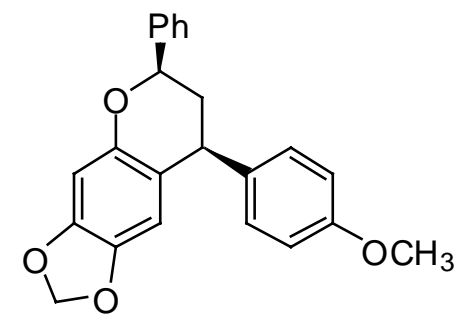

Column chromatography on silica gel $\left(\mathrm{DCM} /\right.$ hexane $\left.=1 / 1, \mathrm{R}_{\mathrm{f}}=0.3\right)$ to afford $4 \mathbf{c}$. 
( \pm )-cis-8-(4-methoxyphenyl)-6-phenyl-7,8-dihydro-6H-[1,3]dioxolo[4,5-g]chromene. ${ }^{1} \mathrm{H}$ NMR $\left(\mathrm{CDCl}_{3}\right) \delta 7.48(\mathrm{~d}, 2 \mathrm{H}, P h \mathrm{CHO}, J=7.1 \mathrm{~Hz}) ; 7.40(\mathrm{t}, 2 \mathrm{H}, P h \mathrm{CHO}, J=7.1 \mathrm{~Hz}) ; 7.33$ (t, $1 \mathrm{H}, P h \mathrm{CHO}, J=7.1 \mathrm{~Hz}) ; 7.14\left(\mathrm{~d}, 2 \mathrm{H}, P h \mathrm{OCH}_{3}, J=8.7 \mathrm{~Hz}\right) ; 6.87\left(\mathrm{~d}, 2 \mathrm{H}, P h \mathrm{OCH}_{3}, J\right.$ $=8.7 \mathrm{~Hz}) ; 6.50\left(\mathrm{~s}, 1 \mathrm{H}, P h \mathrm{OCH}_{2} \mathrm{O}\right) ; 6.24\left(\mathrm{~s}, 1 \mathrm{H}, P h \mathrm{OCH}_{2} \mathrm{O}\right) ; 5.86\left(\mathrm{~d}, 1 \mathrm{H}, \mathrm{OCH}_{2} \mathrm{O}, J=6.7\right.$ $\mathrm{Hz}) ; 5.85\left(\mathrm{~d}, 1 \mathrm{H}, \mathrm{OCH}_{2} \mathrm{O}, J=6.7 \mathrm{~Hz}\right) ; 5.13(\mathrm{dd}, 1 \mathrm{H}, \mathrm{PhCHO}, J=11.4$ and $1.3 \mathrm{~Hz}) ; 4.23$ (dd, $1 \mathrm{H}, \mathrm{CH}_{3} \mathrm{OPhCH}, J=12.1$ and $6.1 \mathrm{~Hz}$ ); $3.81\left(\mathrm{~s}, 3 \mathrm{H}, \mathrm{OCH}_{3}\right) ; 2.37$ (ddd, $1 \mathrm{H}, \mathrm{CHCH}_{2}$, $J=13.6,5.8$ and $1.8 \mathrm{~Hz}) ; 2.15-2.24\left(\mathrm{~m}, 1 \mathrm{H}, \mathrm{CHCH}_{2}\right) .{ }^{13} \mathrm{C}\left\{{ }^{1} \mathrm{H}\right\}$ NMR $\delta 158.3$ (Quat.), 150.2 (Quat.), 146.6 (Quat.), 141.5 (Quat.), 141.1 (Quat.), 136.7 (Quat.), 129.3 (Arom. $\mathrm{CH}$ ), 128.5 (Arom. $\mathrm{CH}$ ), 128.0 (Arom. $\mathrm{CH}$ ), 126.0 (Arom. $\mathrm{CH}$ ), 117.6 (Quat.), 114.0 (Arom. $\mathrm{CH}$ ), 108.3 (Arom. $\mathrm{CH}$ ), $100.8\left(\mathrm{OCH}_{2} \mathrm{O}\right), 98.5$ (Arom. $\mathrm{CH}$ ), $78.2(\mathrm{PhCHO}), 55.2$ $\left.\left(\mathrm{OCH}_{3}\right), 42.6(\mathrm{ArCHCH})_{2}\right), 40.7(\mathrm{ArCHCH}) . v_{\max }(\mathrm{NaCl}) / \mathrm{cm}^{-1}: 3031,3009,1627,1610$, 1512, 1504, 1479, 1247, 1178, 1147, 1039. HR-MS: $\mathrm{C}_{23} \mathrm{H}_{21} \mathrm{O}_{4}$ Calcd. $361.1440(\mathrm{M}+\mathrm{H})$; found: 361.1426 .

$( \pm)-$ trans-4c

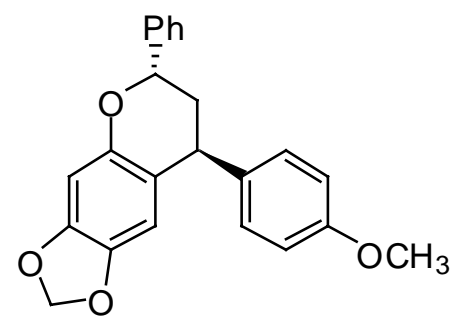

(士)-trans-8-(4-methoxyphenyl)-6-phenyl-7,8-dihydro-6H-[1,3]dioxolo[4,5-g]chromene.

${ }^{1} \mathrm{H}$ NMR $\left(\mathrm{CDCl}_{3}\right) \delta 7.08\left(\mathrm{~d}, 2 \mathrm{H}, P h \mathrm{OCH}_{3}, J=8.8 \mathrm{~Hz}\right) ; 6.56\left(\mathrm{~s}, 1 \mathrm{H}, P h \mathrm{OCH}_{2} \mathrm{O}\right) ; 6.41(\mathrm{~s}$, $\left.1 \mathrm{H}, P h \mathrm{OCH}_{2} \mathrm{O}\right) ; 5.90\left(\mathrm{~d}, 1 \mathrm{H}, \mathrm{OCH}_{2} \mathrm{O}, J=3.1 \mathrm{~Hz}\right) ; 5.89\left(\mathrm{~d}, 1 \mathrm{H}, \mathrm{OCH}_{2} \mathrm{O}, J=3.1 \mathrm{~Hz}\right)$; 4.98 (dd, $1 \mathrm{H}, \mathrm{PhCHO}, J=10.4$ and $2.0 \mathrm{~Hz}$ ); 4.07 (dd, $1 \mathrm{H}, \mathrm{CH}_{3} \mathrm{OPhCH}, J=5.8$ and 3.8 $\mathrm{Hz}) ; 3.81$ (s, 3H, $\left.\mathrm{OCH}_{3}\right)$.

Spectra of $\mathbf{4 c}$ from $\mathrm{Et}_{3} \mathrm{SiH} / \mathrm{BF}_{3} \cdot \mathrm{Et}_{2} \mathrm{O}$ reduction 


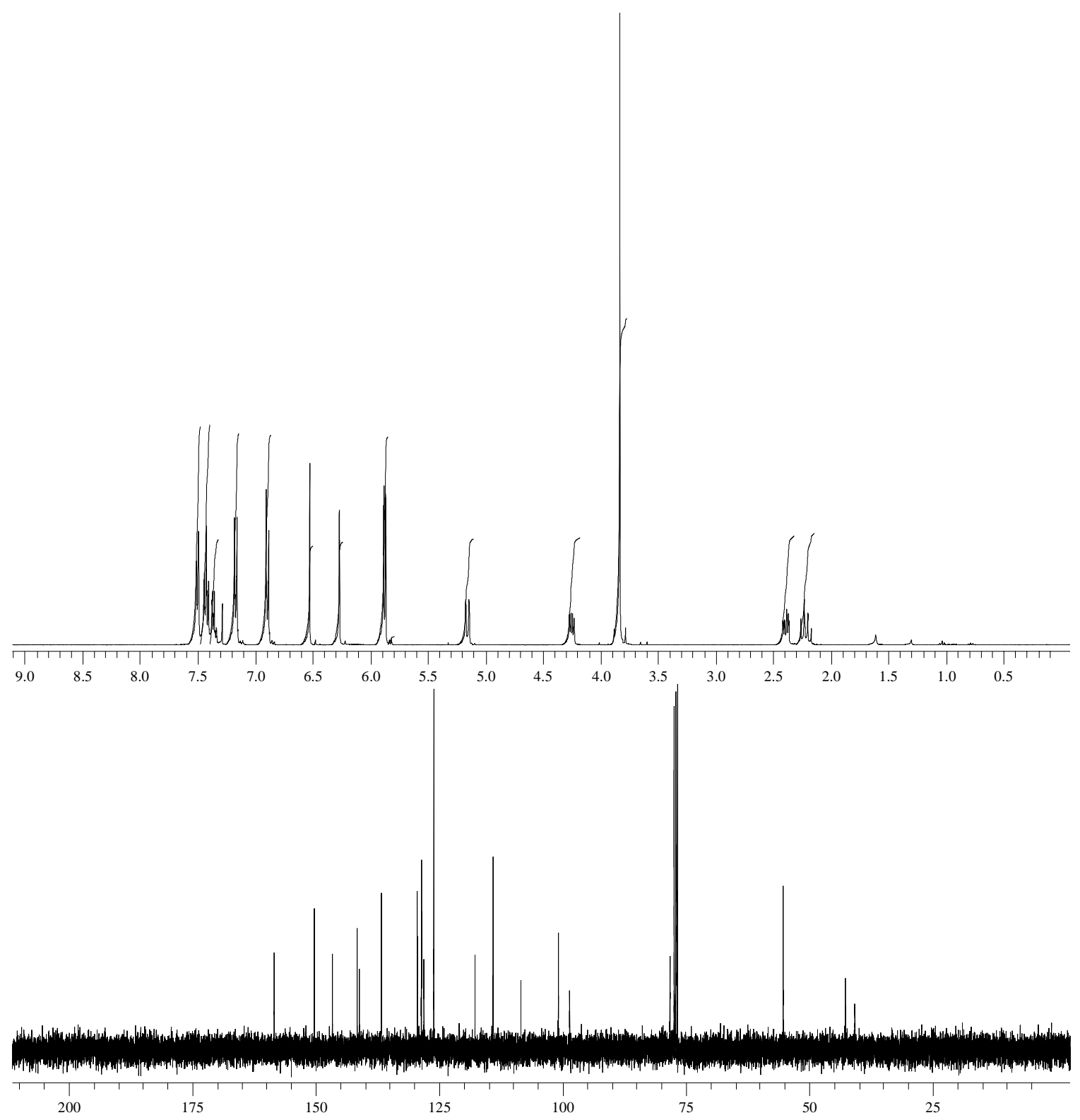

Spectra of $\mathbf{4 c}$ from $\mathrm{PhSiH}_{3} / \mathrm{BF}_{3} \cdot \mathrm{Et}_{2} \mathrm{O}$ reduction 

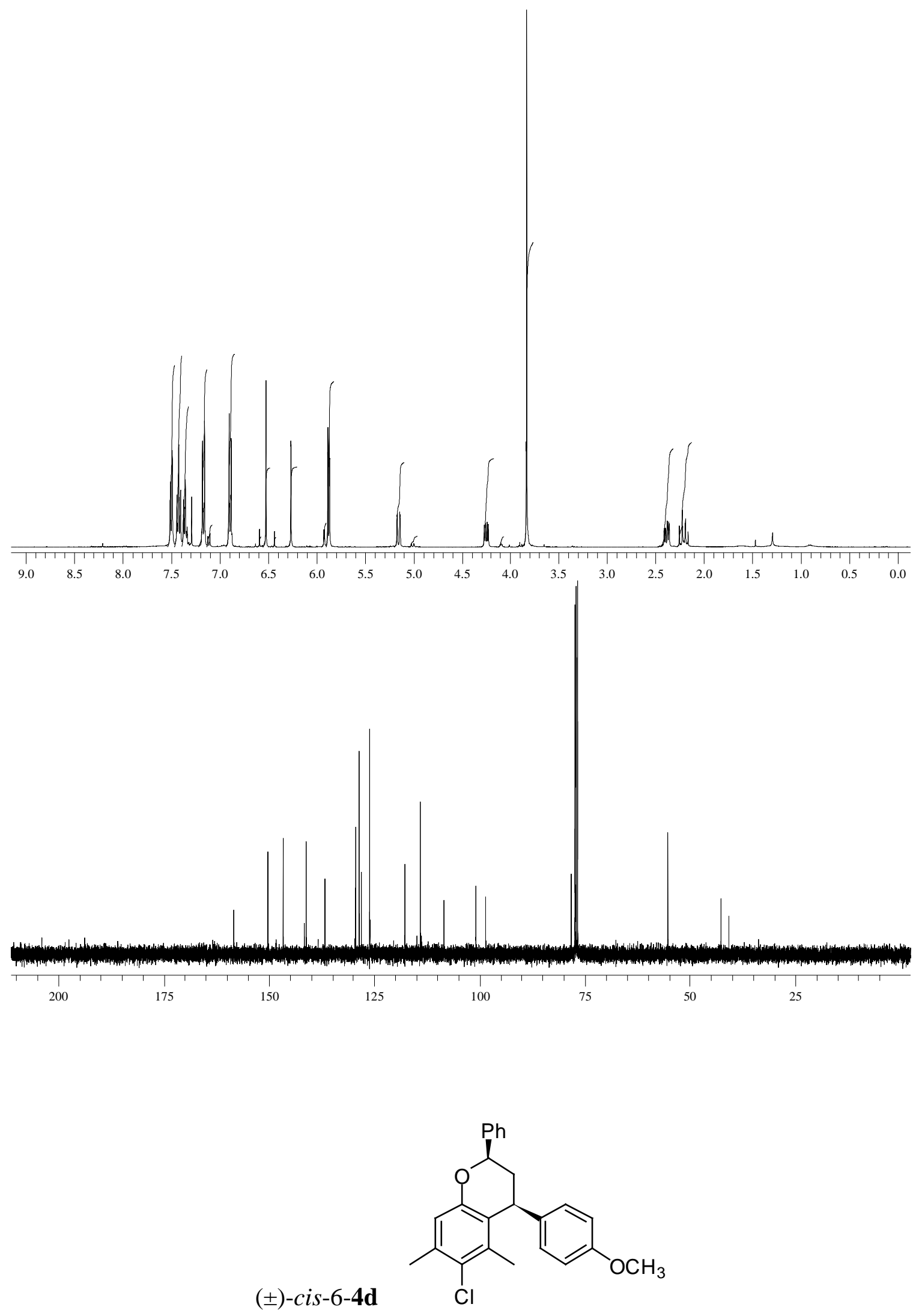

Column chromatography on silica gel $\left(\mathrm{DCM} / \mathrm{hexane}=1 / 1, \mathrm{R}_{\mathrm{f}}=0.6\right)$ to afford $4 \mathbf{d}$. 


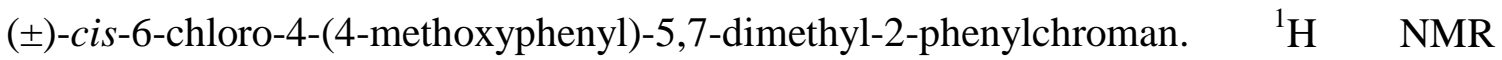
$\left(\mathrm{CDCl}_{3}\right) \delta 7.42(\mathrm{~d}, 2 \mathrm{H}, P h \mathrm{CHO}, J=7.1 \mathrm{~Hz}) ; 7.36(\mathrm{t}, 2 \mathrm{H}, P h \mathrm{CHO}, J=7.1 \mathrm{~Hz}) ; 7.30(\mathrm{t}$, $1 \mathrm{H}, P h \mathrm{CHO}, J=7.1 \mathrm{~Hz}) ; 6.96\left(\mathrm{~d}, 2 \mathrm{H}, P_{h O C H}, J=8.7 \mathrm{~Hz}\right) ; 6.82(\mathrm{~s}, 1 \mathrm{H}, \mathrm{PhCl}), 6.78(\mathrm{~d}$, $\left.2 \mathrm{H}, \mathrm{PhOCH}_{3}, J=8.7 \mathrm{~Hz}\right) ; 4.95(\mathrm{dd}, 1 \mathrm{H}, \mathrm{PhCHO}, J=11.4$ and $1.5 \mathrm{~Hz}) ; 4.34(\mathrm{dd}, 1 \mathrm{H}$, $\mathrm{CH}_{3} \mathrm{OPhCH}, J=10.4$ and $8.1 \mathrm{~Hz}$ ); $3.77\left(\mathrm{~s}, 3 \mathrm{H}, \mathrm{OCH}_{3}\right) ; 2.58\left(\mathrm{ddd}, 1 \mathrm{H}, \mathrm{CHCH}_{2}, J=14.1\right.$, 7.8 and $1.8 \mathrm{~Hz}$ ); 2.36 (s, 3H, $\left.\mathrm{PhCH}_{3}\right) ; 2.08-2.17$ (m, $\left.1 \mathrm{H}, \mathrm{CHCH}_{2}\right) ; 1.90$ (s, 3H, $\left.\mathrm{PhCH}_{3}\right)$. ${ }^{13} \mathrm{C}\left\{{ }^{1} \mathrm{H}\right\}$ NMR $\delta 157.7$ (Quat.), 155.2 (Quat.), 140.6 (Quat.), 138.3 (Quat.), 136.3 (Quat.), 135.5 (Quat.), 128.45 (Arom. CH), 128.40 (Arom. CH), 127.9 (Arom. CH), 127.8 (Quat.), 126.0 (Arom. CH), 123.2 (Quat.), 117.2 (Arom. CH), 114. (Arom. CH)1, 77.4 $(\mathrm{PhCHO}), 55.2\left(\mathrm{OCH}_{3}\right), 43.6\left(\mathrm{ArCHCH}_{2}\right), 41.5\left(\mathrm{ArCHCH}_{2}\right), 20.8\left(\mathrm{ArCH}_{3}\right), 18.6$ $\left(\mathrm{ArCH} \mathrm{H}_{3}\right) . V_{\max }(\mathrm{NaCl}) / \mathrm{cm}^{-1}: 3031,3003,1608,1564,1510,1463,1306,1271,1247,1176$, 1159, 1074, 1033. HR-MS: $\mathrm{C}_{24} \mathrm{H}_{23} \mathrm{ClO}_{2}$ Calcd. 378.1387 (M+); found: 378.1393.

\section{$( \pm)$-trans-4d}<smiles>COc1ccc(C2C[C@H](c3ccccc3)Oc3cc(C)c(Cl)c(C)c32)cc1</smiles>

( \pm )-trans-6-chloro-4-(4-methoxyphenyl)-5,7-dimethyl-2-phenylchroman. $\quad{ }^{1} \mathrm{H} \quad \mathrm{NMR}$ $\left(\mathrm{CDCl}_{3}\right) \delta$ 7.29-7.38 (m, 5H, PhCHO); 7.04 (d, 2H, $\left.P h \mathrm{OCH}_{3}, J=8.7 \mathrm{~Hz}\right) ; 6.88$ (d, 2H, $\left.P_{h O C H}, J=8.7 \mathrm{~Hz}\right) ; 6.86(\mathrm{~s}, 1 \mathrm{H}, \mathrm{PhCl}), 4.88(\mathrm{dd}, 1 \mathrm{H}, \mathrm{PhCHO}, J=11.9$ and $2.0 \mathrm{~Hz})$; 4.27 (d, br, $\left.1 \mathrm{H}, \mathrm{CH}_{3} \mathrm{OPhCH}, J=4.0 \mathrm{~Hz}\right) ; 3.82\left(\mathrm{~s}, 3 \mathrm{H}, \mathrm{OCH}_{3}\right) ; 2.41$ (s, 3H, $\left.\mathrm{PhCH}_{3}\right) ; 2.31-$ $2.39\left(\mathrm{~m}, 1 \mathrm{H}, \mathrm{CHCH}_{2}\right) ; 2.18-2.23\left(\mathrm{~m}, 1 \mathrm{H}, \mathrm{CHCH}_{2}\right) ; 2.11\left(\mathrm{~s}, 3 \mathrm{H}, \mathrm{PhCH}_{3}\right) .{ }^{13} \mathrm{C}\left\{{ }^{1} \mathrm{H}\right\} \mathrm{NMR} \delta$ 158.1 (Quat.), 153.5 (Quat.), 141.3 (Quat.), 136.9 (Quat.), 135.9 (Quat.), 135.5 (Quat.), 129.2 (Arom. CH), 128.4 (Arom. CH), 127.8 (Arom. CH), 126.8 (Quat.), 126.1 (Arom. $\mathrm{CH}$ ), 120.4 (Quat.), 116.8 (Arom. $\mathrm{CH}$ ), 113.9 (Arom. $\mathrm{CH}$ ), 72.2 (PhCHO), $55.2\left(\mathrm{OCH}_{3}\right)$, $39.0\left(\mathrm{ArCHCH}_{2}\right), 38.8\left(\mathrm{ArCHCH}_{2}\right), 20.9\left(\mathrm{ArCH}_{3}\right), 16.4\left(\mathrm{ArCH}_{3}\right) . v \max (\mathrm{NaCl}) / \mathrm{cm}^{-1}: 3032$, 3005, 1609, 1581, 1566, 1510, 1456, 1303, 1247, 1174, 1166, 1093, 1036. HR-MS: $\mathrm{C}_{24} \mathrm{H}_{23} \mathrm{ClO}_{2}$ Calcd. $378.1387(\mathrm{M}+)$; found: 378.1391.

Spectra of $\mathbf{4 d}$ from ${ }^{i} \mathrm{Pr}_{3} \mathrm{SiH} / \mathrm{BF}_{3} \cdot \mathrm{Et}_{2} \mathrm{O}$ reduction 


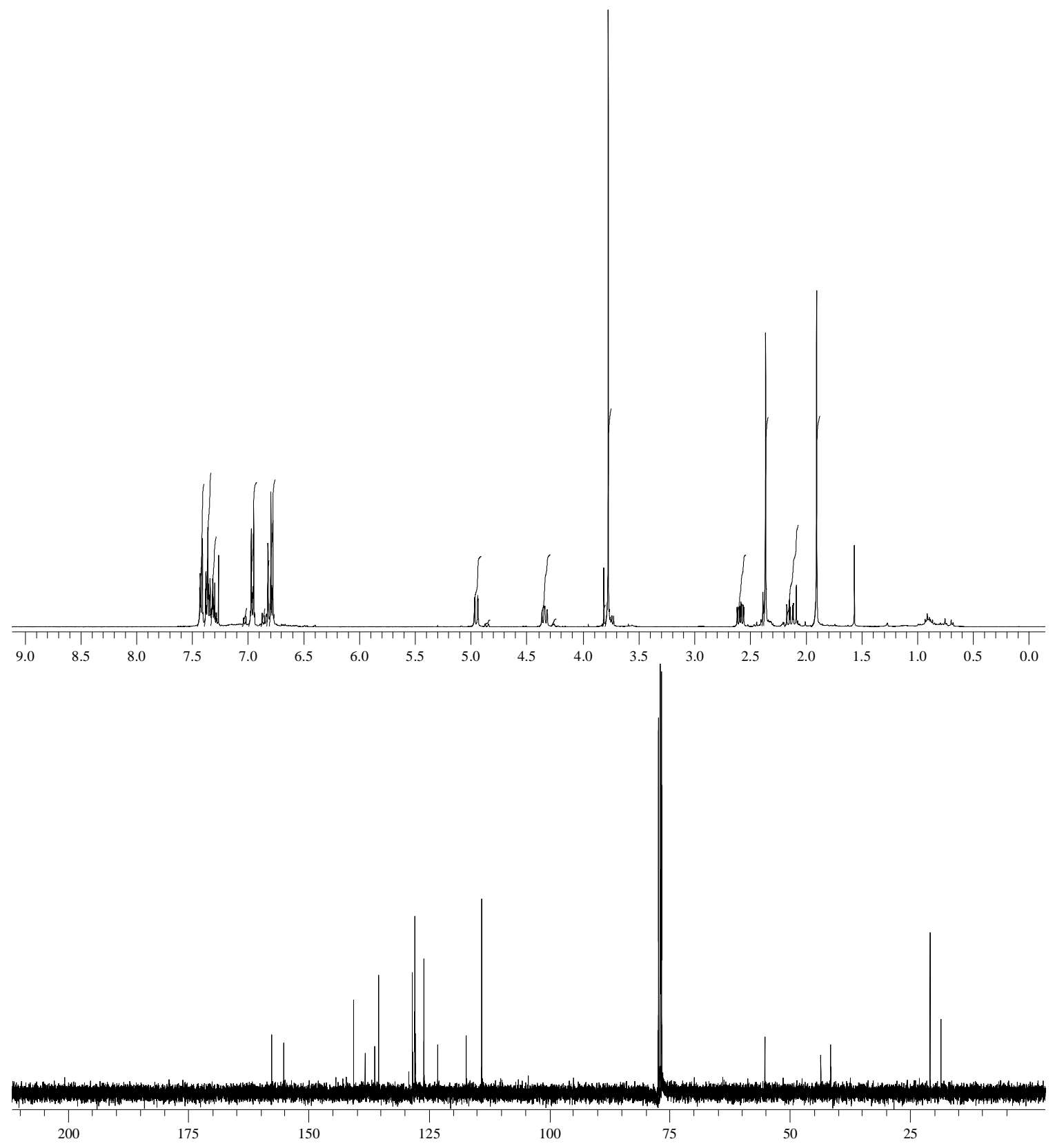

Spectra of $\mathbf{4 d}$ from $\mathrm{PhSiH}_{3} / \mathrm{BF}_{3} \cdot \mathrm{Et}_{2} \mathrm{O}$ reduction

S-38 


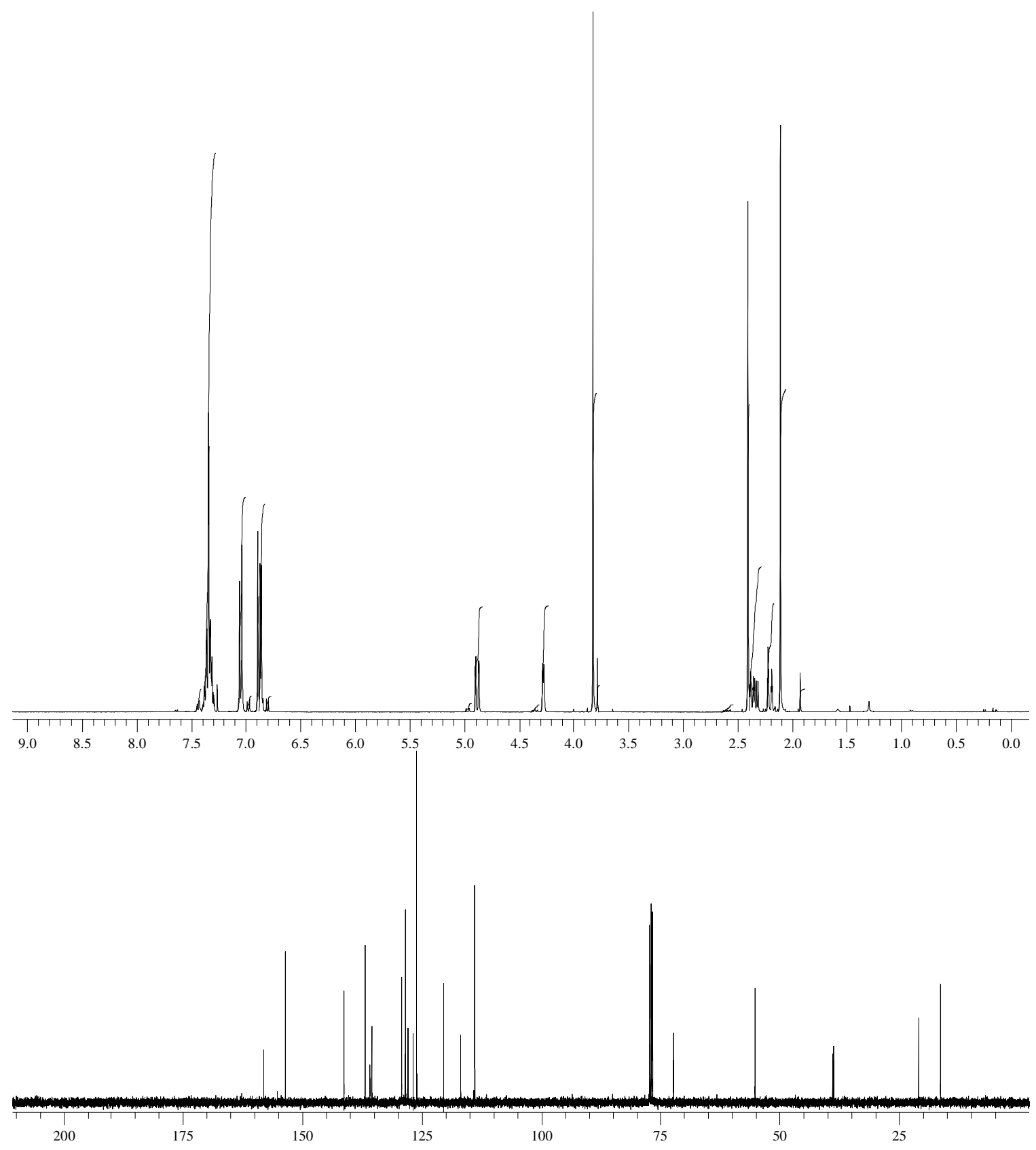

$( \pm)-c i s-\mathbf{4 e}$<smiles>COc1ccc(C2C[C@H](c3ccccc3)Oc3c(C(C)C)ccc(C)c32)cc1</smiles>

S-39 
Column chromatography on silica gel (DCM/hexane $\left.=1 / 1, \mathrm{R}_{\mathrm{f}}=0.6\right)$ to afford $4 \mathbf{e}$.

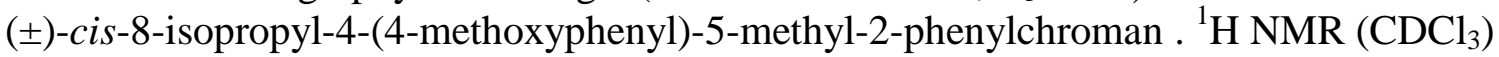
$\delta 7.52(\mathrm{~d}, 2 \mathrm{H}, P h \mathrm{CHO}, J=7.3 \mathrm{~Hz}) ; 7.42(\mathrm{t}, 2 \mathrm{H}, P h \mathrm{CHO}, J=7.3 \mathrm{~Hz}) ; 7.34(\mathrm{t}, 1 \mathrm{H}$, $P h \mathrm{CHO}, J=7.3 \mathrm{~Hz}) ; 7.11\left(\mathrm{~d}, 1 \mathrm{H}, P h \mathrm{CH}_{3}, J=7.7 \mathrm{~Hz}\right) ; 7.04\left(\mathrm{~d}, 2 \mathrm{H}, P h \mathrm{OCH}_{3}, J=8.7\right.$ $\mathrm{Hz}) ; 6.82\left(\mathrm{~d}, 2 \mathrm{H}, P \mathrm{OCH}_{3}, J=8.7 \mathrm{~Hz}\right) ; 6.76\left(\mathrm{~d}, 1 \mathrm{H}, P h \mathrm{CH}_{3}, J=7.7 \mathrm{~Hz}\right) ; 5.03(\mathrm{dd}, 1 \mathrm{H}$, $\mathrm{PhCHO}, J=11.4$ and $1.5 \mathrm{~Hz}) ; 4.40\left(\mathrm{dd}, 1 \mathrm{H}, \mathrm{CH}_{3} \mathrm{OPhCH}, J=10.6\right.$ and $\left.8.3 \mathrm{~Hz}\right) ; 3.80$ (s, $\left.3 \mathrm{H}, \mathrm{OCH}_{3}\right) ; 3.40-3.49\left(\mathrm{~m}, 1 \mathrm{H}, \mathrm{CH}\left(\mathrm{CH}_{3}\right)_{2}\right), 2.69\left(\mathrm{ddd}, 1 \mathrm{H}, \mathrm{CHCH}_{2}, J=13.9,8.1\right.$ and 1.8 $\mathrm{Hz}) ; 2.12-2.21\left(\mathrm{~m}, 1 \mathrm{H}, \mathrm{CHCH}_{2}\right) ; 1.85\left(\mathrm{~s}, 3 \mathrm{H}, \mathrm{PhCH}_{3}\right) ; 1.36\left(\mathrm{~d}, 3 \mathrm{H}, \mathrm{CH}\left(\mathrm{CH}_{3}\right)_{2}, J=7.1\right.$ $\mathrm{Hz}$ ), 1.27 (d, 3H, $\left.\mathrm{CH}\left(\mathrm{CH}_{3}\right)_{2}, J=7.1 \mathrm{~Hz}\right) .{ }^{13} \mathrm{C}\left\{{ }^{1} \mathrm{H}\right\} \mathrm{NMR} \delta 157.6$ (Quat.), 154.3 (Quat.), 141.5 (Quat.), 138.8 (Quat.), 134.6 (Quat.), 128.32 (Arom. CH), 128.1 (Arom. CH), 127.5 (Arom. $\mathrm{CH}$ ), 125.7 (Arom. $\mathrm{CH}$ ), 124.0 (Arom. $\mathrm{CH}$ ), 123.7 (Quat.), 123.1 (Arom. $\mathrm{CH}), 113.9$ (Arom. $\mathrm{CH}), 76.9$ (PhCHO), $55.1 \quad\left(\mathrm{OCH}_{3}\right), 44.0\left(\mathrm{ArCHCH}_{2}\right), 41.3$ $\left.(\mathrm{ArCHCH})_{2}\right), 26.7\left(\mathrm{CH}\left(\mathrm{CH}_{3}\right)_{2}\right), 23.0\left(\mathrm{CH}\left(\mathrm{CH}_{3}\right)_{2}\right), 22.6\left(\mathrm{CH}\left(\mathrm{CH}_{3}\right)_{2}\right), 20.8\left(\mathrm{PhCH}_{3}\right) . v_{\max }$ $(\mathrm{NaCl}) / \mathrm{cm}^{-1}:$ 3032, 3003, 1609, 1583, 1508, 1481, 1454, 1444, 1418, 1332, 1244, 1176 , 1062, 1036. HR-MS: $\mathrm{C}_{26} \mathrm{H}_{29} \mathrm{O}_{2}$ Calcd. $373.2168(\mathrm{M}+\mathrm{H})$; found: 373.2163.

$( \pm)-$ trans-4e

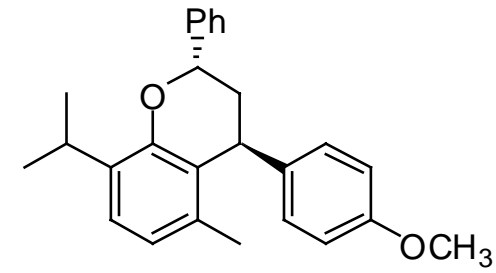

(士)-trans-8-Isopropyl-4-(4-methoxyphenyl)-5-methyl-2-phenylchroman). $\quad{ }^{1} \mathrm{H} \quad \mathrm{NMR}$ $\left(\mathrm{CDCl}_{3}\right) \delta$ 7.38-7.42 (m, 4H, PhCHO); 7.30-7.36 (m, 1H, PhCHO); 7.17 (d, $1 \mathrm{H}, P h \mathrm{CH}_{3}$, $J=7.6 \mathrm{~Hz}) ; 7.06\left(\mathrm{~d}, 2 \mathrm{H}, P h \mathrm{OCH}_{3}, J=8.8 \mathrm{~Hz}\right) ; 6.90$ (d, $\left.2 \mathrm{H}, P h \mathrm{OCH}_{3}, J=8.8 \mathrm{~Hz}\right) ; 6.82$ $\left(\mathrm{d}, 1 \mathrm{H}, \mathrm{PhCH}_{3}, J=7.6 \mathrm{~Hz}\right) ; 4.97(\mathrm{dd}, 1 \mathrm{H}, \mathrm{PhCHO}, J=11.9$ and $2.0 \mathrm{~Hz}) ; 4.28(\mathrm{~d}, \mathrm{br}, 1 \mathrm{H}$, $\left.\mathrm{CH}_{3} \mathrm{OPhCH}, J=3.8 \mathrm{~Hz}\right) ; 3.84\left(\mathrm{~s}, 3 \mathrm{H}, \mathrm{OCH}_{3}\right) ; 3.42-3.51\left(\mathrm{~m}, 1 \mathrm{H}, \mathrm{CH}\left(\mathrm{CH}_{3}\right)_{2}\right), 2.31-2.39$ $\left(\mathrm{m}, 1 \mathrm{H}, \mathrm{CHCH}_{2}\right) ; 2.21-2.25\left(\mathrm{~m}, 1 \mathrm{H}, \mathrm{CHCH}_{2}\right) ; 2.03\left(\mathrm{~s}, 3 \mathrm{H}, \mathrm{PhCH}_{3}\right) ; 1.34(\mathrm{~d}, 3 \mathrm{H}$, $\left.\mathrm{CH}\left(\mathrm{CH}_{3}\right)_{2}, J=6.8 \mathrm{~Hz}\right), 1.32\left(\mathrm{~d}, 3 \mathrm{H}, \mathrm{CH}\left(\mathrm{CH}_{3}\right)_{2}, J=6.8 \mathrm{~Hz}\right) .{ }^{13} \mathrm{C}\left\{{ }^{1} \mathrm{H}\right\} \mathrm{NMR} \delta 157.9$ (Quat.), 152.4 (Quat.), 142.2 (Quat.), 137.5 (Quat.), 135.1 (Quat.), 134.1 (Quat.), 129.3 (Arom. CH), 128.3 (Arom. CH), 127.4 (Arom. CH), 125.8 (Arom. CH), 124.1 (Arom. $\mathrm{CH}$ ), 121.8 (Arom. CH), 120.9 (Quat.), 113.8 (Arom. $\mathrm{CH}$ ), 71.9 (PhCHO), $55.2\left(\mathrm{OCH}_{3}\right)$, $39.2\left(\mathrm{ArCHCH}_{2}\right), \quad 38.4\left(\mathrm{ArCHCH}_{2}\right), \quad 26.5\left(\mathrm{CH}\left(\mathrm{CH}_{3}\right)_{2}\right), \quad 23.1 \quad\left(\mathrm{CH}\left(\mathrm{CH}_{3}\right)_{2}\right), \quad 22.6$ $\left(\mathrm{CH}\left(\mathrm{CH}_{3}\right)_{2}\right), 18.9\left(\mathrm{ArCH}_{3}\right) . v_{\max }(\mathrm{NaCl}) / \mathrm{cm}^{-1}: 3032,3005,1609,1582,1508,1483,1454$, 1442, 1247, 1178, 1062, 1034. HR-MS: $\mathrm{C}_{26} \mathrm{H}_{29} \mathrm{O}_{2}$ Calcd. $373.2168(\mathrm{M}+\mathrm{H})$; found: 373.2171 .

Spectra of $4 \mathbf{e}$ from ${ }^{i} \mathrm{Pr}_{3} \mathrm{SiH} / \mathrm{BF}_{3} \cdot \mathrm{Et}_{2} \mathrm{O}$ reduction 


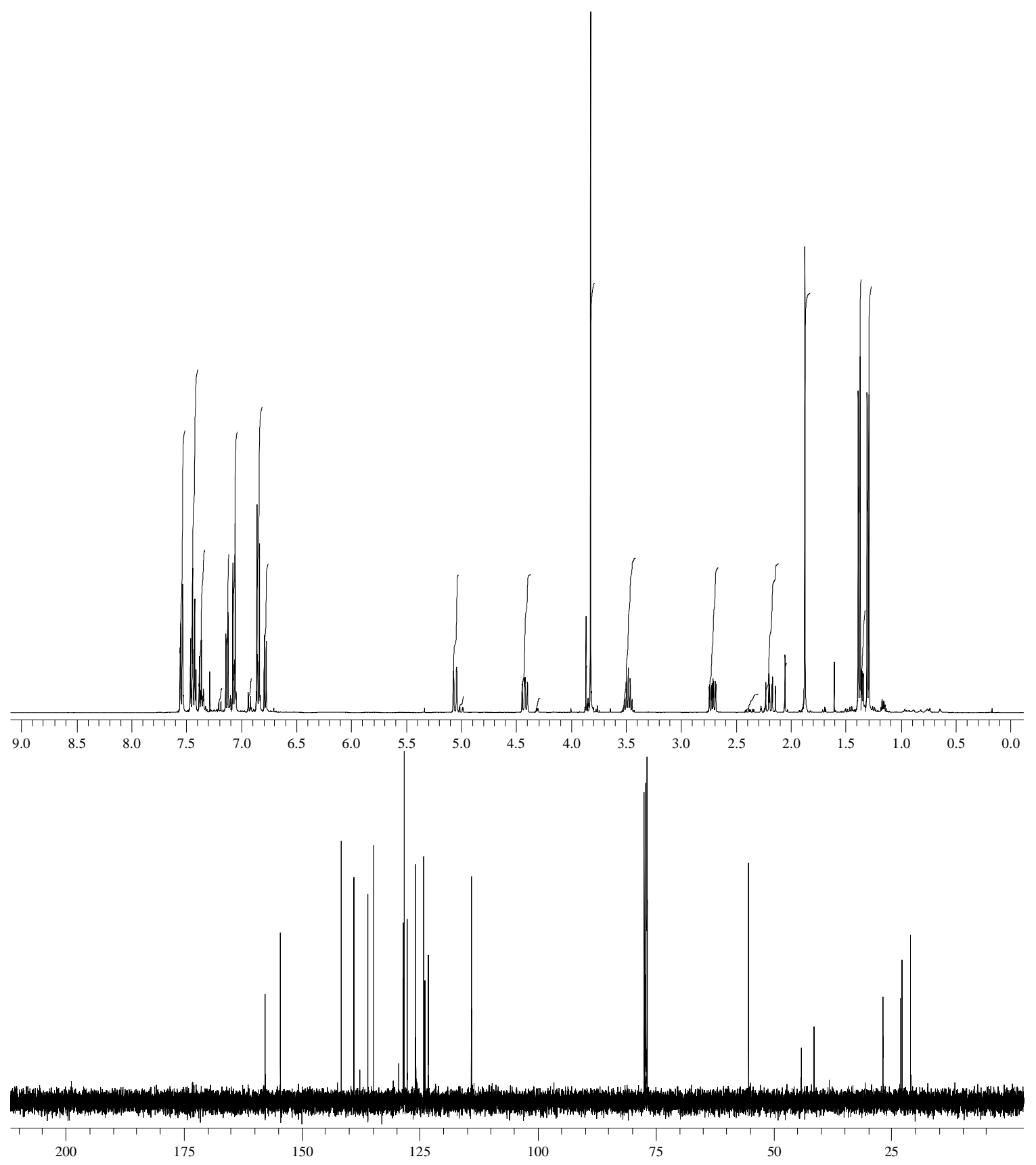

Spectra of $4 \mathbf{e}$ from $\mathrm{Et}_{3} \mathrm{SiH} / \mathrm{BF}_{3} \cdot \mathrm{Et}_{2} \mathrm{O}$ reduction 


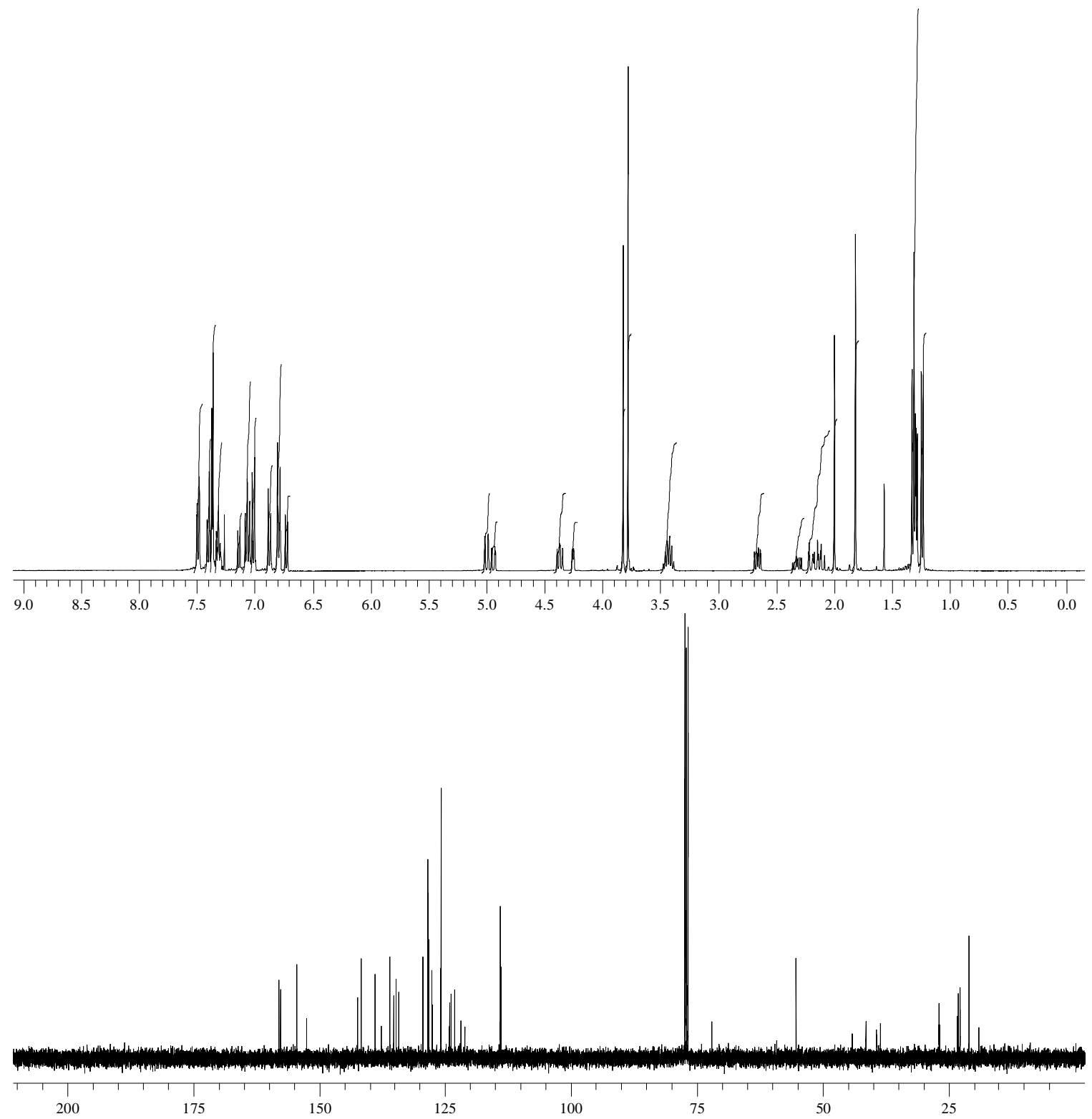

Spectra of $\mathbf{4 e}$ from $\mathrm{PhSiH}_{3} / \mathrm{BF}_{3} \cdot \mathrm{Et}_{2} \mathrm{O}$ reduction 


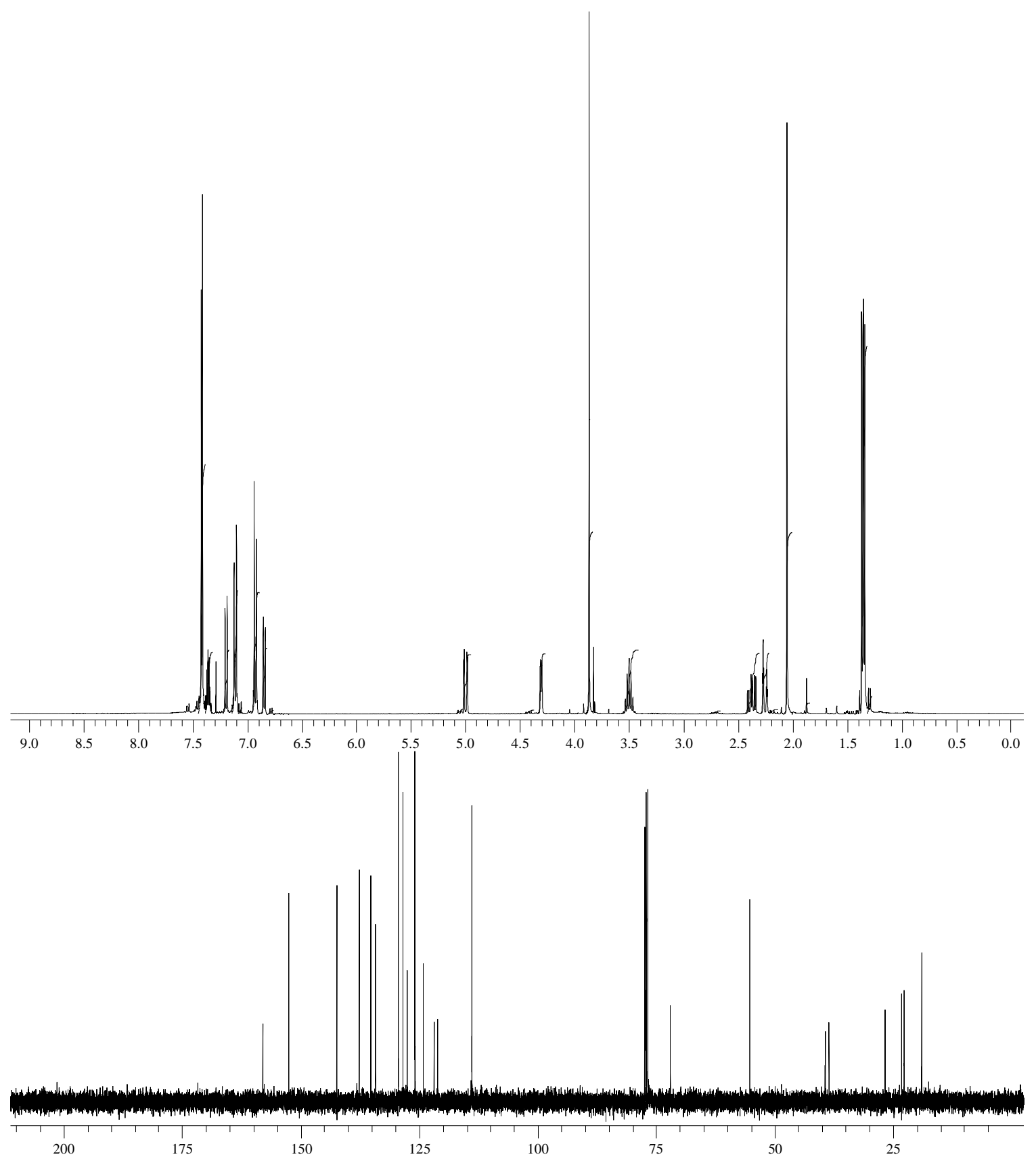

(士)-cis-4f<smiles>COc1ccc(C2CC(c3ccccc3)Oc3ccc(OC)cc32)cc1</smiles> 
Column chromatography on silica gel $\left(\mathrm{DCM} /\right.$ hexane $\left.=1 / 1, \mathrm{R}_{\mathrm{f}}=0.3\right)$ to afford $4 \mathbf{f}$.

(士)-cis-6-methoxy-4-(4-methoxyphenyl)-2-phenylchroman . ${ }^{1} \mathrm{H} \mathrm{NMR}\left(\mathrm{CDCl}_{3}\right) \delta 7.50(\mathrm{~d}$, $2 \mathrm{H}, P h \mathrm{CHO}, J=7.1 \mathrm{~Hz}) ; 7.41(\mathrm{t}, 2 \mathrm{H}, P h \mathrm{CHO}, J=7.1 \mathrm{~Hz}) ; 7.34(\mathrm{t}, 1 \mathrm{H}, P h \mathrm{CHO}, J=7.1$ $\mathrm{Hz}) ; 7.17\left(\mathrm{~d}, 2 \mathrm{H}, \mathrm{PhOCH}_{3}, J=8.7 \mathrm{~Hz}\right) ; 6.92\left(\mathrm{~d}, 1 \mathrm{H}, \mathrm{PhOPhOCH}_{3}, J=8.6 \mathrm{~Hz}\right) ; 6.88(\mathrm{~d}$, $\left.2 \mathrm{H}, P h \mathrm{OCH}_{3}, J=8.7 \mathrm{~Hz}\right) ; 6.75\left(\mathrm{dd}, 1 \mathrm{H}, \mathrm{PhOPhOCH}_{3}, J=8.6\right.$ and $\left.3.0 \mathrm{~Hz}\right) ; 6.36(\mathrm{~d}, 1 \mathrm{H}$, $\left.\mathrm{PhOPhOCH}_{3}, J=3.0 \mathrm{~Hz}\right) ; 5.17(\mathrm{dd}, 1 \mathrm{H}, \mathrm{PhCHO}, J=11.4$ and $1.8 \mathrm{~Hz}) ; 4.31(\mathrm{dd}, 1 \mathrm{H}$, $\mathrm{CH}_{3} \mathrm{OPhCH}, J=12.1$ and $\left.5.8 \mathrm{~Hz}\right) ; 3.82\left(\mathrm{~s}, 3 \mathrm{H}, \mathrm{OCH}_{3}\right) ; 3.65\left(\mathrm{~s}, 3 \mathrm{H}, \mathrm{OCH}_{3}\right) ; 2.36-2.42$ $\left(\mathrm{m}, 1 \mathrm{H}, \mathrm{CHCH}_{2}\right) ; 2.20-2.30\left(\mathrm{~m}, 1 \mathrm{H}, \mathrm{CHCH}_{2}\right) .{ }^{13} \mathrm{C}\left\{{ }^{1} \mathrm{H}\right\}$ NMR $\delta 158.3$ (Quat.), 153.3 (Quat.), 149.5 (Quat.), 141.3 (Quat.), 136.3 (Quat.), 129.4 (Arom. CH), 128.5 (Arom. $\mathrm{CH}$ ), 127.9 (Arom. CH), 126.6 (Quat.), 126.0 (Arom. CH), 117.4 (Arom. CH), 114.5 (Arom. CH), 114.0 (Arom. $\mathrm{CH}$ ), 113.5 (Arom. $\mathrm{CH}$ ), $78.0(\mathrm{PhCHO}), 55.6\left(\mathrm{OCH}_{3}\right), 55.2$ $\left(\mathrm{OCH}_{3}\right), 42.8(\mathrm{ArCHCH}), 40.7(\mathrm{ArCHCH}) . v_{\max }(\mathrm{NaCl}) / \mathrm{cm}^{-1}: 3034,3003,1610,1512$, 1490, 1249, 1209, 1176, 1036. HR-MS: $\mathrm{C}_{23} \mathrm{H}_{23} \mathrm{O}_{3}$ Calcd. $347.1647(\mathrm{M}+\mathrm{H})$; found: 347.1646.

\section{$( \pm)-$ trans-4f}

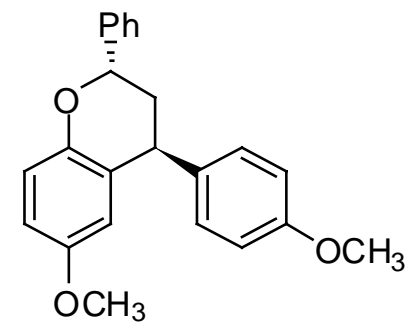

(士)-trans-6-Methoxy-4-(4-methoxyphenyl)-2-phenylchroman.${ }^{1} \mathrm{H} \mathrm{NMR}\left(\mathrm{CDCl}_{3}\right) \delta 7.09$ $\left(\mathrm{d}, 2 \mathrm{H}, P h \mathrm{OCH}_{3}, J=8.6 \mathrm{~Hz}\right) ; 6.98\left(\mathrm{~d}, 1 \mathrm{H}, \mathrm{PhOPhOCH}_{3}, J=8.8 \mathrm{~Hz}\right) ; 6.83(\mathrm{dd}, 1 \mathrm{H}$, $\mathrm{PhOPhOCH}_{3}, J=8.8$ and $\left.3.0 \mathrm{~Hz}\right) ; 6.54\left(\mathrm{~d}, 1 \mathrm{H}, \mathrm{PhOPhOCH}_{3}, J=3.0 \mathrm{~Hz}\right) ; 5.02(\mathrm{dd}, 1 \mathrm{H}$, $\mathrm{PhCHO}, J=10.6$ and $2.3 \mathrm{~Hz}$ ); $4.18\left(\mathrm{dd}, 1 \mathrm{H}, \mathrm{CH}_{3} \mathrm{OPhCH}, J=5.3\right.$ and $3.5 \mathrm{~Hz}$ ); $3.82(\mathrm{~s}$, $\left.3 \mathrm{H}, \mathrm{OCH}_{3}\right) ; 3.71\left(\mathrm{~s}, 3 \mathrm{H}, \mathrm{OCH}_{3}\right) ; 2.43-2.48\left(\mathrm{~m}, 1 \mathrm{H}, \mathrm{CHCH}_{2}\right) .{ }^{13} \mathrm{C}\left\{{ }^{1} \mathrm{H}\right\} \mathrm{NMR} \delta 158.0$ (Quat.), 149.4 (Quat.), 138.1 (Quat.), 129.5 (Arom. CH), 128.4 (Arom. CH), 127.7 (Arom. CH), 126.0 (Arom. CH), 123.7 (Quat.), 117.6 (Arom. CH), 114.64 (Arom. CH), 114.56 (Arom. CH), 113.7 (Arom. $\mathrm{CH}), 73.0$ (PhCHO), $55.6\left(\mathrm{OCH}_{3}\right), 39.7\left(\mathrm{ArCHCH}_{2}\right)$, $38.5\left(\mathrm{ArCHCH}_{2}\right)$.

Spectra of $\mathbf{4 f}$ from $\mathrm{Et}_{3} \mathrm{SiH} / \mathrm{BF}_{3} \cdot \mathrm{Et}_{2} \mathrm{O}$ reduction 


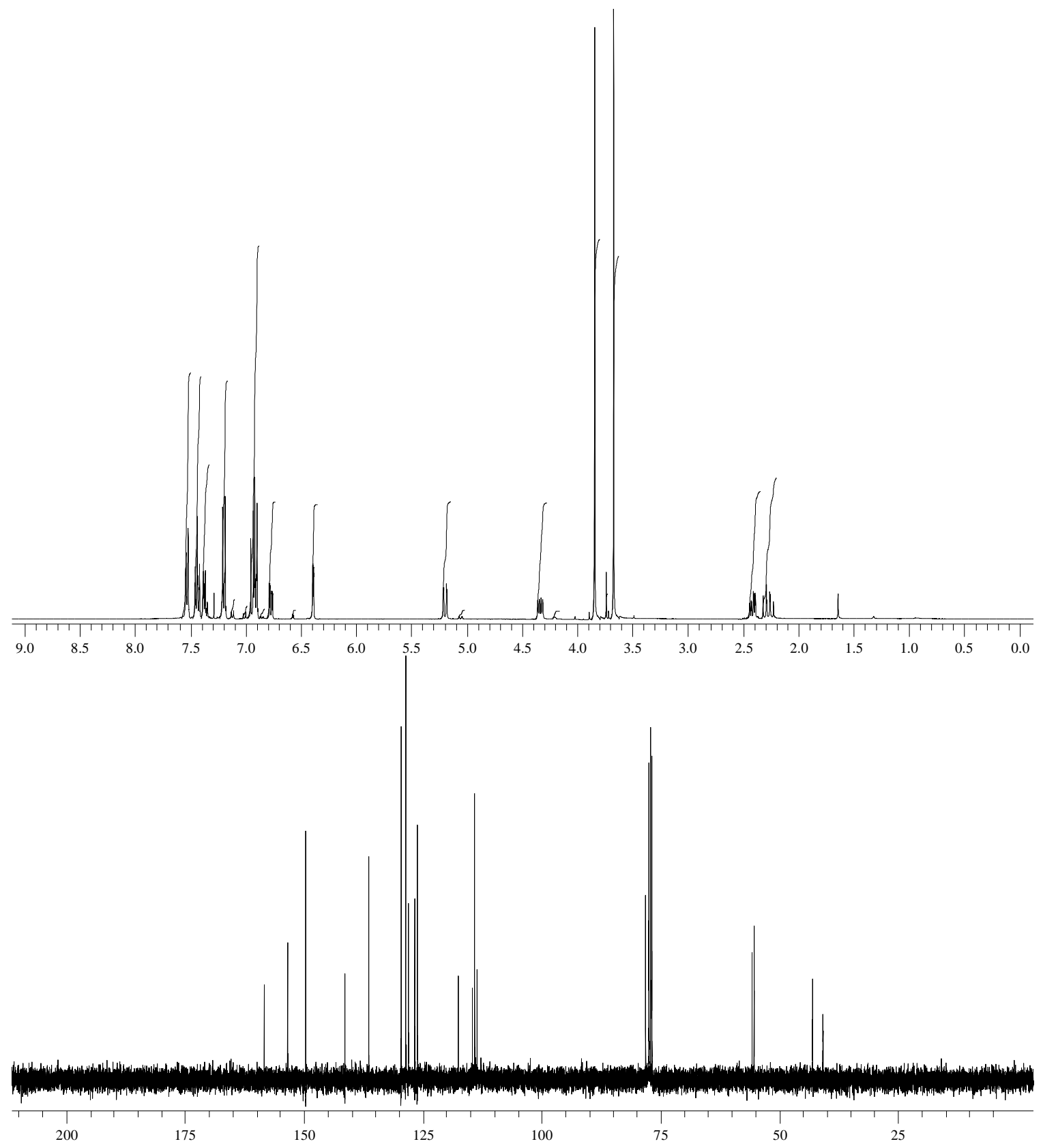

Spectra of $\mathbf{4 f}$ from $\mathrm{PhSiH}_{3} / \mathrm{BF}_{3} \cdot \mathrm{Et}_{2} \mathrm{O}$ reduction 

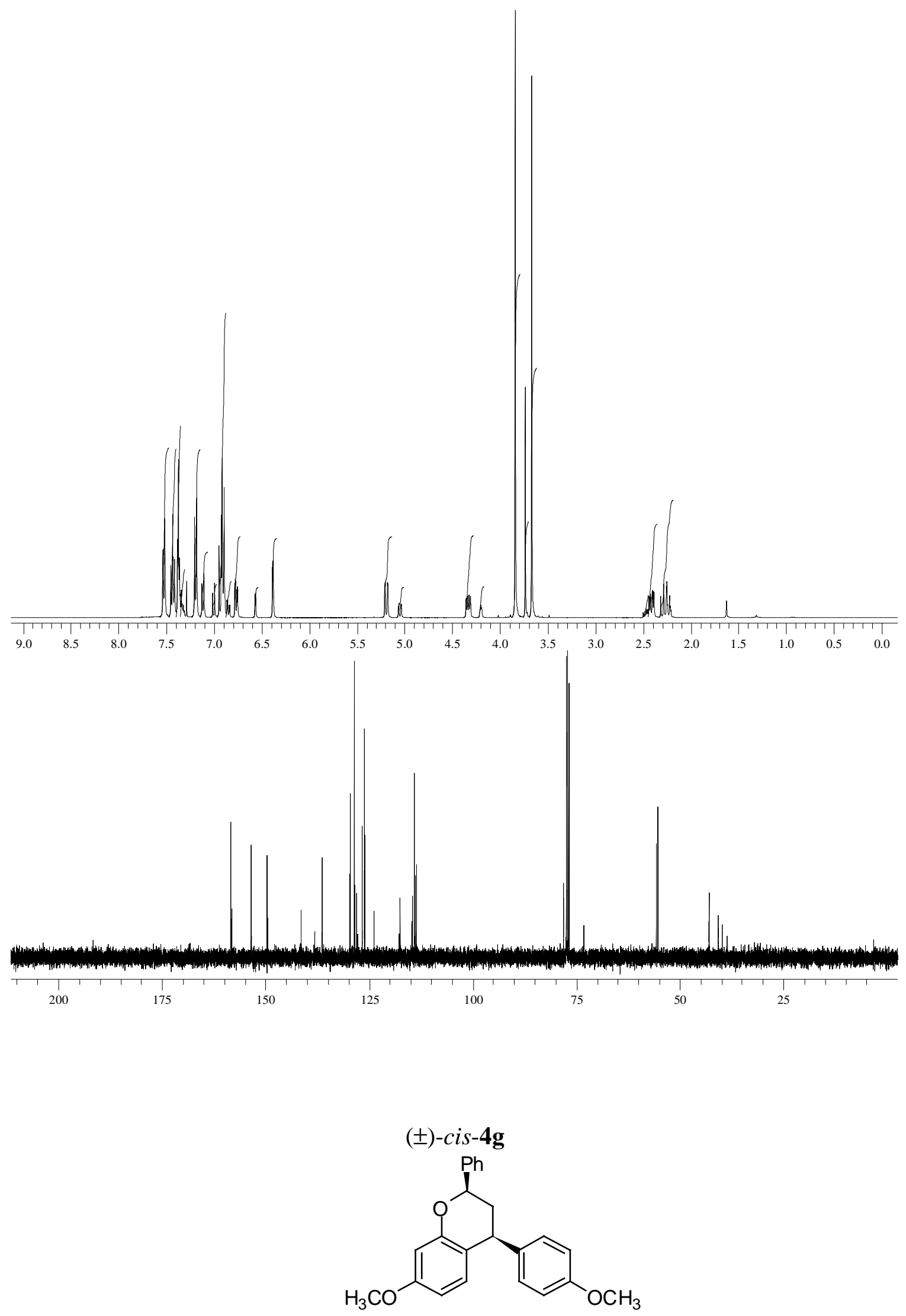

S-46 
Column chromatography on silica gel $\left(\mathrm{DCM} / \mathrm{hexane}=1 / 1, \mathrm{R}_{\mathrm{f}}=0.4\right)$ to afford $4 \mathrm{~g}$.

( \pm )-cis-7-methoxy-4-(4-methoxyphenyl)-2-phenylchroman . ${ }^{1} \mathrm{H}$ NMR $\left(\mathrm{CDCl}_{3}\right) \delta 7.50$ (d, $2 \mathrm{H}, P h \mathrm{CHO}, J=7.3 \mathrm{~Hz}) ; 7.42(\mathrm{t}, 2 \mathrm{H}, P h \mathrm{CHO}, J=7.3 \mathrm{~Hz}) ; 7.35(\mathrm{t}, 1 \mathrm{H}, P h \mathrm{CHO}, J=7.3$ $\mathrm{Hz}) ; 7.16\left(\mathrm{~d}, 2 \mathrm{H}, P h \mathrm{OCH}_{3}, J=8.7 \mathrm{~Hz}\right) ; 6.88\left(\mathrm{~d}, 2 \mathrm{H}, P h \mathrm{OCH}_{3}, J=8.7 \mathrm{~Hz}\right) ; 6.70(\mathrm{~d}, 1 \mathrm{H}$, $\left.\mathrm{PhOPhOCH}_{3}, J=8.6 \mathrm{~Hz}\right) ; 6.53\left(\mathrm{~d}, 1 \mathrm{H}, \mathrm{PhOPhOCH}_{3}, J=2.6 \mathrm{~Hz}\right) ; 6.43(\mathrm{dd}, 1 \mathrm{H}$, $\mathrm{PhOPhOCH}_{3}, J=8.6$ and $\left.2.6 \mathrm{~Hz}\right) ; 5.21(\mathrm{dd}, 1 \mathrm{H}, \mathrm{PhCHO}, J=11.4$ and $1.8 \mathrm{~Hz}) ; 4.27$ (dd, $1 \mathrm{H}, \mathrm{CH}_{3} \mathrm{OPhCH}, J=12.1$ and $\left.5.8 \mathrm{~Hz}\right) ; 3.82\left(\mathrm{~s}, 3 \mathrm{H}, \mathrm{OCH}_{3}\right) ; 3.78\left(\mathrm{~s}, 3 \mathrm{H}, \mathrm{OCH}_{3}\right) ; 2.38$ (ddd, $1 \mathrm{H}, \mathrm{CHCH}_{2}, J=13.6,5.8$ and $\left.2.0 \mathrm{~Hz}\right) ; 2.19-2.29\left(\mathrm{~m}, 1 \mathrm{H}, \mathrm{CHCH}_{2}\right) .{ }^{13} \mathrm{C}\left\{{ }^{1} \mathrm{H}\right\} \mathrm{NMR}$

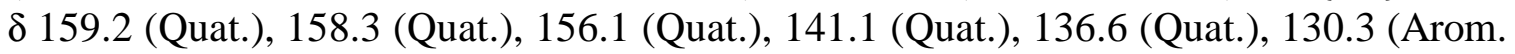
$\mathrm{CH}$ ), 129.3 (Arom. $\mathrm{CH}$ ), 128.5 (Arom. $\mathrm{CH}$ ), 128.0 (Arom. $\mathrm{CH}$ ), 126.1 (Arom. $\mathrm{CH}$ ), 118.2 (Quat.), 113.9 (Arom. CH), 107.6 (Arom. CH), 101.3 (Arom. CH), 78.4 (PhCHO), $55.23\left(\mathrm{OCH}_{3}\right), 55.20\left(\mathrm{OCH}_{3}\right), 42.0\left(\mathrm{ArCHCH}_{2}\right), 40.7\left(\mathrm{ArCHCH}_{2}\right) . \quad v_{\max }(\mathrm{NaCl}) / \mathrm{cm}^{-1}$ : 3006, 1616, 1579, 1502, 1442, 1271, 1249, 1157, 1176, 1033. HR-MS: $\mathrm{C}_{23} \mathrm{H}_{23} \mathrm{O}_{3}$ Calcd. $347.1647(\mathrm{M}+\mathrm{H})$; found: 347.1640 .

\section{(士)-trans-4g}

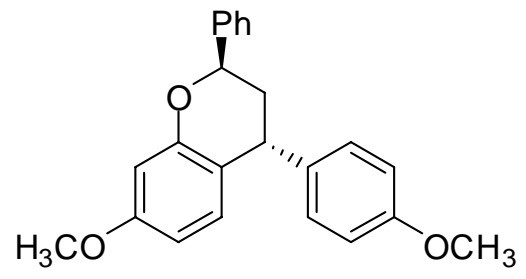

(士)-trans-4-(4-methoxyphenyl)-5,7-dimethyl-2-phenylchroman . ${ }^{1} \mathrm{H} \mathrm{NMR}\left(\mathrm{CDCl}_{3}\right) \delta 7.08$ $\left(\mathrm{d}, 2 \mathrm{H}, P h \mathrm{OCH}_{3}, J=8.6 \mathrm{~Hz}\right) ; 6.62\left(\mathrm{~d}, 1 \mathrm{H}, \mathrm{PhOPhOCH}_{3}, J=2.6 \mathrm{~Hz}\right) ; 5.05(\mathrm{dd}, 1 \mathrm{H}$, $P h \mathrm{CHO}, J=10.6$ and $2.5 \mathrm{~Hz}) ; 4.16\left(\mathrm{dd}, 1 \mathrm{H}, \mathrm{CH}_{3} \mathrm{OPhCH}, J=4.8\right.$ and $\left.3.3 \mathrm{~Hz}\right), 2.42-2.48$ (m, $1 \mathrm{H}, \mathrm{CHCH}_{2}$ ). ${ }^{13} \mathrm{C}\left\{{ }^{1} \mathrm{H}\right\}$ NMR $\delta 128.4$ (Arom. CH), 126.0 (Arom. $\mathrm{CH}$ ), 113.7 (Arom. $\mathrm{CH}$ ). Since only minor quantities were formed, only a partial spectra could be identified. 


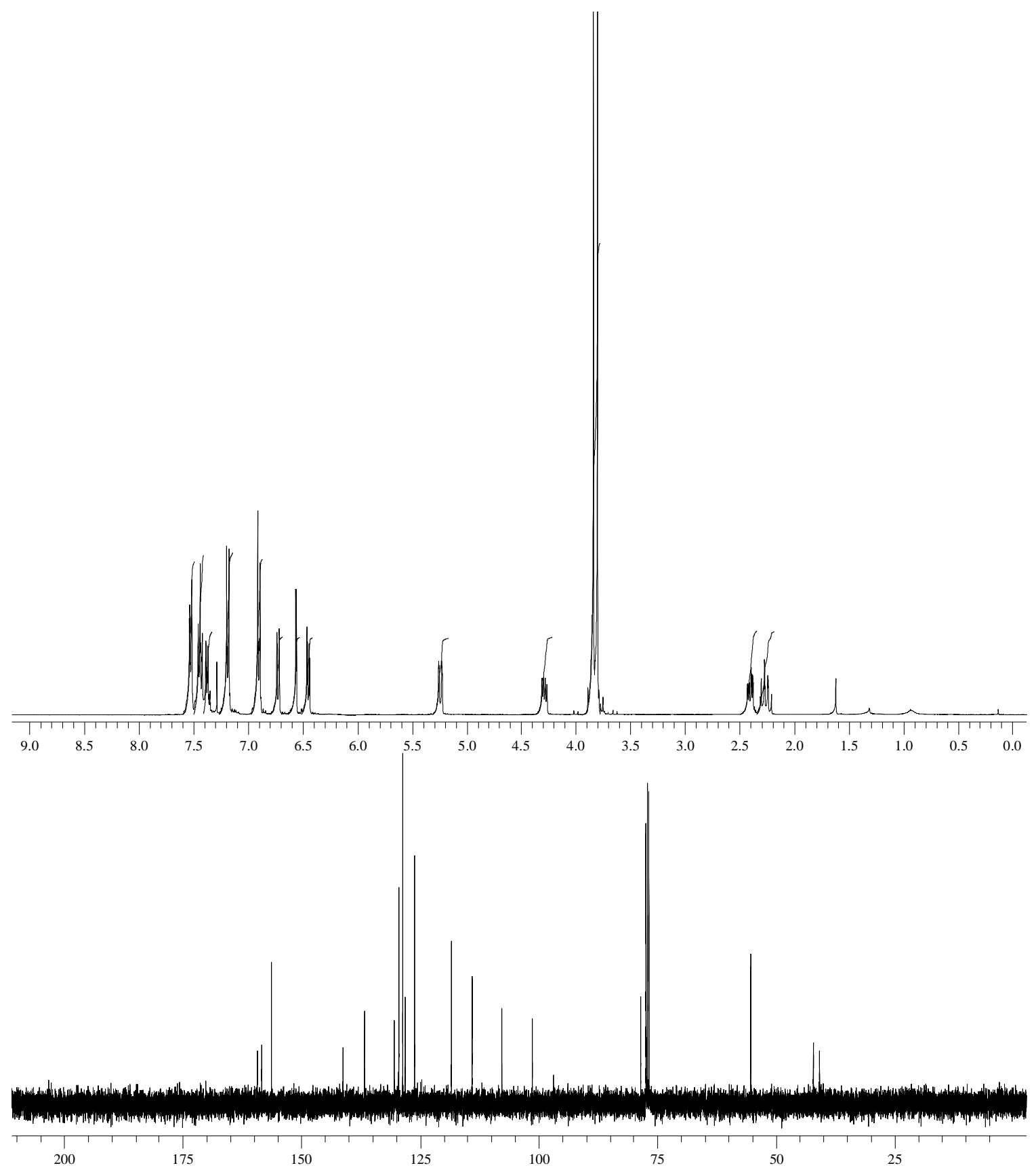

Spectra of $\mathbf{4} \mathbf{g}$ from $\mathrm{PhSiH}_{3} / \mathrm{BF}_{3} \cdot \mathrm{Et}_{2} \mathrm{O}$ reduction 


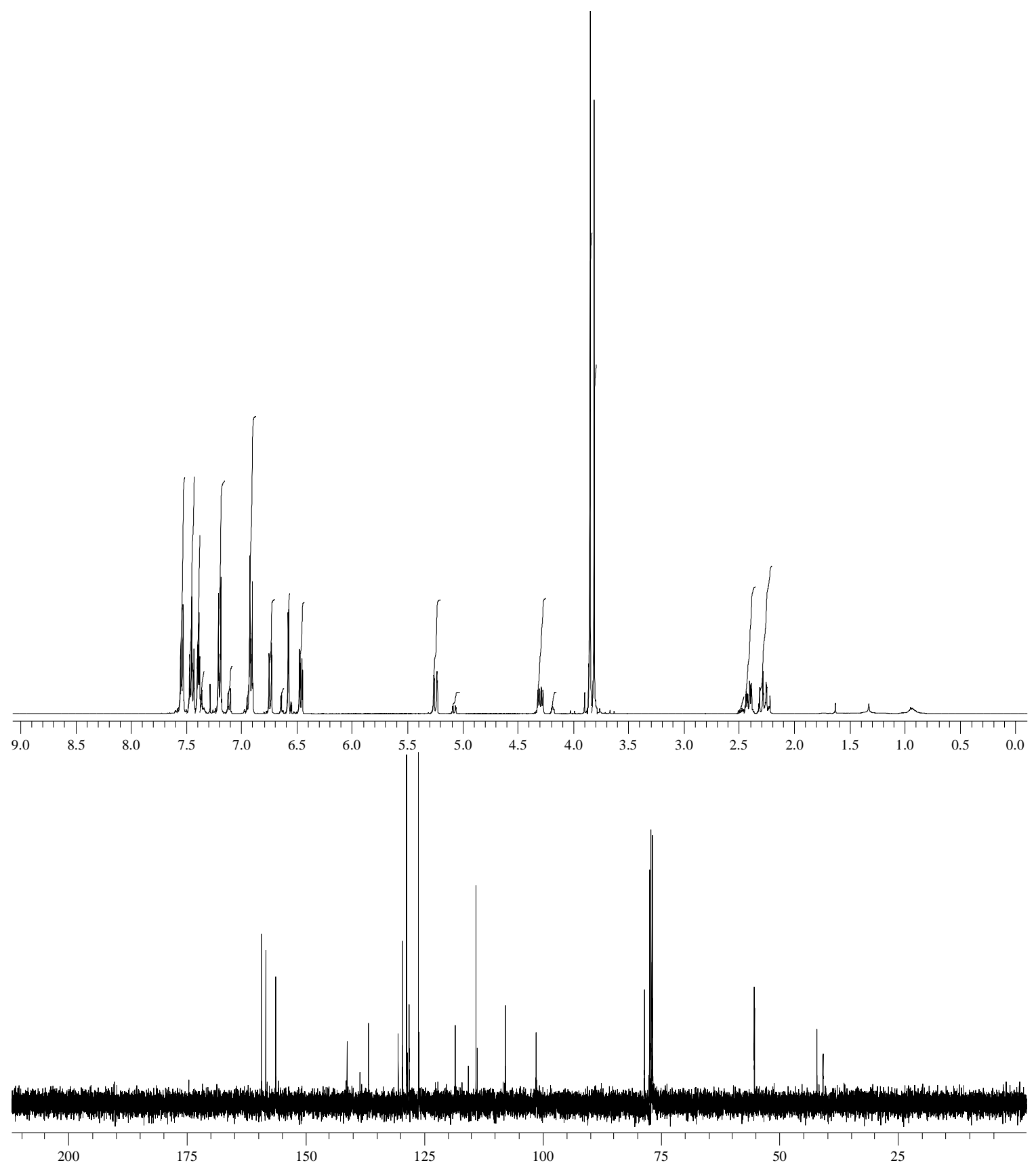

(士)-cis-4h<smiles>COc1ccc(C2CC(c3ccccc3)Oc3cc(C)cc(C)c32)cc1</smiles> 
Column chromatography on silica gel $\left(\mathrm{DCM} /\right.$ hexane $\left.=1 / 1, \mathrm{R}_{\mathrm{f}}=0.7\right)$ to afford $4 \mathbf{h}$.

( \pm )-cis-4-(4-methoxyphenyl)-5,7-dimethyl-2-phenylchroman . ${ }^{1} \mathrm{H}$ NMR $\left(\mathrm{CDCl}_{3}\right) \delta 7.44$ $(\mathrm{d}, 2 \mathrm{H}, P h \mathrm{CHO}, J=7.1 \mathrm{~Hz}$ ); 7.37 (t, $2 \mathrm{H}, P h \mathrm{CHO}, J=7.1 \mathrm{~Hz}$ ); 7.30 (t, $1 \mathrm{H}, P h \mathrm{CHO}, J=$ $7.1 \mathrm{~Hz}) ; 7.01\left(\mathrm{~d}, 2 \mathrm{H}, P h \mathrm{OCH}_{3}, J=8.7 \mathrm{~Hz}\right) ; 6.79\left(\mathrm{~d}, 2 \mathrm{H}, P h \mathrm{OCH}_{3}, J=8.7 \mathrm{~Hz}\right) ; 6.73(\mathrm{~s}$, $\left.1 \mathrm{H}, P h \mathrm{CH}_{3}\right) ; 6.58\left(\mathrm{~s}, 1 \mathrm{H}, P h \mathrm{CH}_{3}\right) ; 4.98(\mathrm{dd}, 1 \mathrm{H}, \mathrm{PhCHO}, J=11.4$ and $1.3 \mathrm{~Hz}) ; 4.30(\mathrm{dd}$, $1 \mathrm{H}, \mathrm{CH}_{3} \mathrm{OPhCH}, J=10.6$ and $\left.8.1 \mathrm{~Hz}\right) ; 3.78\left(\mathrm{~s}, 3 \mathrm{H}, \mathrm{OCH}_{3}\right) ; 2.55-2.61\left(\mathrm{~m}, 1 \mathrm{H}, \mathrm{CHCH}_{2}\right)$; $2.29\left(\mathrm{~s}, 3 \mathrm{H}, \mathrm{PhCH}_{3}\right) ; 2.12-2.21\left(\mathrm{~m}, 1 \mathrm{H}, \mathrm{CHCH}_{2}\right) ; 1.79\left(\mathrm{~s}, 3 \mathrm{H}, \mathrm{PhCH} \mathrm{H}_{3}\right) .{ }^{13} \mathrm{C}\left\{{ }^{1} \mathrm{H}\right\} \mathrm{NMR} \delta$ 157.7 (Quat.), 157.0 (Quat.), 141.0 (Quat.), 138.6 (Quat.), 137.1 (Quat.), 128.4 (Quat.), 128.1 (Arom. CH), 127.8 (Arom. CH), 126.1 (Arom. CH), 124.7 (Arom. $\mathrm{CH}$ ), 121.2 (Quat.), 115.7 (Arom. CH), 114.0 (Arom. $\mathrm{CH}$ ), 77.4 ( $\mathrm{PhCHO}), 55.2\left(\mathrm{OCH}_{3}\right), 43.8$ $\left(\mathrm{ArCHCH} \mathrm{H}_{2}\right), 40.8\left(\mathrm{ArCHCH}_{2}\right), 21.0\left(\mathrm{ArCH}_{3}\right), 20.9\left(\mathrm{ArCH}_{3}\right) . v_{\max }(\mathrm{NaCl}) / \mathrm{cm}^{-1}: 3032$, 3007, 1610, 1571, 1510, 1301, 1247, 1176, 1072, 1033. HR-MS: $\mathrm{C}_{24} \mathrm{H}_{25} \mathrm{O}_{2}$ Calcd. $345.1854(\mathrm{M}+\mathrm{H})$; found: 345.1847 .

\section{(土)-trans-4h}

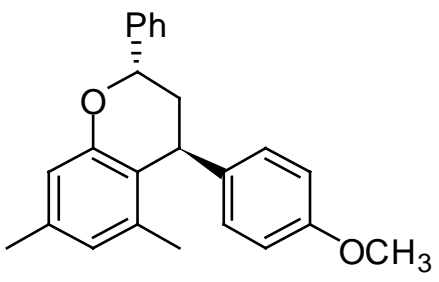

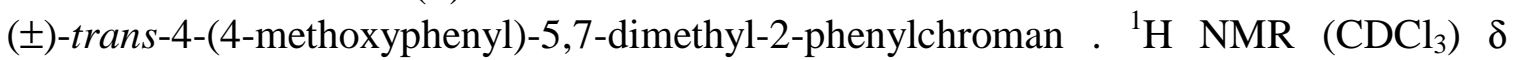
7.35-7.38 (m, 4H, PhCHO); 7.29-7.34 (m, 1H, PhCHO); 7.08 (d, 2H, $P h \mathrm{OCH}_{3}, J=8.8$ $\mathrm{Hz}) ; 6.79\left(\mathrm{~d}, 2 \mathrm{H}, P h \mathrm{OCH}_{3}, J=8.8 \mathrm{~Hz}\right) ; 6.79\left(\mathrm{~s}, 1 \mathrm{H}, P h \mathrm{CH}_{3}\right) ; 6.69\left(\mathrm{~s}, 1 \mathrm{H}, P h \mathrm{CH}_{3}\right) ; 4.93$ (dd, $1 \mathrm{H}, \mathrm{PhCHO}, J=11.9$ and $2.0 \mathrm{~Hz}) ; 4.24\left(\mathrm{~d}, \mathrm{br}, 1 \mathrm{H}, \mathrm{CH}_{3} \mathrm{OPhCH}, J=4.0 \mathrm{~Hz}\right) ; 3.84$ (s, $\left.3 \mathrm{H}, \mathrm{OCH}_{3}\right) ; 2.38-2.43\left(\mathrm{~m}, 1 \mathrm{H}, \mathrm{CHCH}_{2}\right) ; 2.36\left(\mathrm{~s}, 3 \mathrm{H}, \mathrm{PhCH}_{3}\right) ; 2.17-2.22(\mathrm{~m}, 1 \mathrm{H}$, $\left.\mathrm{CHCH}_{2}\right) ; 2.02\left(\mathrm{~s}, 3 \mathrm{H}, \mathrm{PhCH}_{3}\right) .{ }^{13} \mathrm{C}\left\{{ }^{1} \mathrm{H}\right\} \mathrm{NMR} \delta 157.9$ (Quat.), 155.3 (Quat.), 141.7 (Quat.), 137.8 (Quat.), 137.5 (Quat.), 137.3 (Quat.), 129.2 (Arom. CH), 128.3 (Arom. $\mathrm{CH}$ ), 127.7 (Arom. CH), 126.1 (Arom. CH), 123.4 (Arom. CH), 118.4 (Quat.), 115.1 (Arom. CH), 113.7 (Arom. CH), $72.2(\mathrm{PhCHO}), 55.1\left(\mathrm{OCH}_{3}\right), 39.2\left(\mathrm{ArCHCH}_{2}\right), 37.7$ $(\mathrm{ArCHCH}), 21.0\left(\mathrm{ArCH}_{3}\right), 18.9\left(\mathrm{ArCH}_{3}\right) . v_{\max }(\mathrm{NaCl}) / \mathrm{cm}^{-1}: 3032,3005,1610,1575$, 1508, 1454, 1303, 1247, 1145, 1089, 1034. HR-MS: $\mathrm{C}_{24} \mathrm{H}_{25} \mathrm{O}_{2}$ Calcd. $345.1854(\mathrm{M}+\mathrm{H})$; found: 345.1844 . 

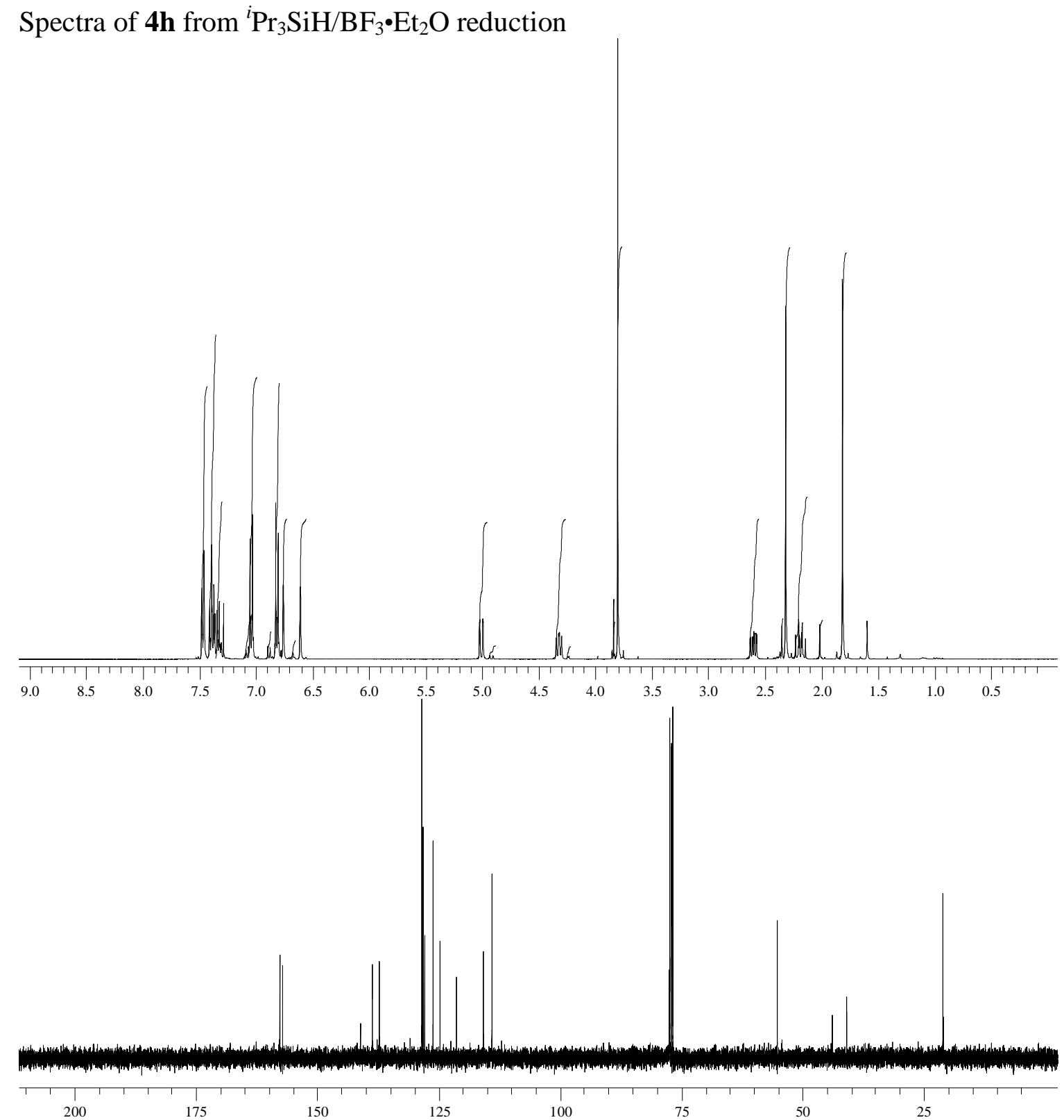
Spectra of $\mathbf{4 h}$ from $\mathrm{PhSiH}_{3} / \mathrm{BF}_{3} \cdot \mathrm{Et}_{2} \mathrm{O}$ reduction

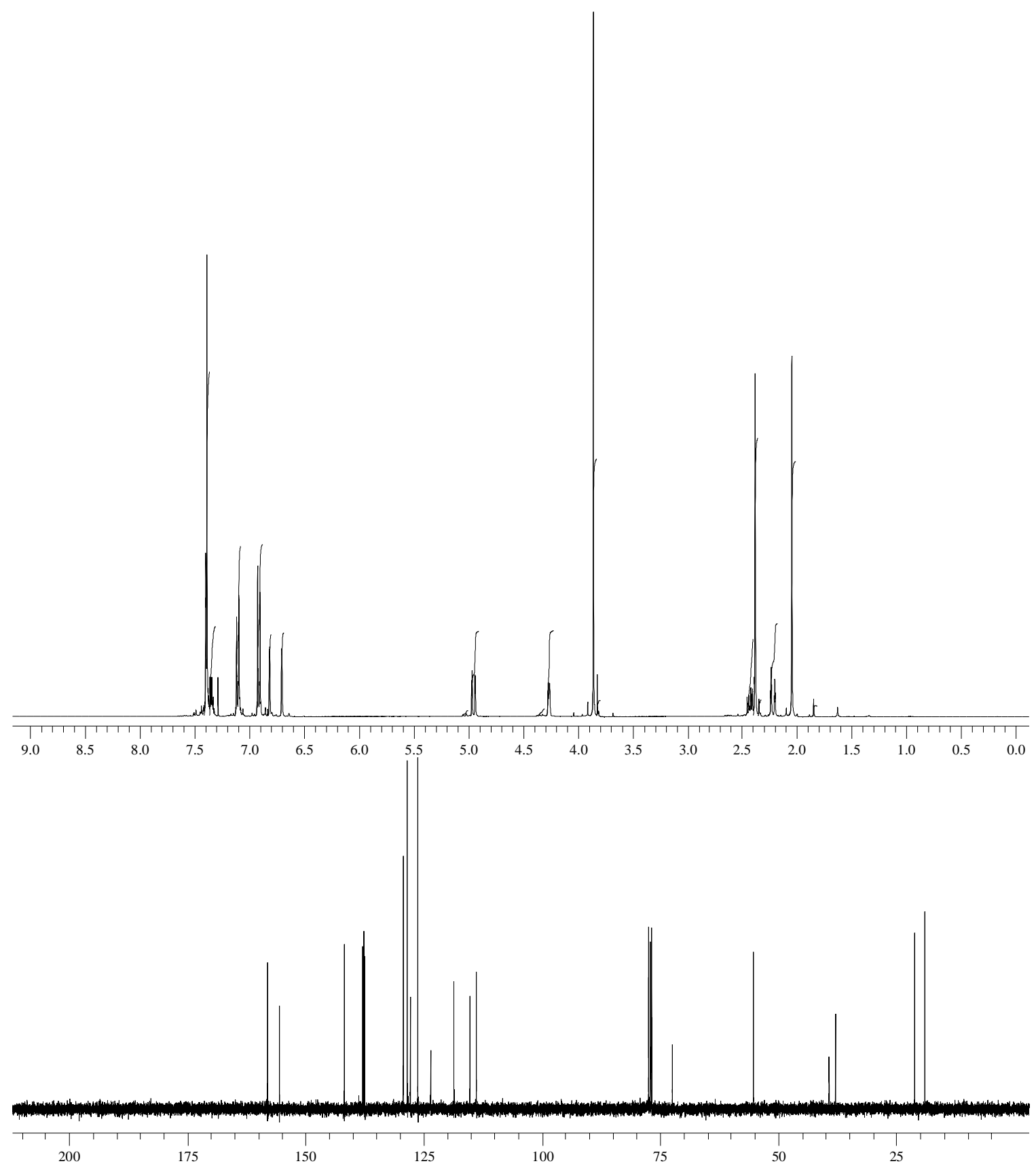




\section{$( \pm)-c i s-4 \mathbf{i}$}

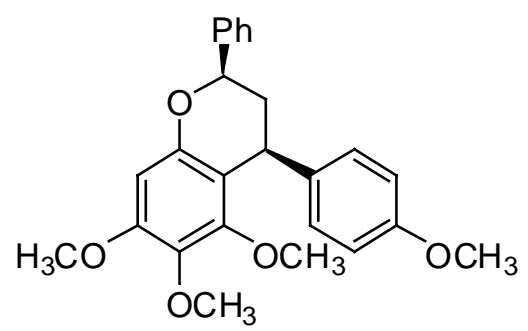

Column chromatography on silica gel $\left(\mathrm{DCM}, \mathrm{R}_{\mathrm{f}}=0.4\right)$ to afford $4 \mathbf{i}$.

( \pm )-cis-5,6,7-trimethoxy-4-(4-methoxyphenyl)-2-phenylchroman. ${ }^{1} \mathrm{H}$ NMR $\left(\mathrm{CDCl}_{3}\right) \delta$ $7.45(\mathrm{~d}, 2 \mathrm{H}, P h \mathrm{CHO}, J=7.1 \mathrm{~Hz}) ; 7.38(\mathrm{t}, 2 \mathrm{H}, P h \mathrm{CHO}, J=7.1 \mathrm{~Hz}) ; 7.31(\mathrm{t}, 1 \mathrm{H}, P h \mathrm{CHO}$, $J=7.1 \mathrm{~Hz}) ; 7.11\left(\mathrm{~d}, 2 \mathrm{H}, P h \mathrm{OCH}_{3}, J=8.7 \mathrm{~Hz}\right) ; 6.82\left(\mathrm{~d}, 2 \mathrm{H}, P h \mathrm{OCH}_{3}, J=8.7 \mathrm{~Hz}\right) ; 6.37$ $\left(\mathrm{s}, 1 \mathrm{H}, \mathrm{CH}_{3} \mathrm{OPh}\right) ; 4.93(\mathrm{dd}, 1 \mathrm{H}, \mathrm{PhCHO}, J=11.6$ and $1.3 \mathrm{~Hz}) ; 4.28(\mathrm{dd}, 1 \mathrm{H}$, $\mathrm{CH}_{3} \mathrm{OPhCH}, J=11.1$ and $\left.7.6 \mathrm{~Hz}\right) ; 3.83\left(\mathrm{~s}, 3 \mathrm{H}, \mathrm{OCH}_{3}\right) ; 3.78\left(\mathrm{~s}, 3 \mathrm{H}, \mathrm{OCH}_{3}\right) ; 3.73(\mathrm{~s}, 3 \mathrm{H}$, $\left.\mathrm{OCH}_{3}\right) ; 3.15\left(\mathrm{~s}, 3 \mathrm{H}, \mathrm{OCH}_{3}\right) ; 2.45-2.51\left(\mathrm{~m}, 1 \mathrm{H}, \mathrm{CHCH}_{2}\right) ; 2.05-2.15\left(\mathrm{~m}, 1 \mathrm{H}, \mathrm{CHCH}_{2}\right)$. ${ }^{13} \mathrm{C}\left\{{ }^{1} \mathrm{H}\right\}$ NMR $\delta 157.7$ (Quat.), 152.83 (Quat.), 152.76 (Quat.), 152.3 (Quat.), 140.7 (Quat.), 139.7 (Quat.), 136.7 (Quat.), 128.5 (Arom. CH), 128.01 (Arom. CH), 128.0 (Arom. CH), 126.1 (Arom. CH), 113.6 (Arom. CH), 112.1 (Quat.), 96.2 (Arom. CH), $78.0(\mathrm{PhCHO}), 60.6\left(\mathrm{OCH}_{3}\right), 59.4\left(\mathrm{OCH}_{3}\right), 55.7\left(\mathrm{OCH}_{3}\right), 55.2\left(\mathrm{OCH}_{3}\right), 42.6\left(\mathrm{ArCHCH}_{2}\right)$, $39.1\left(\mathrm{ArCHCH}_{2}\right) . v_{\max }(\mathrm{NaCl}) / \mathrm{cm}^{-1}: 3032,3003,1609,1582,1510,1485,1458,1409$, 1248, 1132, 1101, 1061, 1010. HR-MS: $\mathrm{C}_{25} \mathrm{H}_{26} \mathrm{O} \mathrm{O}_{5} \mathrm{Na}$ Calcd. 429.1678 (M+Na); found: 429.1667.

\section{$( \pm)-$ trans $-\mathbf{4 i}$}

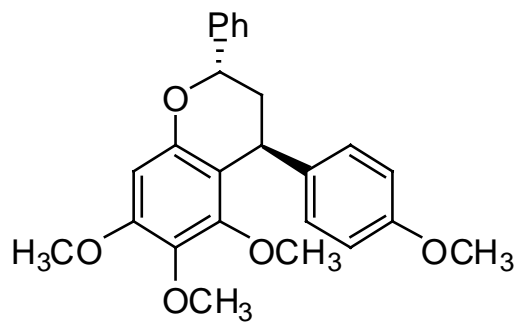

( \pm )-trans-5,6,7-trimethoxy-4-(4-methoxyphenyl)-2-phenylchroman. ${ }^{1} \mathrm{H}$ NMR $\left(\mathrm{CDCl}_{3}\right) \delta$ 7.28-7.34 (m, 5H, $P h \mathrm{CHO}$ ); 7.09 (d, $2 \mathrm{H}, P h \mathrm{OCH}_{3}, J=8.6 \mathrm{~Hz}$ ); 6.86 (d, $2 \mathrm{H}, P h \mathrm{OCH}_{3}, J$ $=8.7 \mathrm{~Hz}) ; 6.40\left(\mathrm{~s}, 1 \mathrm{H}, \mathrm{CH}_{3} \mathrm{OPh}\right) ; 4.92(\mathrm{dd}, 1 \mathrm{H}, \mathrm{PhCHO}, J=11.9$ and $2.0 \mathrm{~Hz}) ; 4.38(\mathrm{dd}$, $1 \mathrm{H}, \mathrm{CH}_{3} \mathrm{OPhCH}, J=5.3$ and $\left.1.5 \mathrm{~Hz}\right) ; 3.86\left(\mathrm{~s}, 3 \mathrm{H}, \mathrm{OCH}_{3}\right) ; 3.81\left(\mathrm{~s}, 6 \mathrm{H}, \mathrm{OCH}_{3}\right) ; 3.52(\mathrm{~s}$, $\left.3 \mathrm{H}, \mathrm{OCH}_{3}\right) ; 2.27-2.35\left(\mathrm{~m}, 1 \mathrm{H}, \mathrm{CHCH}_{2}\right) ; 2.06-2.15\left(\mathrm{~m}, 1 \mathrm{H}, \mathrm{CHCH}_{2}\right) .{ }^{13} \mathrm{C}\left\{{ }^{1} \mathrm{H}\right\} \mathrm{NMR} \delta$ 157.9 (Quat.), 151.6 (Quat.), 141.3 (Quat.), 138.7 (Quat.), 129.0 (Arom.CH), 128.5 (Arom.CH), 128.4 (Arom.CH), 128.1 (Quat.), 127.9 (Quat.), 126.2 (Arom.CH), 126.1 (Quat.), 113.5 (Arom.CH), 109.2 (Quat.), 95.9 (Arom.CH), 72.9 (PhCHO), $60.8\left(\mathrm{OCH}_{3}\right)$, $60.4\left(\mathrm{OCH}_{3}\right), 55.8\left(\mathrm{OCH}_{3}\right), 55.2\left(\mathrm{OCH}_{3}\right), 38.4\left(\mathrm{ArCHCH}_{2}\right), 35.2\left(\mathrm{ArCHCH}_{2}\right) . v \max$ $(\mathrm{NaCl}) / \mathrm{cm}^{-1}: 3032,3003,1610,1583,1510,1487,1460,1409,1245,1136,1103,1024$. HR-MS: $\mathrm{C}_{25} \mathrm{H}_{26} \mathrm{O}_{5} \mathrm{Na}$ Calcd. 429.1678 (M+Na); found: 429.1670. 


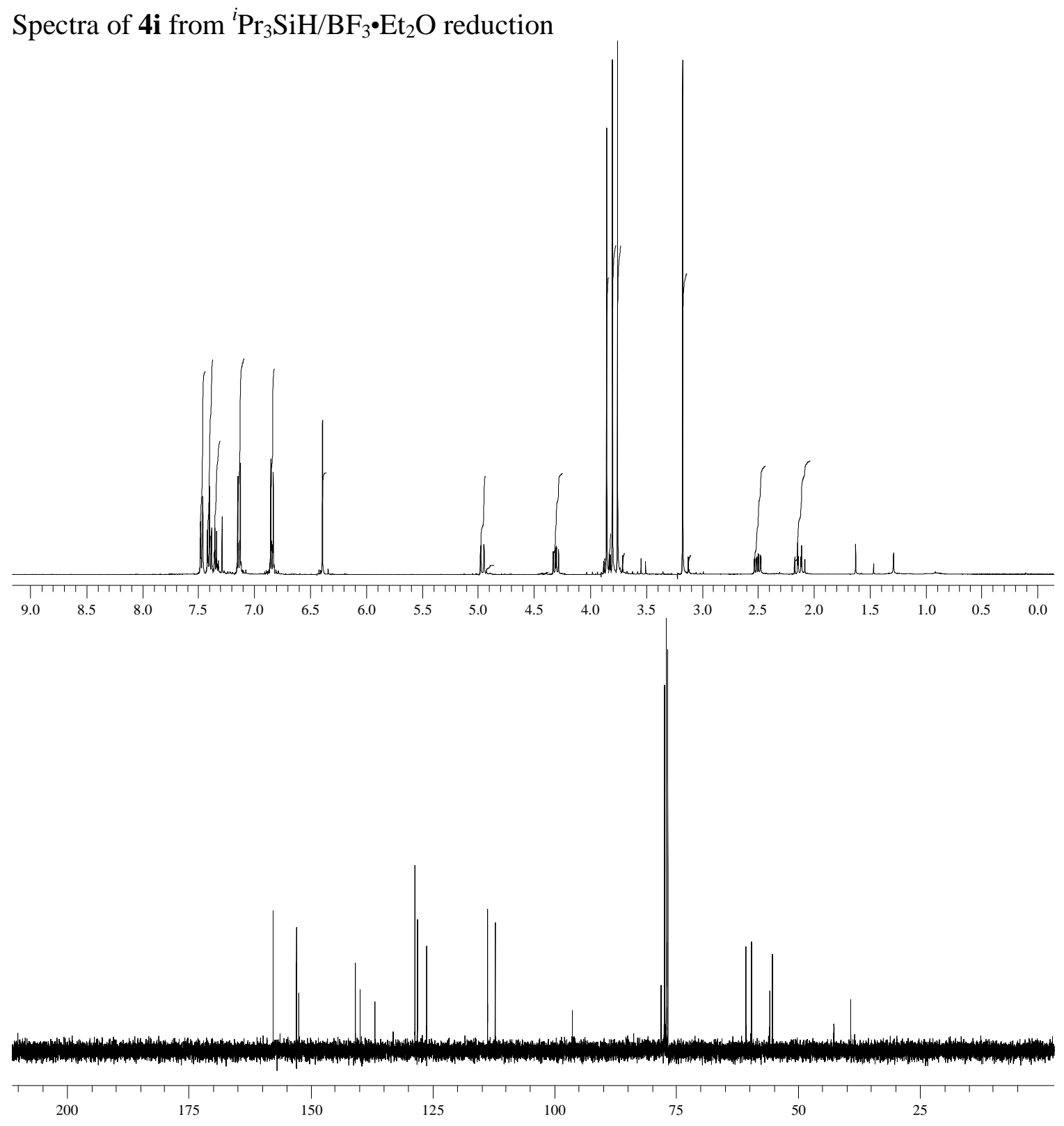




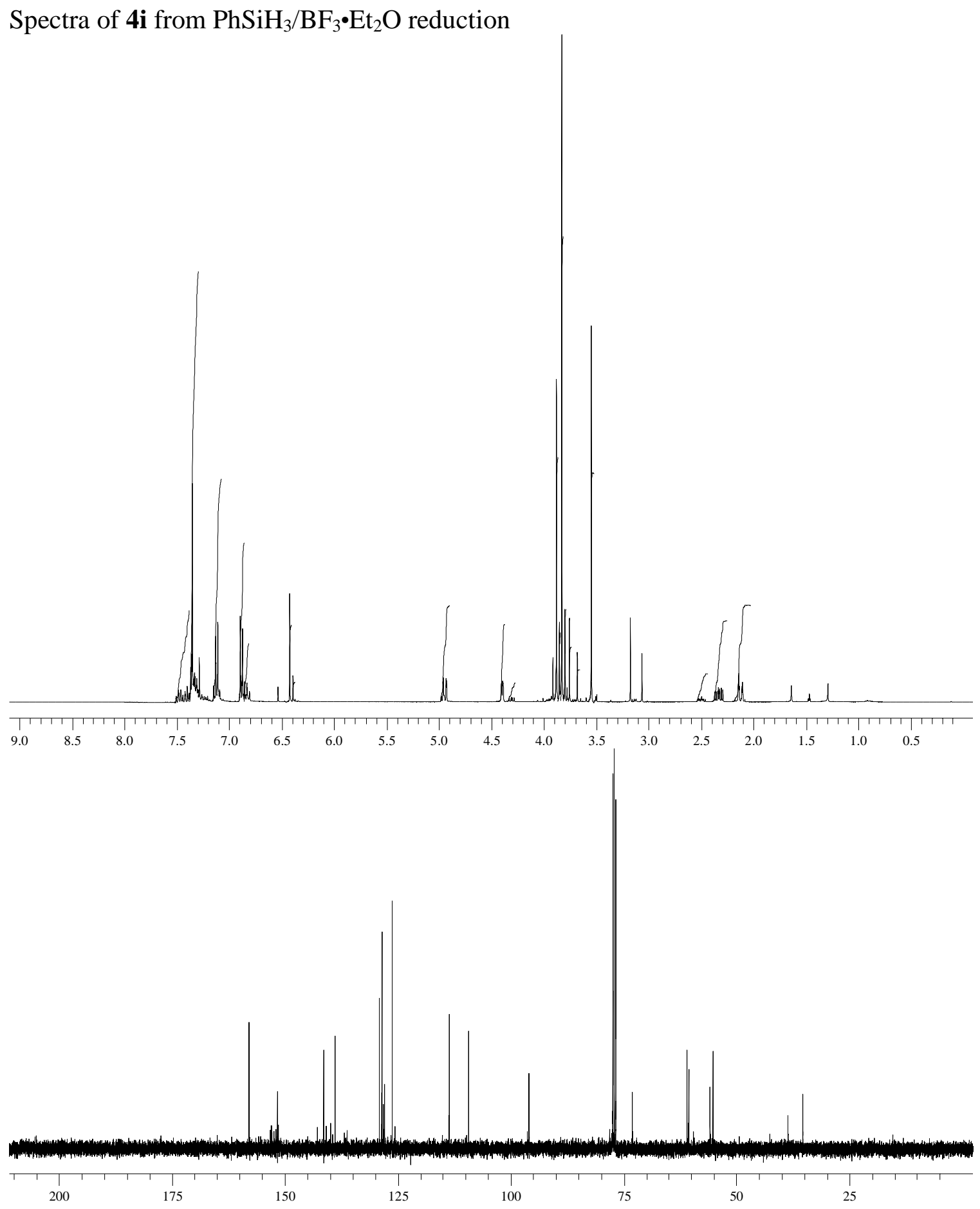

S-55 


\section{$( \pm)$-cis-4j}

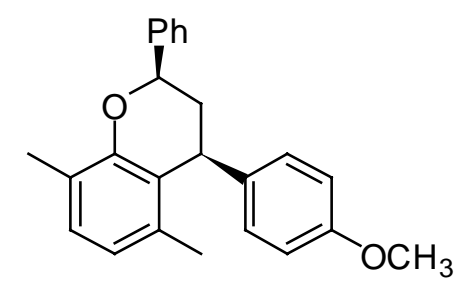

Column chromatography on silica gel (DCM/hexane $\left.=1 / 1, \mathrm{R}_{\mathrm{f}}=0.6\right)$ to afford $4 \mathbf{j}$.

( \pm )-cis-4-(4-Methoxyphenyl)-5,8-dimethyl-2-phenylchroman. ${ }^{1} \mathrm{H}$ NMR $\left(\mathrm{CDCl}_{3}\right) \delta 7.49$ (d, $2 \mathrm{H}, P h \mathrm{CHO}, J=7.3 \mathrm{~Hz}$ ); 7.39 (t, $2 \mathrm{H}, P h \mathrm{CHO}, J=7.3 \mathrm{~Hz}$ ); 7.31 (t, $1 \mathrm{H}, P h \mathrm{CHO}, J=$ $7.3 \mathrm{~Hz}) ; 7.01\left(\mathrm{~d}, 2 \mathrm{H}, P h \mathrm{OCH}_{3}, J=8.7 \mathrm{~Hz}\right) ; 7.00\left(\mathrm{~d}, 1 \mathrm{H}, P h \mathrm{CH}_{3}, J=7.6 \mathrm{~Hz}\right) ; 6.79(\mathrm{~d}, 2 \mathrm{H}$, $\left.P h \mathrm{OCH}_{3}, J=8.7 \mathrm{~Hz}\right) ; 6.66\left(\mathrm{~d}, 1 \mathrm{H}, P h \mathrm{CH}_{3}, J=7.6 \mathrm{~Hz}\right) ; 5.03(\mathrm{dd}, 1 \mathrm{H}, \mathrm{PhCHO}, J=11.4$ and $1.5 \mathrm{~Hz}) ; 4.36\left(\mathrm{dd}, 1 \mathrm{H}, \mathrm{CH}_{3} \mathrm{OPhCH}, J=10.6\right.$ and $\left.8.1 \mathrm{~Hz}\right) ; 3.78\left(\mathrm{~s}, 3 \mathrm{H}, \mathrm{OCH}_{3}\right) ; 2.65$ (ddd, $1 \mathrm{H}, \mathrm{CHCH}_{2}, J=13.9,7.8$ and $\left.1.8 \mathrm{~Hz}\right) ; 2.31\left(\mathrm{~s}, 3 \mathrm{H}, \mathrm{PhCH}_{3}\right) ; 2.10-2.19(\mathrm{~m}, 1 \mathrm{H}$, $\mathrm{CHCH}_{2}$ ); 1.81 (s, 3H, $\mathrm{PhCH}_{3}$ ). ${ }^{13} \mathrm{C}\left\{{ }^{1} \mathrm{H}\right\}$ NMR $\delta 157.6$ (Quat.), 155.1 (Quat.), 141.4 (Quat.), 138.7 (Quat.), 136.1 (Quat.), 128.4 (Arom. CH), 128.3 (Arom. CH), 128.2 (Arom. CH), 127.5 (Arom. CH), 125.8 (Arom. CH), 124.1 (Quat.), 123.8 (Quat.), 122.7 (Arom. $\mathrm{CH}$ ), 113.9 (Arom. $\mathrm{CH}), 76.8(\mathrm{PhCHO}), 55.2\left(\mathrm{OCH}_{3}\right), 43.8\left(\mathrm{ArCHCH}_{2}\right), 41.2$ $(\mathrm{ArCHCH}), 20.8\left(\mathrm{ArCH}_{3}\right), 16.1\left(\mathrm{ArCH}_{3}\right) . v_{\max }(\mathrm{NaCl}) / \mathrm{cm}^{-1}: 3032,3005,1609,1583$, 1508, 1483, 1462, 1302, 1222, 1176, 1033. HR-MS: $\mathrm{C}_{24} 4 \mathrm{H}_{28} \mathrm{O}_{2} \mathrm{~N}$ Calcd. 362.2120 $\left(\mathrm{M}+\mathrm{NH}_{4}\right)$; found: 362.2110 .

\section{$( \pm)-$ trans-4j}

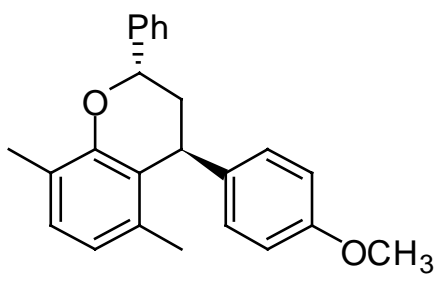

(士)-trans-4-(4-Methoxyphenyl)-5,8-dimethyl-2-phenylchroman. ${ }^{1} \mathrm{H}$ NMR $\left(\mathrm{CDCl}_{3}\right) \delta$ 7.37-7.41 (m, 4H, PhCHO); 7.29-7.33 (m, 1H, PhCHO); 7.07 (d, $2 \mathrm{H}, P h \mathrm{OCH}_{3}, J=8.7$ $\mathrm{Hz}) ; 7.06\left(\mathrm{~d}, 1 \mathrm{H}, P h \mathrm{CH}_{3}, J=7.6 \mathrm{~Hz}\right) ; 6.88\left(\mathrm{~d}, 2 \mathrm{H}, P h \mathrm{OCH}_{3}, J=8.7 \mathrm{~Hz}\right) ; 6.73(\mathrm{~d}, 1 \mathrm{H}$, $\left.P_{h C_{3}}, J=7.6 \mathrm{~Hz}\right) ; 4.96(\mathrm{dd}, 1 \mathrm{H}, \mathrm{PhCHO}, J=11.6$ and $2.5 \mathrm{~Hz}) ; 4.25(\mathrm{dd}, 1 \mathrm{H}$, $\mathrm{CH}_{3} \mathrm{OPhCH}, J=5.1$ and $\left.1.8 \mathrm{~Hz}\right) ; 3.83\left(\mathrm{~s}, 3 \mathrm{H}, \mathrm{OCH}_{3}\right) ; 2.31-2.35\left(\mathrm{~m}, 1 \mathrm{H}, \mathrm{CHCH}_{2}\right) ; 2.33$ $\left(\mathrm{s}, 3 \mathrm{H}, \mathrm{PhCH}_{3}\right) ; 2.20-2.25\left(\mathrm{~m}, 1 \mathrm{H}, \mathrm{CHCH}_{2}\right) ; 2.01\left(\mathrm{~s}, 3 \mathrm{H}, \mathrm{PhCH}_{3}\right) .{ }^{13} \mathrm{C}\left\{{ }^{1} \mathrm{H}\right\} \mathrm{NMR} \delta 157.9$ (Quat.), 153.4 (Quat.), 142.2 (Quat.), 137.4 (Quat.), 135.3 (Quat.), 129.3 (Arom. CH), 128.7 (Arom. CH), 128.3 (Arom. CH), 127.4 (Arom. CH), 125.8 (Arom. CH), 123.6 (Quat.), 121.4 (Arom. CH), 120.9 (Quat.), 113.8 (Arom. $\mathrm{CH}$ ), 71.9 (PhCHO), 55.2 $\left(\mathrm{OCH}_{3}\right), 39.3\left(\mathrm{ArCHCH}_{2}\right), 38.2\left(\mathrm{ArCHCH}_{2}\right), 18.8\left(\mathrm{ArCH}_{3}\right), 16.3\left(\mathrm{ArCH}_{3}\right) . \quad V_{\max }$ $(\mathrm{NaCl}) / \mathrm{cm}^{-1}: 3032,3005,1609,1583,1508,1485,1464,1442,1415,1302,1245,1178$, 1064, 1035. HR-MS: $\mathrm{C}_{24} \mathrm{H}_{25} \mathrm{O}_{2}$ Calcd. $345.1854(\mathrm{M}+\mathrm{H})$; found: 345.1855. 
Spectra of $\mathbf{4 j}$ from ${ }^{i} \mathrm{Pr}_{3} \mathrm{SiH}_{3} / \mathrm{BF}_{3} \cdot \mathrm{Et}_{2} \mathrm{O}$ reduction

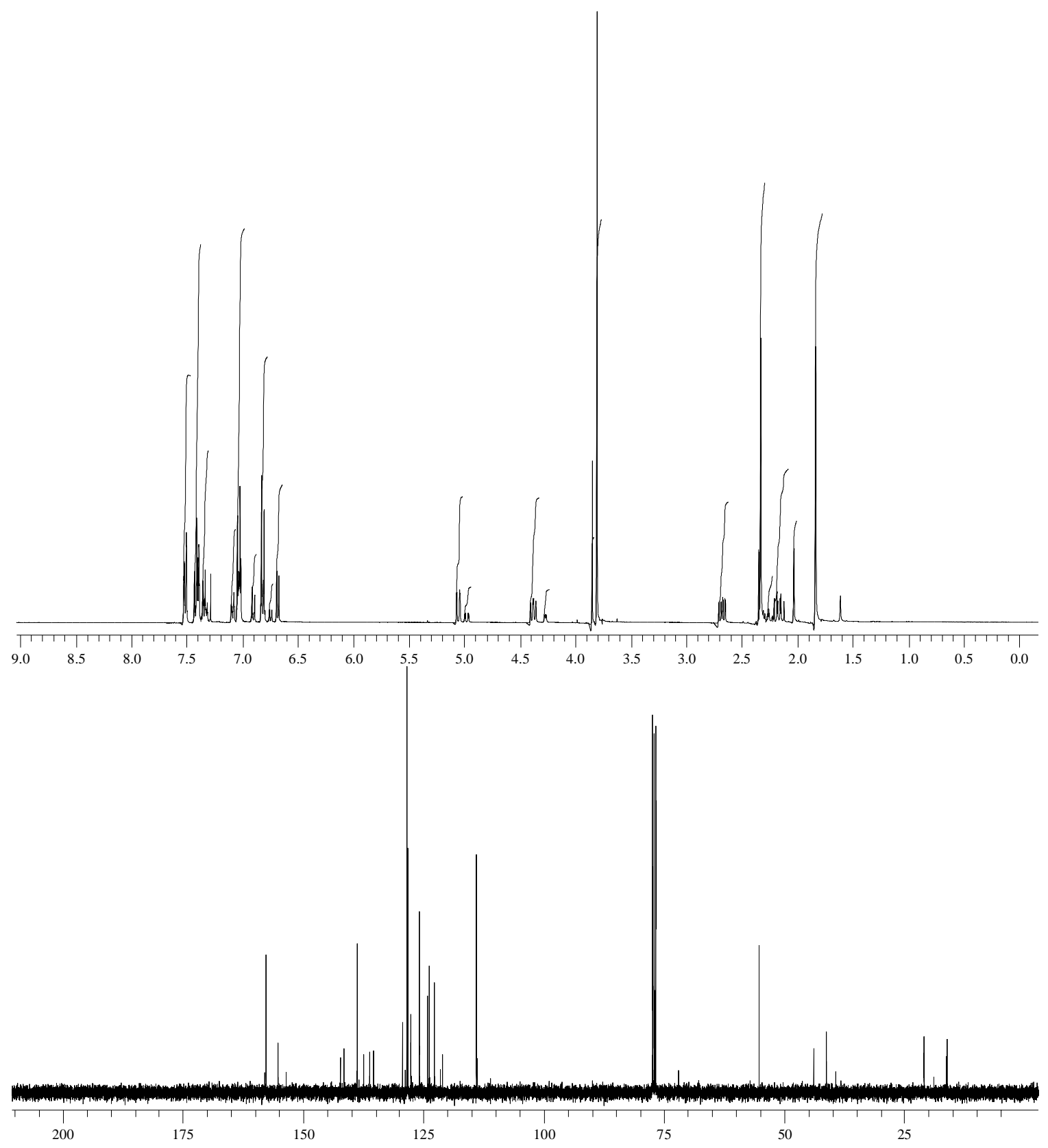


Spectra of $\mathbf{4 j}$ from $\mathrm{PhSiH}_{3} / \mathrm{BF}_{3} \cdot \mathrm{Et}_{2} \mathrm{O}$ reduction

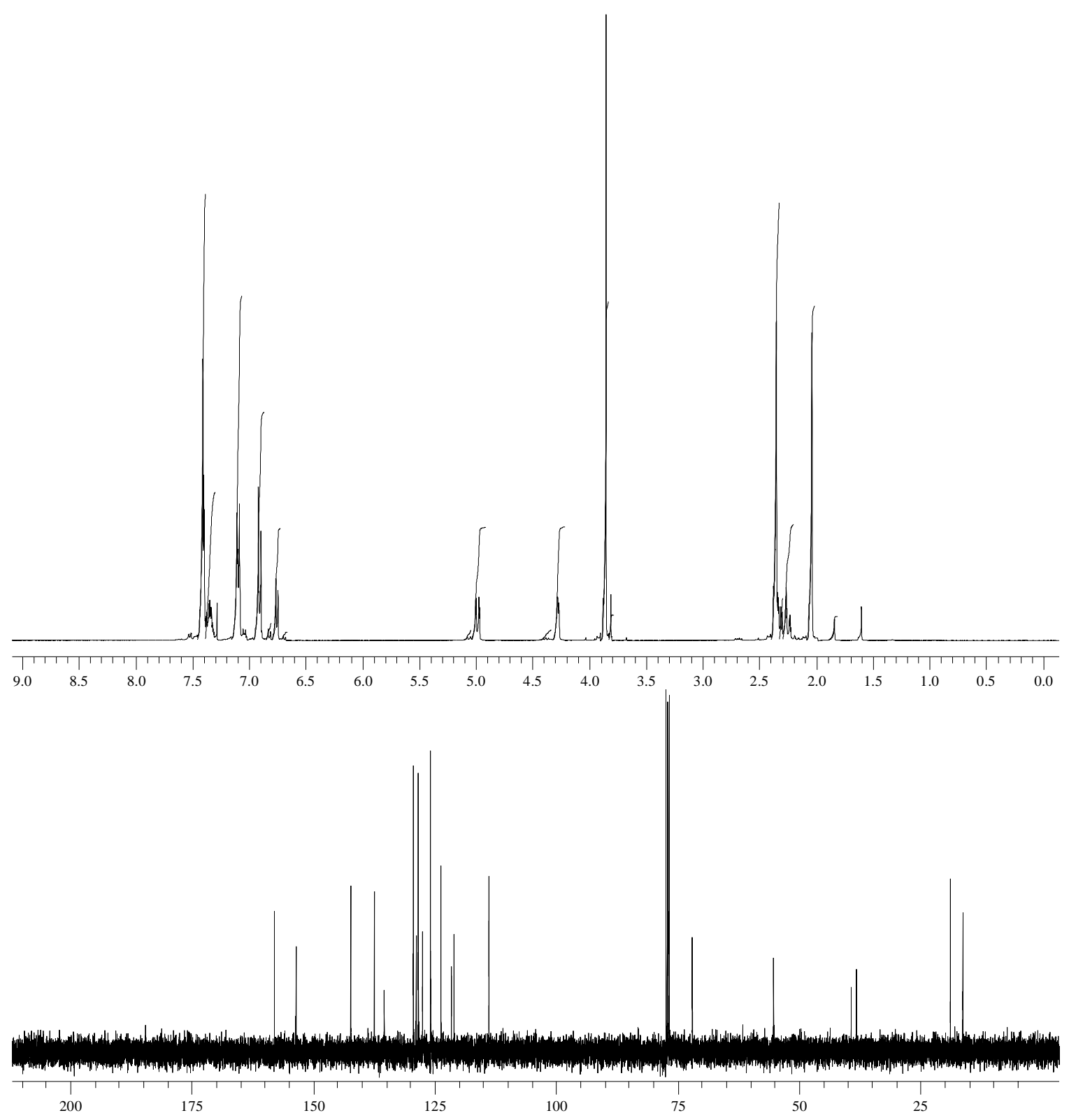


$( \pm)-c i s-4 \mathbf{k}$

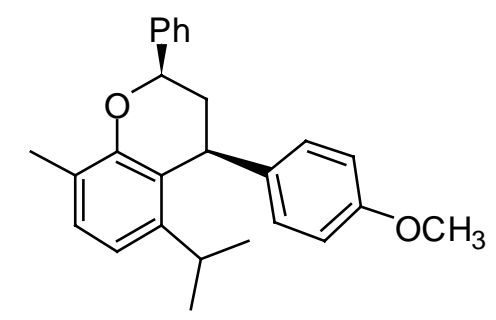

Column chromatography on silica gel $\left(\mathrm{DCM} /\right.$ hexane $\left.=1 / 1, \mathrm{R}_{\mathrm{f}}=0.6\right)$ to afford $4 \mathbf{k}$.

(士)-cis-5-Isopropyl-4-(4-methoxyphenyl)-8-methyl-2-phenylchroman. ${ }^{1} \mathrm{H} \mathrm{NMR}\left(\mathrm{CDCl}_{3}\right)$ $\delta 7.46(\mathrm{~d}, 2 \mathrm{H}, P h \mathrm{CHO}, J=7.3 \mathrm{~Hz}) ; 7.37(\mathrm{t}, 2 \mathrm{H}, P h \mathrm{CHO}, J=7.3 \mathrm{~Hz}) ; 7.29(\mathrm{t}, 1 \mathrm{H}$, $P h \mathrm{CHO}, J=7.3 \mathrm{~Hz}) ; 7.08\left(\mathrm{~d}, 1 \mathrm{H}, P h \mathrm{CH}_{3}, J=7.7 \mathrm{~Hz}\right) ; 6.97\left(\mathrm{~d}, 2 \mathrm{H}, P h \mathrm{OCH}_{3}, J=8.7\right.$ $\mathrm{Hz}) ; 6.80\left(\mathrm{~d}, 1 \mathrm{H}, P h \mathrm{CH}_{3}, J=7.7 \mathrm{~Hz}\right) ; 6.76\left(\mathrm{~d}, 2 \mathrm{H}, P h \mathrm{OCH}_{3}, J=8.7 \mathrm{~Hz}\right) ; 4.99$ (dd, 1H, $\mathrm{PhCHO}, J=11.1$ and $1.5 \mathrm{~Hz}) ; 4.49\left(\mathrm{dd}, 1 \mathrm{H}, \mathrm{CH}_{3} \mathrm{OPhCH}, J=9.9\right.$ and $\left.8.3 \mathrm{~Hz}\right) ; 3.77(\mathrm{~s}$, $\left.3 \mathrm{H}, \mathrm{OCH}_{3}\right) ; 2.80-2.87\left(\mathrm{~m}, 1 \mathrm{H}, \mathrm{CH}\left(\mathrm{CH}_{3}\right)_{2}\right) ; 2.68\left(\mathrm{ddd}, 1 \mathrm{H}, \mathrm{CHCH}_{2}, J=13.9,8.1\right.$ and 1.8 $\mathrm{Hz}) ; 2.30$ (s, 3H, $\left.\mathrm{PhCH}_{3}\right) ; 2.08-2.16\left(\mathrm{~m}, 1 \mathrm{H}, \mathrm{CHCH}_{2}\right) ; 1.09$ (d, 3H, $\mathrm{CH}\left(\mathrm{CH}_{3}\right)_{2}, J=6.8$ $\mathrm{Hz}$ ); 0.62 (d, 3H, $\left.\mathrm{CH}\left(\mathrm{CH}_{3}\right)_{2}, J=6.8 \mathrm{~Hz}\right) .{ }^{13} \mathrm{C}\left\{{ }^{1} \mathrm{H}\right\} \mathrm{NMR} \delta 157.6$ (Quat.), 155.0 (Quat.), 147.0 (Quat.), 141.4 (Quat.), 140.0 (Quat.), 128.9 (Arom. CH), 128.3 (Arom. CH), 128.2 (Arom. CH), 127.5 (Arom. CH), 125.8 (Arom. CH), 123.6 (Quat.), 122.6 (Quat.), 118.3 (Arom. CH), 113.8 (Arom. CH), $76.5(\mathrm{PhCHO}), 55.2\left(\mathrm{OCH}_{3}\right) 43.9\left(\mathrm{ArCHCH}_{2}\right), 40.2$ $(\mathrm{ArCHCH}), 29.1\left(\mathrm{CH}\left(\mathrm{CH}_{3}\right)_{2}\right), 25.0\left(\mathrm{CH}\left(\mathrm{CH}_{3}\right)_{2}\right), 22.3\left(\left(\mathrm{CH}\left(\mathrm{CH}_{3}\right)_{2}\right), 16.1\left(\mathrm{ArCH}_{3}\right) . v_{\max }\right.$ $(\mathrm{NaCl}) / \mathrm{cm}^{-1}: 3032,3005,1608,1583,1508,1461,1247,1176,1036$. HR-MS: $\mathrm{C}_{26} \mathrm{H}_{29} \mathrm{O}_{2}$ Calcd. 373.2168 (M+H); found: 373.2156.

$( \pm)-$ trans-4k

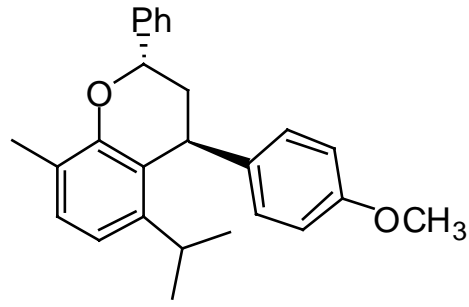

(士)-trans-5-Isopropyl-4-(4-methoxyphenyl)-8-methyl-2-phenylchroman. $\quad{ }^{1} \mathrm{H} \quad \mathrm{NMR}$ $\left(\mathrm{CDCl}_{3}\right)$ : 7.37-7.40 (m, 4H, PhCHO); 7.30-7.35 (m, $\left.1 \mathrm{H}, P h \mathrm{CHO}\right) ; 7.18$ (d, $1 \mathrm{H}, P h \mathrm{CH}_{3}, J$ $=7.8 \mathrm{~Hz}) ; 7.10\left(\mathrm{~d}, 2 \mathrm{H}, P h \mathrm{OCH}_{3}, J=8.7 \mathrm{~Hz}\right) ; 6.89\left(\mathrm{~d}, 2 \mathrm{H}, P h \mathrm{OCH}_{3}, J=8.7 \mathrm{~Hz}\right) ; 6.88(\mathrm{~d}$, $\left.1 \mathrm{H}, \mathrm{PhCH}_{3}, J=7.8 \mathrm{~Hz}\right) ; 4.97(\mathrm{dd}, 1 \mathrm{H}, \mathrm{PhCHO}, J=11.4$ and $2.8 \mathrm{~Hz}) ; 4.43(\mathrm{dd}, 1 \mathrm{H}$, $\mathrm{CH}_{3} \mathrm{OPhCH}, J=4.8$ and $\left.2.0 \mathrm{~Hz}\right) ; 3.84\left(\mathrm{~s}, 3 \mathrm{H}, \mathrm{OCH}_{3}\right) ; 2.83-2.89\left(\mathrm{~m}, 1 \mathrm{H}, \mathrm{CH}\left(\mathrm{CH}_{3}\right)_{2}\right)$; 2.34 (s, 3H, $\left.\mathrm{PhCH}_{3}\right) ; 2.32-2.36\left(\mathrm{~m}, 1 \mathrm{H}, \mathrm{CHCH}_{2}\right) ; 2.27-2.30\left(\mathrm{~m}, 1 \mathrm{H}, \mathrm{CHCH}_{2}\right) ; 1.20$ (d, $\left.3 \mathrm{H}, \mathrm{CH}\left(\mathrm{CH}_{3}\right)_{2}, J=6.8 \mathrm{~Hz}\right) ; 0.93\left(\mathrm{~d}, 3 \mathrm{H}, \mathrm{CH}\left(\mathrm{CH}_{3}\right)_{2}, J=6.8 \mathrm{~Hz}\right) .{ }^{13} \mathrm{C}\left\{{ }^{1} \mathrm{H}\right\} \mathrm{NMR} \delta 157.9$ (Quat.), 153.0 (Quat.), 146.1 (Quat.), 142.3 (Quat.), 138.2 (Quat.), 129.34 (Arom. CH), 129.26 (Arom. CH), 128.2 (Arom. CH), 127.4 (Arom. CH), 125.7 (Arom. CH), 123.3 (Quat.), 119.5 (Quat.), 116.9 (Arom. $\mathrm{CH}$ ), 113.6 (Arom. $\mathrm{CH}$ ), 71.8 (PhCHO), 55.2 $\left(\mathrm{OCH}_{3}\right) 39.4\left(\mathrm{ArCHCH}_{2}\right), 37.4(\mathrm{ArCHCH}), 28.1\left(\mathrm{CH}\left(\mathrm{CH}_{3}\right)_{2}\right), 24,8\left(\mathrm{CH}\left(\mathrm{CH}_{3}\right)_{2}\right), 23.0$ $\left(\mathrm{CH}\left(\mathrm{CH}_{3}\right)_{2}\right), 16.3\left(\mathrm{ArCH}_{3}\right) . V_{\max }(\mathrm{NaCl}) / \mathrm{cm}^{-1}: 3031,3004,1608,1581,1508,1461,1290$, 1247, 1228, 1203, 1053, 1034. HR-MS: $\mathrm{C}_{26} \mathrm{H}_{29} \mathrm{O}_{2}$ Calcd. $373.2168(\mathrm{M}+\mathrm{H})$; found: 373.2153 . 
Spectra of $\mathbf{4 k}$ from $\mathrm{PhSiH}_{3} / \mathrm{BF}_{3} \cdot \mathrm{Et}_{2} \mathrm{O}$ reduction

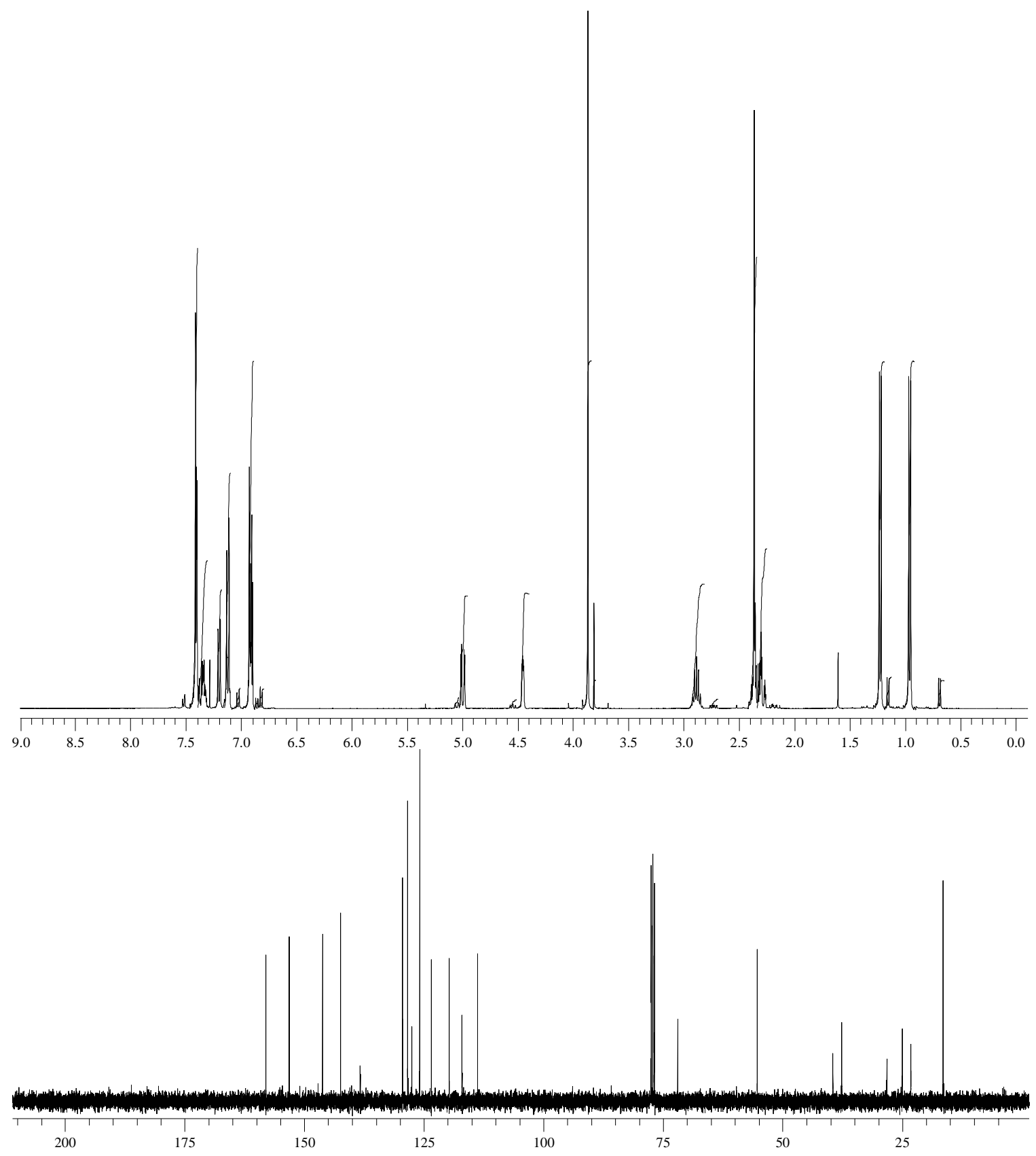




\section{Spectra of $\mathbf{4 k}$ from ${ }^{i} \mathrm{Pr}_{3} \mathrm{SiH} / \mathrm{BF}_{3} \cdot \mathrm{Et}_{2} \mathrm{O}$ reduction}

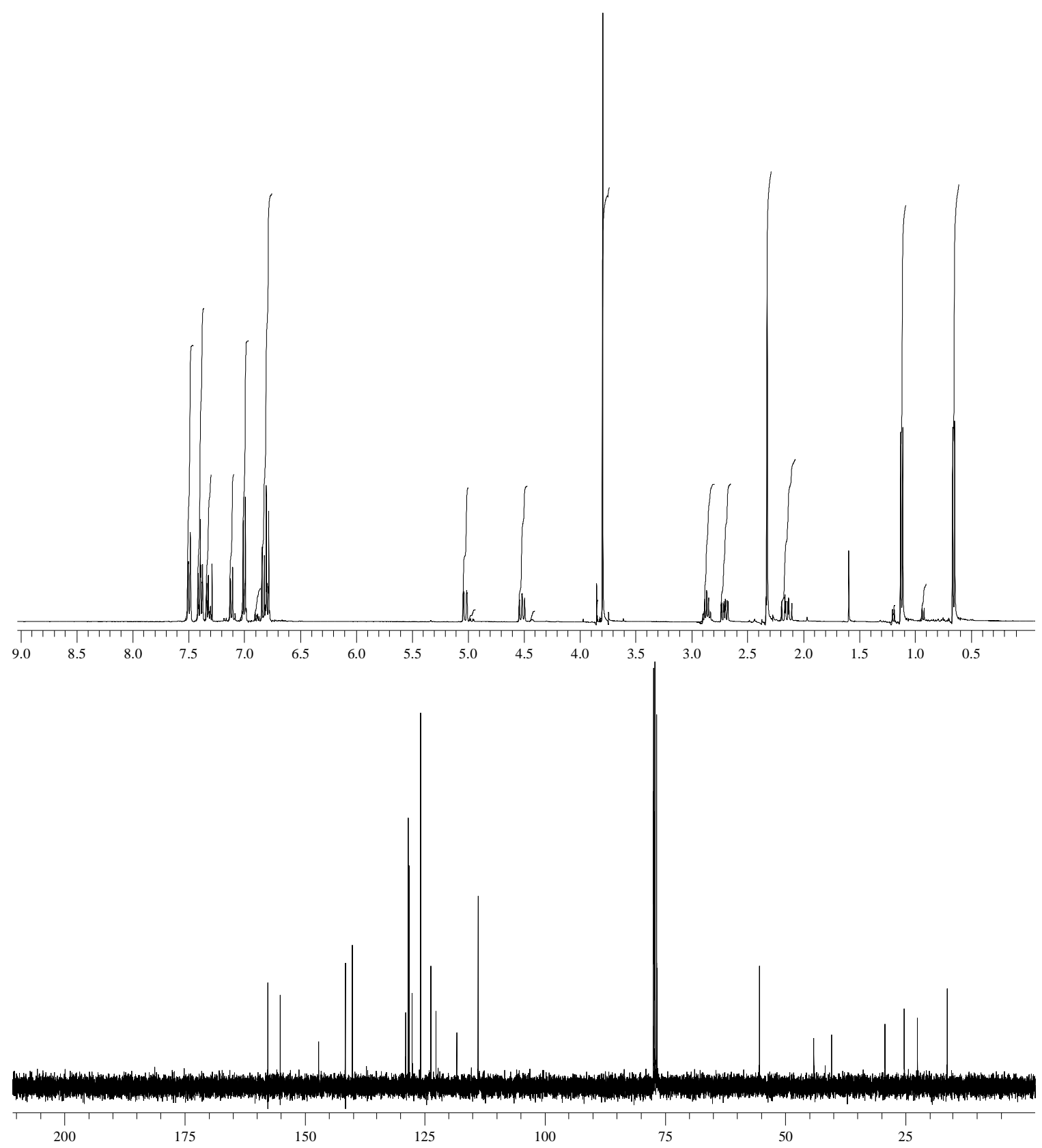


(士)-cis-41

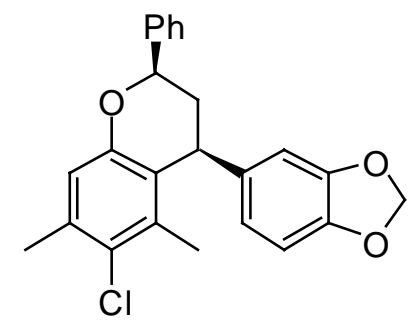

Column chromatography on silica gel $\left(\mathrm{DCM} /\right.$ hexane $\left.=1 / 1, \mathrm{R}_{\mathrm{f}}=0.4\right)$ to afford $4 \mathbf{l}$.

( \pm )-cis-4-(benzo[ $d][1,3]$ dioxol-5-yl)-6-chloro-5,7-dimethyl-2-phenylchroman. ${ }^{1} \mathrm{H}$ NMR $\left(\mathrm{CDCl}_{3}\right) \delta 7.41(\mathrm{~d}, 2 \mathrm{H}, P h \mathrm{CHO}, J=7.3 \mathrm{~Hz}) ; 7.36(\mathrm{t}, 2 \mathrm{H}, P h \mathrm{CHO}, J=7.3 \mathrm{~Hz}) ; 7.30(\mathrm{t}$, $1 \mathrm{H}, P h \mathrm{CHO}, J=7.3 \mathrm{~Hz}) ; 6.81(\mathrm{~s}, 1 \mathrm{H}, \mathrm{ClPh}) ; 6.69\left(\mathrm{~d}, 1 \mathrm{H}, P h \mathrm{OCH}_{2}, J=7.8 \mathrm{~Hz}\right) ; 6.55$ $\left(\mathrm{dd}, 1 \mathrm{H}, P h \mathrm{OCH}_{2}, J=8.1\right.$ and $\left.1.8 \mathrm{~Hz}\right) ; 6.47\left(\mathrm{~d}, 1 \mathrm{H}, P h \mathrm{OCH}_{2}, J=1.8 \mathrm{~Hz}\right) ; 5.90(\mathrm{~s}, 2 \mathrm{H}$, $\left.\mathrm{OCH}_{2} \mathrm{O}\right) ; 4.93(\mathrm{dd}, 1 \mathrm{H}, \mathrm{PhCHO}, J=11.4$ and $1.8 \mathrm{~Hz}) ; 4.31\left(\mathrm{dd}, 1 \mathrm{H}, \mathrm{OCH}_{2} \mathrm{OPhCH}, J=\right.$ 10.1 and $8.1 \mathrm{~Hz}) ; 2.57\left(\mathrm{ddd}, 1 \mathrm{H}, \mathrm{CHCH}_{2}, J=14.1,7.8\right.$ and $\left.1.8 \mathrm{~Hz}\right) ; 2.36\left(\mathrm{~s}, 3 \mathrm{H}, \mathrm{PhCH}_{3}\right)$; 2.08-2.17 (m, $1 \mathrm{H}, \mathrm{CHCH}_{2}$ ); 1.95 (s, 3H, $\left.\mathrm{PhCH}_{3}\right) .{ }^{13} \mathrm{C}\left\{{ }^{1} \mathrm{H}\right\}$ NMR $\delta 155.1$ (Quat.), 147.8 (Quat.), 145.7 (Quat.), 140.6 (Quat.), 140.2 (Quat.), 136.3 (Quat.), 135.7 (Quat.), 128.5 (Arom. CH), 127.95 (Arom. CH), 127.85 (Quat.), 126.0 (Arom. CH), 122.9 (Quat.), 120.2 (Arom. CH), 117.3 (Arom. $\mathrm{CH}$ ), 108.4 (Arom. $\mathrm{CH}$ ), 107.3 (Arom. $\mathrm{CH}$ ), 100.9 $\left(\mathrm{OCH}_{2} \mathrm{O}\right), 77.0(\mathrm{PhCHO}), 43.5\left(\mathrm{ArCHCH}_{2}\right), 42.0\left(\mathrm{ArCHCH}_{2}\right), 20.9\left(\mathrm{ArCH}_{3}\right), 18.5$ $\left(\mathrm{ArCH}_{3}\right) . v_{\max }(\mathrm{NaCl}) / \mathrm{cm}^{-1}: 1606,1564,1502,1485,1454,1440,1307,1267,1247,1232$, 1161, 1041. HR-MS: $\mathrm{C}_{24} \mathrm{H}_{21} \mathrm{ClO}_{3}$ Calcd. 392.1179 (M+); found: 392.1181 .

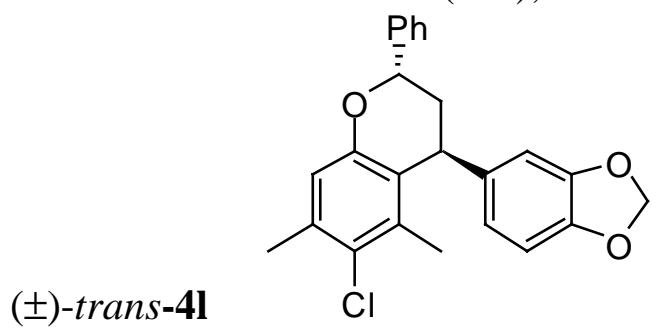

( \pm )-trans-4-(Benzo $[d][1,3]$ dioxol-5-yl)-6-chloro-5,7-dimethyl-2-phenylchroman (major). ${ }^{1} \mathrm{H}$ NMR $\left(\mathrm{CDCl}_{3}\right) \delta$ 7.29-7.37 (m, 5H, PhCHO); $6.83(\mathrm{~s}, 1 \mathrm{H}, \mathrm{ClPh}) ; 6.76(\mathrm{~d}, 1 \mathrm{H}$, $\left.P h \mathrm{OCH}_{2}, J=8.1 \mathrm{~Hz}\right) ; 6.64\left(\mathrm{~d}, 1 \mathrm{H}, P h \mathrm{OCH}_{2}, J=1.6 \mathrm{~Hz}\right) ; 6.55\left(\mathrm{dd}, 1 \mathrm{H}, P h \mathrm{OCH}_{2}, J=7.8\right.$ and $1.6 \mathrm{~Hz}) ; 5.96-5.97\left(\mathrm{~m}, 2 \mathrm{H}, \mathrm{OCH}_{2} \mathrm{O}\right) ; 4.89(\mathrm{dd}, 1 \mathrm{H}, \mathrm{PhCHO}, J=11.9$ and $2.0 \mathrm{~Hz})$; 4.23 (d, br, $\left.1 \mathrm{H}, \mathrm{OCH}_{2} \mathrm{OPhCH}, J=3.8 \mathrm{~Hz}\right) ; 2.39\left(\mathrm{~s}, 3 \mathrm{H}, \mathrm{PhCH}_{3}\right) ; 2.29-2.35(\mathrm{~m}, 1 \mathrm{H}$, $\left.\mathrm{CHCH}_{2}\right) ; 2.16-2.20\left(\mathrm{~m}, 1 \mathrm{H}, \mathrm{CHCH}_{2}\right) ; 2.11\left(\mathrm{~s}, 3 \mathrm{H}, \mathrm{PhCH}_{3}\right) \cdot{ }^{13} \mathrm{C}\left\{{ }^{1} \mathrm{H}\right\}$ NMR $\delta 153.5$ (Quat.), 147.8 (Quat.), 146.1 (Quat.), 141.2 (Quat.), 138.9 (Quat.), 136.0 (Quat.), 135.5 (Quat.), 128.4 (Arom. CH), 127.9 (Arom. CH), 126.8 (Quat.), 126.1 (Arom. $\mathrm{CH}$ ), 121.4 (Arom. CH), 120.1 (Quat.), 116.8 (Arom. CH), 108.6 (Arom. CH), 108.2 (Arom. CH), $101.0\left(\mathrm{OCH}_{2} \mathrm{O}\right), 72.2(\mathrm{PhCHO}), 39.3\left(\mathrm{ArCHCH}_{2}\right), 38.9\left(\mathrm{ArCHCH}_{2}\right), 20.9\left(\mathrm{ArCH}_{3}\right), 16.4$ $\left(\mathrm{ArCH}_{3}\right) . V_{\max }(\mathrm{NaCl}) / \mathrm{cm}^{-1}: 3034,3012,1606,1568,1502,1487,1456,1440,1305,1251$, 1232, 1168, 1101, 1041. HR-MS: $\mathrm{C}_{24} \mathrm{H}_{21} \mathrm{ClO}_{3}$ Calcd. 392.1179 (M+); found: 392.1176. 

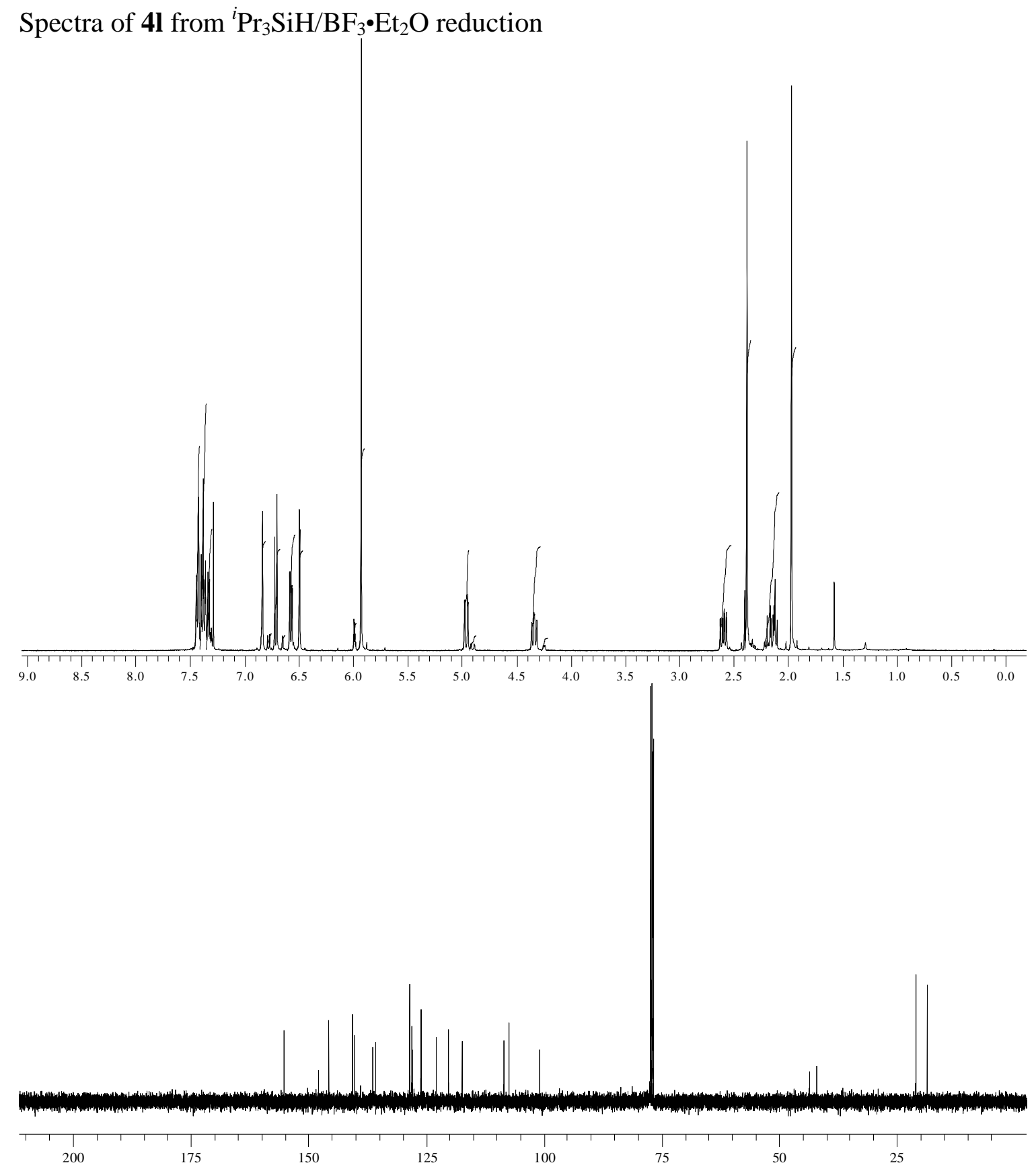
Spectra of $4 \mathbf{l}$ from $\mathrm{PhSiH}_{3} / \mathrm{BF}_{3} \cdot \mathrm{Et}_{2} \mathrm{O}$ reduction

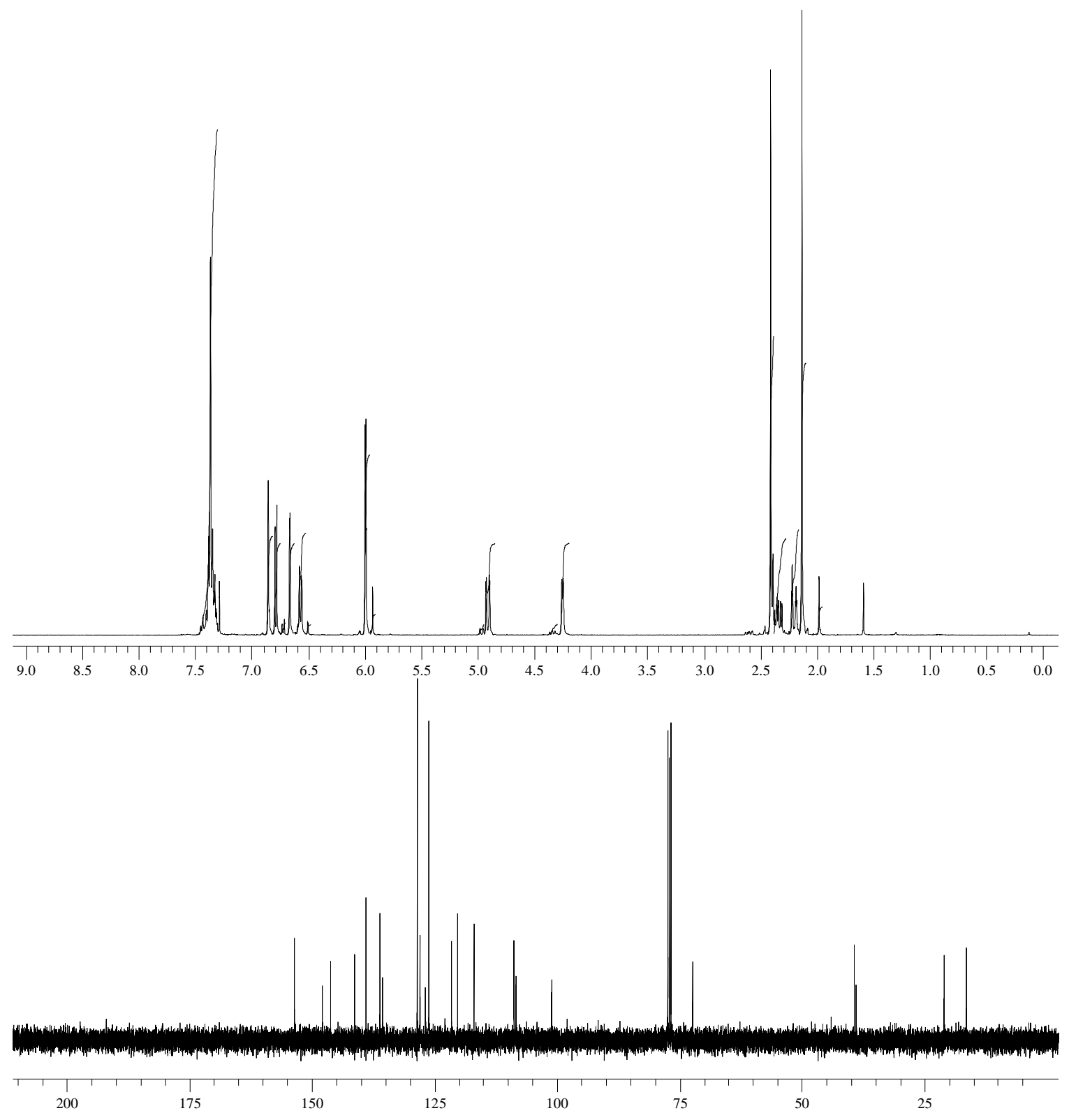

\section{Calculations: Introduction}

Figure S-1 shows two of the four complexes that we have studied. The first set of complexes has $\mathrm{R}=\mathrm{H}$ at the $\mathrm{C5}$ position of the chroman, and the second set has $\mathrm{R}=\mathrm{Me}$ at the C5 position (see Figure S-1). In either case, the phenyl ring on the C2 carbon can 
occupy the axial or the equatorial sites. Thus we have four geometries in total, and we have optimized these four complexes at different levels of theory to evaluate the difference in energy for the conformers with the phenyl in the axial and equatorial positions. That the geometries obtained were minima was confirmed with frequency calculations done for the geometries optimized at the B3LYP/6-31G* level.

Results

Figures S-1 and S-2 show the structures of the four complexes, optimized using density functional theory (DFT) with the B3LYP functional. The basis set employed for all the atoms was $6-31 \mathrm{G}^{*}$.

Table $\mathrm{S}-1$ shows the relative energies of the two conformers for the two cases $(R=$ $\mathrm{H}$ and $\mathrm{R}=\mathrm{Me}$ ) at different levels of theory. (Note: For the MP2 case, single point calculations were done with the geometries optimized at HF/3-21G; we have been unable to carry out calculations at the MP2/6-31G* due to memory and disk space limitations.) The best results should be considered the B3LYP/6-31G* calculations. For the $\mathrm{R}=\mathrm{H}$ case, the conformer with the phenyl in the equatorial position is favored by $0.40 \mathrm{kcal} / \mathrm{mol}$ at this highest level of theory (B3LYP/6-31G*), whereas, for the $R=\mathrm{Me}$ case, the conformer with the phenyl in the axial position is more stable by $3.23 \mathrm{kcal} / \mathrm{mol}$.

The energies reported here are the differences in electronic energies, i.e., $\Delta E$. We also calculated $\Delta \mathrm{H}$ for the $\mathrm{B} 3 \mathrm{LYP} / 6-31 \mathrm{G}^{*}$ case, and the values: $+0.42 \mathrm{kcal} / \mathrm{mol}$ and -3.20 $\mathrm{kcal} / \mathrm{mol}$ were found to be almost the same as the $\Delta E$ s obtained for this case.

We have also investigated the barrier to the flipping of one conformer to the other (at the B3LYP/6-31G* level). Figure S-3 shows the barriers obtained for $R=H$ and $R=M e$. The barrier increases from $3.78 \mathrm{kcal} / \mathrm{mol}$ to $4.85 \mathrm{kcal} / \mathrm{mol}$ upon the addition of the methyl group at the para position of the phenyl ring, thus indicating the steric effect of introducing the methyl group. Since the barriers are relatively low, significant interconversion between the two conformers can be expected even at $-78^{\circ} \mathrm{C}$. 

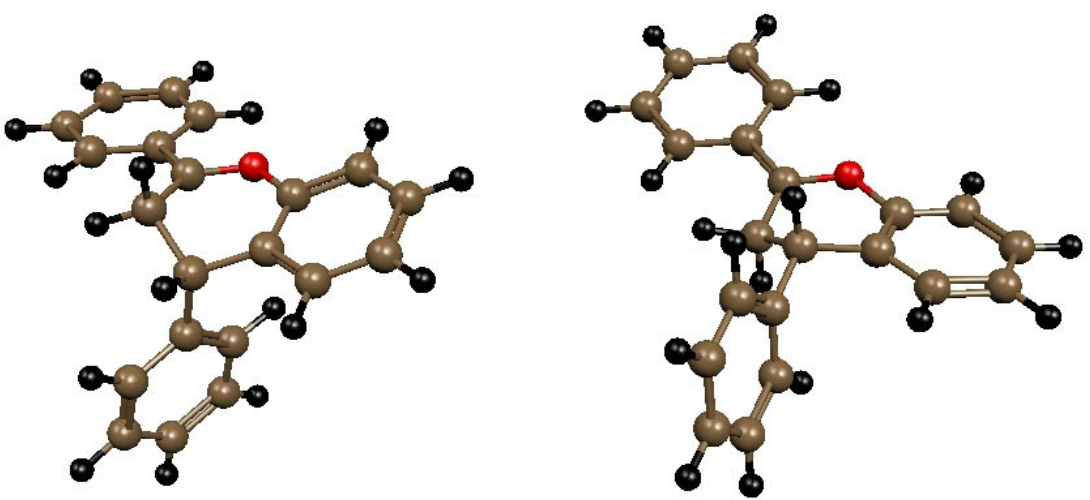

Figure S-1: DFT-optimized geometries of the structure having $\mathrm{R}=\mathrm{H}$, with the phenyl group in the axial (left) and equatorial (right) positions. 

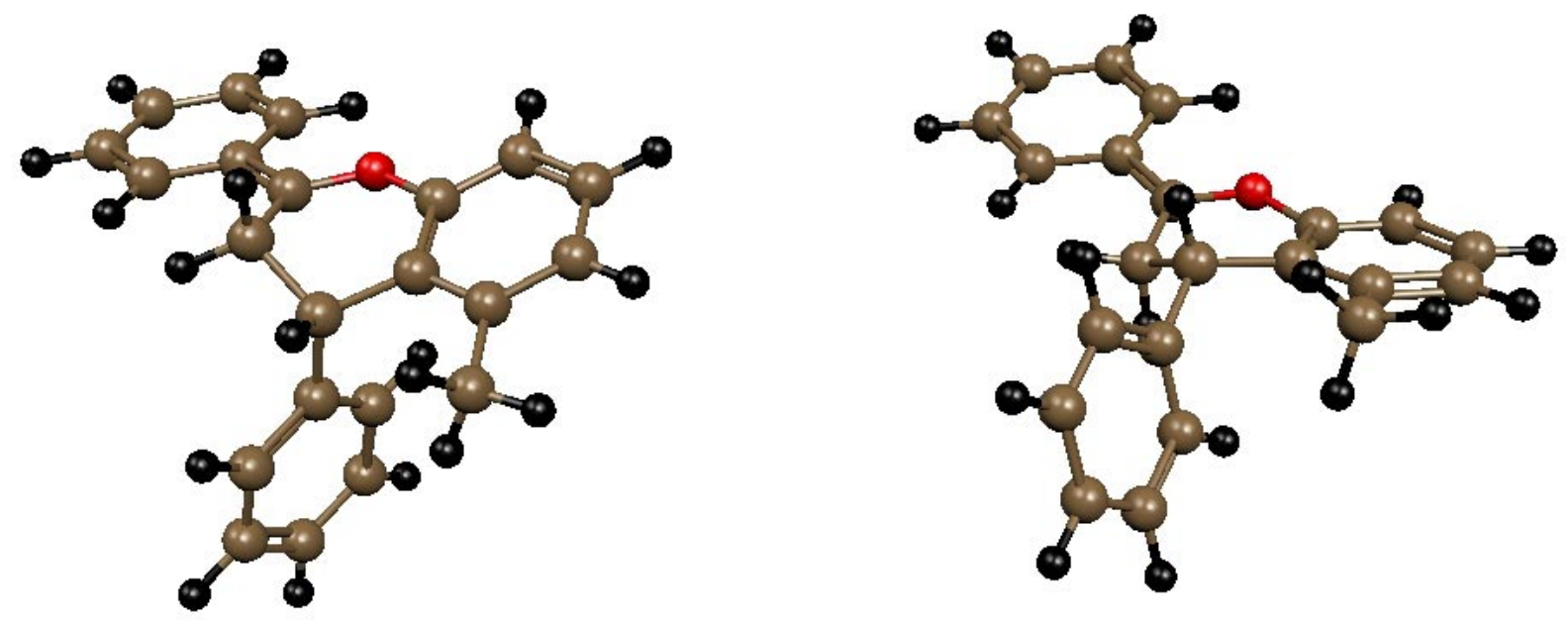

Figure S-2: DFT-optimized geometries of the structure having $\mathrm{R}=\mathrm{Me}$, with the phenyl group in the axial (left) and equatorial (right) positions.

Table S-1: The relative energies for different conformers at different levels of theory; the complex with the phenyl at the equatorial position is considered the reference point for the different cases.

\begin{tabular}{ccccc} 
Level & $\mathrm{R}=\mathrm{H}$, Phenyl Equat & $\mathrm{R}=\mathrm{H}$, Phenyl Axial & $\mathrm{R}=\mathrm{Me}$, Phenyl Equat & $\mathrm{R}=\mathrm{H}$, Phenyl Axial \\
\hline $\mathrm{kcal} / \mathrm{mol}$ & $\mathrm{kcal} / \mathrm{mol}$ & $\mathrm{kcal} / \mathrm{mol}$ & $\mathrm{kcal} / \mathrm{mol}$ \\
\hline $\mathrm{HF} / 3-21 \mathrm{G}$ & 0.0 & -0.42 & 0.0 & -4.58 \\
\hline $\mathrm{HF} / 6-31 \mathrm{G}^{*}$ & 0.0 & -0.98 & 0.0 & -3.91 \\
\hline B3LYP/6-31G* & 0.0 & +0.16 & 0.0 & -4.24 \\
\hline
\end{tabular}




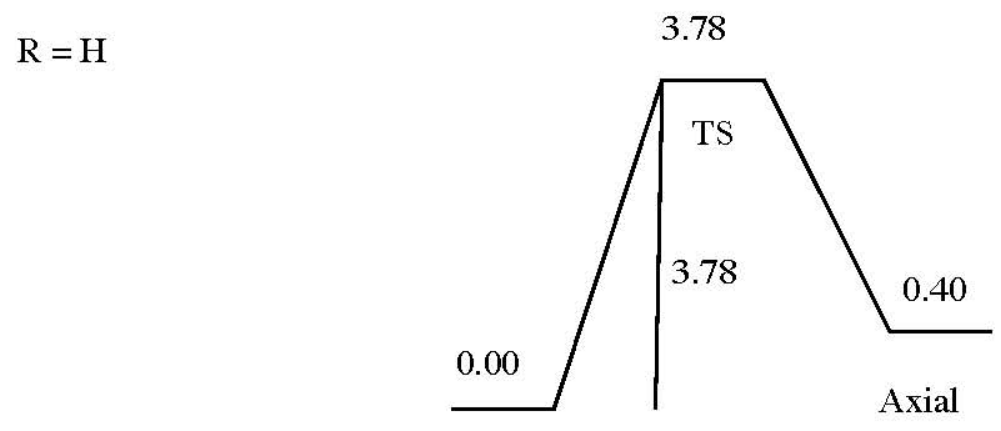

Equat

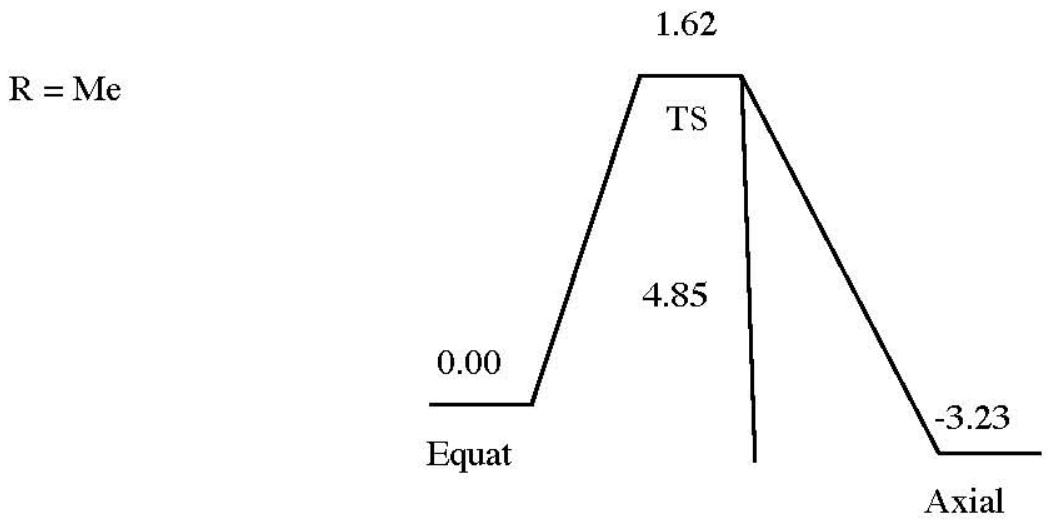

Figure S-3: The barriers to conformational equilibration for the cases $\mathrm{R}=\mathrm{H}$ and $\mathrm{R}=\mathrm{Me}$; all values in $\mathrm{kcal} / \mathrm{mol}$.

${ }^{1}$ Li, K.; Foresee, L. N.; Tunge, J. A. J. Org. Chem. 2005, 70, 2881-2883.

${ }^{2}$ Gaussian 98 (Revision A.7), M. J. Frisch, G. W. Trucks, H. B. Schlegel, G. E. Scuseria, M. A.Robb, J. R. Cheeseman, V. G. Zakrzewski, J. A. Montgomery, R. E. Stratmann, J. C. Burant, S. Dapprich, J. M. Millam, A. D. Daniels, K. N. Kudin, M. C. Strain, O. Farkas, J. Tomasi, V. Barone, M. Cossi, R. Cammi, B. Mennucci, C. Pomelli, C. Adamo, S. Clifford, J. Ochterski, G. A. Petersson, P. Y. Ayala, Q. Cui, K. Morokuma, D. K. Malick, A. D. Rabuck, K. Raghavachari, J. B. Foresman, J. Cioslowski, J. V. Ortiz, B. B. Stefanov, G. Liu, A. Liashenko, P. Piskorz, I. 
Komaromi, R. Gomperts, R. L. Martin, D. J. Fox, T. Keith, M. A. Al-Laham, C. Y. Peng, A. Nanayakkara, C. Gonzalez, M. Challacombe, P. M. W. Gill, B. G. Johnson, W. Chen, M. W. Wong, J. L. Andres, M. Head-Gordon, E. S. Replogle and J. A. Pople, Gaussian, Inc., Pittsburgh PA, 1998. 